\title{
The Equation of State of Hot, Dense Matter and Neutron Stars
}

\author{
James M. Lattimer ${ }^{2}$ \\ Department of Physics 85 Astronomy, Stony Brook University, Stony Brook, NY 11733, \\ USA \\ Madappa Prakash ${ }^{1}$ \\ Department of Physics and Astronomy, Ohio University, Athens, OH 45701, USA
}

\begin{abstract}
Recent developments in the theory of pure neutron matter and experiments concerning the symmetry energy of nuclear matter, coupled with recent measurements of high-mass neutron stars, now allow for relatively tight constraints on the equation of state of dense matter. We review how these constraints are formulated and describe the implications they have for neutron stars and corecollapse supernovae. We also examine thermal properties of dense matter, which are important for supernovae and neutron star mergers, but which cannot be nearly as well constrained at this time by experiment. In addition, we consider the role of the equation of state in medium-energy heavy-ion collisions.

Keywords: Neutron stars; Dense matter in equilibrium; Thermal effects;

Heavy-ions; Dense matter off-equilibrium; Equation of state of dense matter
\end{abstract}

\footnotetext{
${ }^{1}$ prakash@ohio.edu

2 james.lattimer@stonybrook.edu

Preprint submitted to Physics Reports
}

December 19, 2015 


\section{Contents}

\begin{tabular}{|lll}
\hline 1 & INTRODUCTION & 4
\end{tabular}

2 THE EOS AND NEUTRON STAR STRUCTURE 9

2.1 Results from Dimensional Analysis . . . . . . . . . . . . . . . 11

5 2.2 The Maximally-Compact EOS: The Roles of Causality and the

Maximum Mass . . . . . . . . . . . . . . . . . . . . . . . . . . . . 14

2.3 The Neutron Star Crust and the Low-Density EOS . . . . . . . . 17

2.4 The Intermediate EOS and Pure Neutron Matter . . . . . . . . . 19

3 Nuclear Structure and the Nuclear Symmetry Energy 22

10

3.1 Hydrodynamic Model of the Nuclear Symmetry Energy $\ldots . . .22$

3.1 .1 The isoscalar density $n \ldots \ldots \ldots \ldots$

3.1 .2 The isovector density $\alpha \ldots \ldots \ldots \ldots$

$3.1 .3 \quad$ Nuclear structure . . . . . . . . . . . . . . . . . . . 26

$3.1 .4 \quad$ Solutions for arbitrary $v_{\text {sym }} \ldots \ldots \ldots \ldots \ldots 28$

15

$3.1 .5 \quad$ Predicted experimental correlations . . . . . . . . . 30

3.2 Symmetry Parameter Constraints from Experiment . . . . . . . . 33

$4 \quad$ The High-Density EOS and Mass and Radius Constraints 35

4.1 A First Model: Fixed Boundaries . . . . . . . . . . . . . . . . . . 36

$4.2 \quad$ A Second Model: Phase Transitions . . . . . . . . . . . . . . . . 41

205 Summary of Observational Constraints 43

5.1 Photospheric Radius Expansion Bursts . . . . . . . . . . . . . . 43

5.2 Quiescent Low-Mass X-Ray Binaries . . . . . . . . . . . . 46

5.3 Bayesian Analyses of Observations with Theoretical Priors. . . . 48

5.4 Other Observations . . . . . . . . . . . . . . . . . . . . . . . . . 49

25

5.4 .1 Moments of Inertia . . . . . . . . . . . . . . . . . . . . 49

5.4 .2 Pulse-profile observations . . . . . . . . . . . . . 52

$5.4 .3 \quad$ Gravitational wave observations. . . . . . . . . . . . . 53

5.4 .4 Rotation Periods . . . . . . . . . . . . . . . 55 
$5.4 .5 \quad$ Binding Energies $\ldots \ldots \ldots \ldots$

30 6 THERMAL EFFECTS IN BULK MATTER 57

6.1 Non-degenerate Limit . . . . . . . . . . . . . . . . . . . 57

6.2 Degenerate Limit . . . . . . . . . . . . . . . . . . 58

$6.2 .1 \quad$ Non-relativistic models. . . . . . . . . . . . . . . . . . . 59

6.2 .2 Relativistic models . . . . . . . . . . . . . . . . . 60

$35 \quad 6.3$ Illustrative Results $\ldots \ldots \ldots \ldots \ldots$. . . . . . . . . . . 61

6.3 .1 Non-relativistic models. . . . . . . . . . . . . . . . . 61

6.3 .2 Relativistic field-theoretical models . . . . . . . . . . . 66

6.4 General Comments . . . . . . . . . . . . . . . . . . 70

\begin{tabular}{|lll}
\hline & NON-THERMAL EFFECTS IN BULK MATTER & 71
\end{tabular}

$\begin{array}{lll}40 & \text { CONCLUSIONS } & 75\end{array}$ 


\section{INTRODUCTION}

Gerry was long interested in the equation of state (EOS) of dense matter and supernovae, and formulated many ideas concerning the mechanism underlying core-collapse supernovae. Gerry Brown was not one who would let a crisis or controversy in nuclear physics pass him by. Whether the dense matter EOS was soft or stiff was a debate he plunged into with great gusto. To enable a core-collapse supernova explode through the prompt-shock and rebound mechanism, Gerry argued [1] that the EOS at nuclear densities had to be soft with an incompressibility parameter, $K_{s}$, of isospin symmetric nuclear matter much smaller $(\sim 110 \mathrm{MeV})$ than $220 \pm 30 \mathrm{MeV}$ inferred from the analysis of giant monopole resonances by Blaizot et al. [2. Thinking today supports the notion that neutrinos and multi-dimensional effects are required to ensure successful explosions. While he also famously argued for a relatively soft nuclear EOS to explain why no neutron star has yet been detected in the remnant of SN 1987A, observations of pulsars today indicate that the maximum mass is quite large [3, 4. In his later years, author JML would try to provoke him (in retaliation for phone calls, during Notre Dame football games, purporting to discuss physics) with tales of ever larger neutron star mass measurements. Ironically, it now appears that uncertainties in the dense matter EOS have little to do with uncertainties in the core-collapse supernova mechanism.

Nevertheless, he does not appear to have been completely wrong about softness in the EOS. While experiments [5, 6, 7] indicate that symmetric matter has a larger incompressibility than he favored, pure neutron matter, which is much closer to neutron star matter than is symmetric matter, seems to be relatively soft near the nuclear saturation density. Calculations of the properties of pure neutron matter, as well as experimental results concerning the symmetry properties of dense matter gleaned from experiments measuring binding energies, neutron skin thicknesses and giant resonances, support this perspective. But to attain a large maximum mass, the EOS at densities beyond twice the nuclear 70 saturation density must become very stiff. Gerry would have been fascinated 
with this development.

Gerry was also intimately involved in great debates in the late 1970s concerning thermal effects in dense matter, and strongly argued, with Hans Bethe, that the importance of excited states in nuclei had been underappreciated. His75 torical comments concerning his work, which culminated in the "BBAL" [8] paper, are contained in articles appearing in the recent Nuclear Physics Memorial Volume [9, 10. In this case, of course, he was correct. The role of thermal effects has taken on new emphasis with the realization that high entropies and temperatures exist in hypermassive neutron stars, the metastable, differentially rotating hot configurations which are the aftermath of some neutron star mergers. Their lifetimes before gravitational collapse to black holes ensues crucially depend on the specific heat of the hot matter as well as on neutrino emissivities which determine cooling rates and timescales for dissipation of differential rotation.

In the 1980's data about collective flow from 0.5-2 GeV per nucleon heavy-ion collisions at the Bevalac became available. Initial theoretical analyses indicated that the EOS at near-nuclear and supra-nuclear densities was very stiff with an incompressibility parameter close to $400 \mathrm{MeV}$. In many works, even larger values were predicted. Gerry and author MP struggled a lot to reconcile such large values with the much lower values suggested by the analyses of the giant resonances data and theoretical calculations of the EOS. MP's first paper with Gerry [11] was a tortuous experience insofar as none of the authors was convinced about the resolution of the problem on hand. We argued fiercely about how the paper was to be written. After many drafts, Gerry relegated text of MP's detailed calculations to the appendices, and replaced the main text with many conflicting ideas. The paper took over a year to get published with many revisions after the referee's comments. One of those ideas, that the momentum dependent interactions could be at the root of the solution, turned out to be right and has stood the test of time. As always, Gerry was generous to competitors (behind their backs, of course); the note added in proof acknowledges preprints by Aichelin et al., [12] and Gale et el., [13] which were submited after 
the completion of our work. These works had similar ideas and calculations moving in the right direction, although they were incomplete.

Not being satisfied with what had transpired, MP suggested to Gerry a

need for the momentum dependence to saturate at high momenta to describe heavy-ion data. In addition, the cold EOS implied by such a mean field is also 
consistent with the recently discovered $2 \mathrm{M}_{\odot}$ neutron star [22, 23. Much of the credit for the resolution of the thorny problem mentioned above belongs to Gerry who gave the team he built much impetus.

Gerry's penchant for effective masses is well reflected in his prescient paper "Effective Mass in Nuclei" written with Gunn and Gould in 1963 [24]. The abstract of this paper stated "Calculations in finite nuclei indicate that in the region of bound particles $\approx-8$ to $0 \mathrm{MeV}$ the velocity dependence of the shell model is, if present, opposite to that usually assumed. This can be expressed by saying that the ratio of the effective mass to the real mass is equal to or greater than unity in this energy range." The last sentence of the paper read "It seems strange to us that people making calculations in nuclear matter do not worry about this point, however, since it remains in direct conflict with our conclusions, unless understood as a specific effect of the finiteness." This was Gerry at his provocative best in print! Effective masses are central to the delineation of thermal effects and are further discussed in Sec. 6.

Gerry's protective attitude toward his wards was unmatched. When Gerry became seriously ill, he forwarded his last graduate student, Constantinos Constantinou, to Ohio University. The authors co-advised Constantinos, who completed his Ph.D. degree from Stony Brook in 2013. Constantinos (now a post-doc at Juelich with Ulf-Meissner, another of Gerry's wards) has worked on thermal effects in dense matter relevant to the astrophysics of compact objects, a subject that was close to Gerry's heart. This article contains a brief review of Constantinos's work [23, 25, 26, which would have pleased Gerry immensely especially as Landau's Fermi Liquid theory (another subject close to Gerry's heart) was put to good use.

This review will highlight important recent developments in our understanding of the EOS of dense, hot matter. In section 2, the basics of neutron star structure are developed, and constraints for neutron star radii based on general relativity, causality and pulsar-timing measurements of neutron star masses are outlined. A lot hinges upon the behavior of the nuclear symmetry energy. It is now realized that the radius of typical star, usually taken to be $1.4-1.5 M_{\odot}$, is 
closely connected to the pressure of neutron star matter near the nuclear saturation density $n_{s} \sim 0.16 \mathrm{fm}^{-3}$. Neutron star matter near $n_{s}$ has a very small proton fraction, so that it is nearly the same as pure neutron matter, which has been the focus of activity in recent years. And given that the pressure of neutron star matter can be computed from symmetric nuclear matter with knowledge of the nuclear symmetry energy, there is also a direct connection between neutron star structure and experiments that probe the symmetry energy, namely those measuring binding energies, neutron skin thicknesses and giant resonances. The evidence from nuclear experiments supporting symmetry energy results deduced from neutron matter studies is explored in section 3.2 . The refined constraints developed along these two parallel tracks have definite implications for neutron 175 star structure, when they are coupled with the existence of the hadronic neutron star crust. By parameterizing the high-density EOS, these constraints can be quantified, which is the subject of section 4.

All neutron stars emit photons releasing their thermal energy. If they were blackbody emitters, measurements of their fluxes, temperatures and distances would suffice to measure neutron star radii, a subject discussed in section 5. Even though neutron star atmospheres modify their spectra so that they are not true blackbodies, it is apparent that astronomical observations can closely connect to neutron star radii. Although measurements of neutron star radii from observations are not yet accurate enough to compete with inferences obtained from nuclear experiment and theory, several proposals for improvements in this direction are discussed. In section 6, thermal effects in dense matter are reviewed. Uncertainties stemming from the lack of knowledge of effective masses at high densities, as well as model dependences arising from the nature of the nuclear interaction model, are discussed. In section 7, non-thermal effects of importance to heavy-ion collisions are discussed. Emphasis is placed on the role of the momentum dependence in the single particle potential. In section 8 we summarize the main points of this review. 


\section{THE EOS AND NEUTRON STAR STRUCTURE}

The EOS of finite temperature matter is an essential ingredient in the mod-

195

used to gauge the ambient physical conditions. Examples of EOS tabulations can be found, e.g., in Refs. [27, 28, 29, 30, 31, 32, 33, 34, 35, 36.

\begin{tabular}{cccc}
\hline & $\begin{array}{c}\text { Core-collapse } \\
\text { supernovae }\end{array}$ & $\begin{array}{c}\text { Proto-neutron } \\
\text { stars }\end{array}$ & $\begin{array}{c}\text { Mergers of compact } \\
\text { binary stars }\end{array}$ \\
\hline$n / n_{s}$ & $10^{-8}-10$ & $10^{-8}-10$ & $10^{-8}-10$ \\
$T(\mathrm{MeV})$ & $0-30$ & $0-50$ & $0-100$ \\
$Y_{e}$ & $0.35-0.45$ & $0.01-0.3$ & $0.01-0.6$ \\
$S\left(k_{B}\right)$ & $0.5-10$ & $0-10$ & $0-100$ \\
\hline
\end{tabular}

Table 1: Ranges of baryon number density $n$, temperature $T$, net electron fraction $Y_{e}=n_{e} / n$, and entropy per baryon $S$ encountered in the indicated astrophysical phenomena.

At relatively low temperatures, matter below $\sim 0.1 \mathrm{fm}^{-3}$ comprises an inhomogeneous mixture of unbound nucleons (mostly neutrons), light nuclear clusters ( $\alpha$ particles, deuterons, etc.), heavy nuclei, and electrons. At densities less than the neutron-drip density, about $0.0016 n_{s}$, only heavy nuclei and electrons exist. At densities beyond $0.01 n_{s}$, competition between surface and Coulomb energies deforms nuclei, resulting in the evenutal formation of pasta-like configurations around $n \simeq 0.1 n_{s}$. At $n \sim 0.5 n_{s}$, a phase transition from inhomo210 geneous to homogeneous matter occurs. In the inhomogeneous phase above a certain temperature the relative proportion of nuclei dwindles, and a state of homogeneous matter forms. At supra-nuclear densities, probably beyond $2 n_{s}$, 
Bose condensates, strangeness-bearing baryons, mesons, and quarks may also be present. These phases are illustrated schematically in Fig. 1
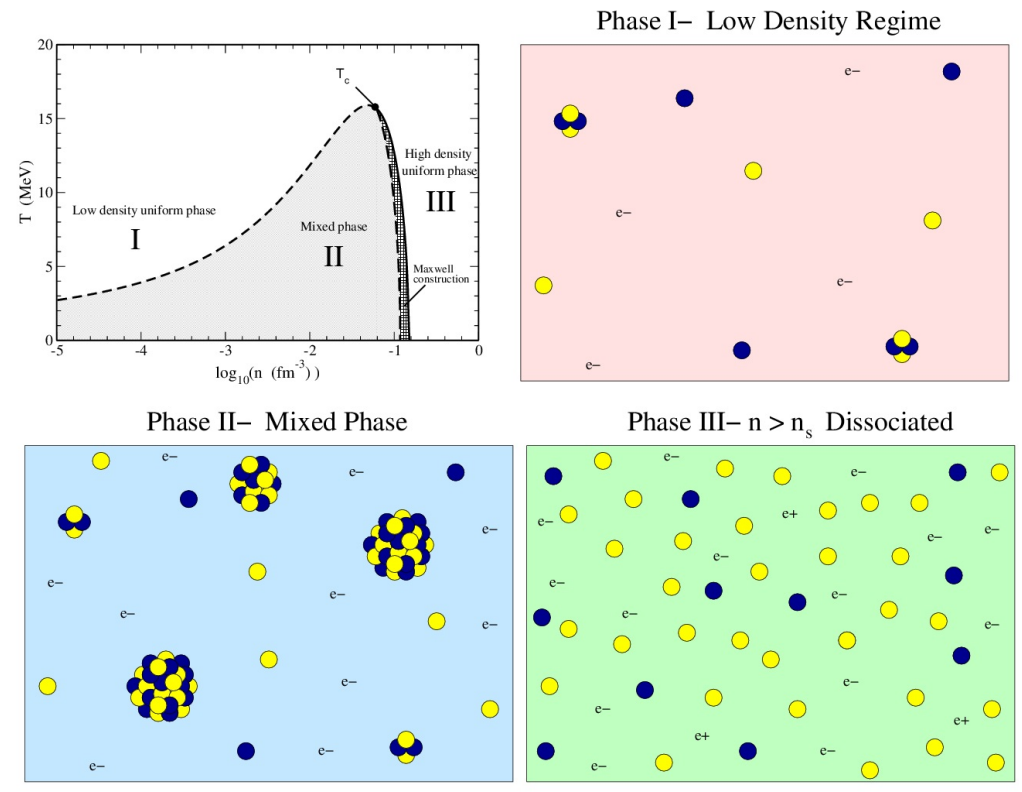

Figure 1: Phases of sub-nuclear matter. The upper left figure shows the boundary separating the three phases illustrated in the other pictures. Figure from Ref. [37.

The global properties of neutron stars mostly depend on the EOS of matter above the saturation density. Two of the outstanding problems in nuclear astrophysics concern the maximum mass for neutron stars and the value of the radius of a typical $1.4 M_{\odot}$ star. These problems are coupled: it is shown below that an observed lower limit for the maximum mass implies a minimum value for this radius. Recent measurements of neutron star masses by pulsar timing have led to the discovery of stars with masses much larger than seemed likely a decade ago, so that this constraint is no longer academic but practical.

Neutron stars are so compact that general relativity (GR) is essential in determining their structure. Nevertheless, some useful insights can be obtained 
by considering the spherical Newtonian structure equations:

$$
\frac{d p}{d r}=-\frac{G m \rho}{r^{2}}, \quad \frac{d m}{d r}=4 \pi \rho r^{2},
$$

where $\rho$ is the mass density and $p$ is the pressure. Above, $m$ represents the mass interior to the radius $r$. It is necessary to supply the $\operatorname{EOS} p(\rho)$ to solve either the limiting mass or radius. These features are apparent in the $M-R$ curves displayed in Fig. 2 ,

The close connection between neutron star radii and $K$ was exploited by Lattimer \& Prakash [38] who found phenomenological correlations between the 


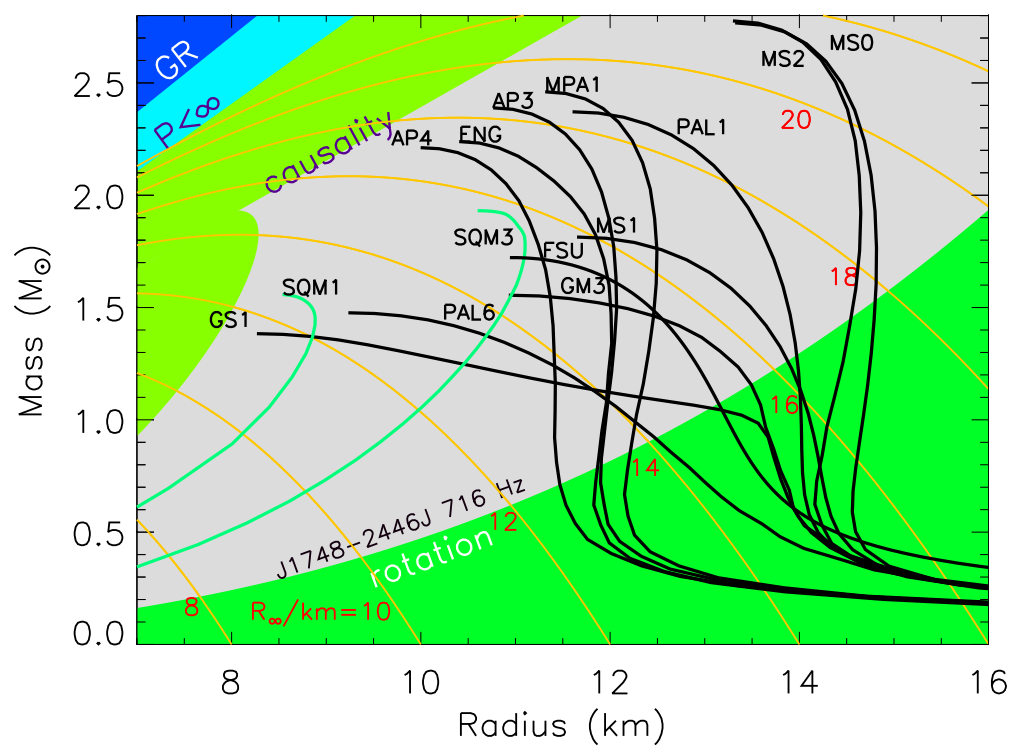

Figure 2: Mass-radius curves for a variety of popular EOSs (see Ref. [38 for descriptions). The green shaded region in the upper left is causally-excluded; the green shaded region in the lower-right is excluded by the most rapidly spinning pulsar. Black curves are hadronic EOSs; green curves are for strange quark matter configurations. Lines of fixed $R_{\infty}=R / \sqrt{1-2 \beta}$ are indicated as orange curves.

pressure of neutron star matter at selected densities, $p_{\beta}(n)$ in units of $\mathrm{MeV}$ $\mathrm{fm}^{-3}$, and the radius of a typical $1.4 M_{\odot}$ star ${ }^{3}$

$$
R_{1.4}=(9.52 \pm 0.49)\left[p_{\beta}\left(n_{s}\right)\right]^{1 / 4} \mathrm{~km} ; R_{1.4}=(5.68 \pm 0.14)\left[p_{\beta}\left(2 n_{s}\right)\right]^{1 / 4} \mathrm{~km}
$$

The spread of $R_{1.4}$ observed in Fig. (2), between 9 and $15 \mathrm{~km}$, reflects uncertainties in the pressure. Fig. (2) shows, however, that the spread of $R_{1.4}$ becomes smaller $(11-15 \mathrm{~km})$ if EOSs are required to support masses of $2.0 M_{\odot}$ rather than $1.4 M_{\odot}$. We will show that the radius uncertainty is further reduced due to additional theoretical and experimental constraints.

The introduction of GR results in strong gravity for large compactness (small

\footnotetext{
${ }^{3}$ This displayed correlations, and one standard deviation errors, are revisions [39] that incorporate only those EOSs that can support observed neutron star masses.
} 
values of $\beta$ ). The structure equations become

$$
\frac{d P}{d r}=-\frac{G\left(m c^{2}+4 \pi r^{3} p\right)(\epsilon+p)}{r c^{4}\left(r-2 G m / c^{2}\right)} ; \quad \frac{d m}{d r}=4 \pi \frac{\epsilon}{c^{2}} r^{2},
$$

where $\epsilon$ is the total mass-energy density, defined by $\epsilon=n\left(m_{b} c^{2}+E\right)$, with $m_{b}$ the baryon mass and $E$ the internal energy per baryon. In the GR case, there exists a maximum mass irrespective of whether or not causality is violated. Imposition of causality, of course, lowers the maximum mass.

An important distinction between GR and the Newtonian case is the appearance of the mass density $\rho=n m_{b} c^{2}$ in the latter instead of the total energy density $\epsilon$. As a result, only if $\gamma=d \ln p / d \ln \rho \leq 4 / 3$ in the high-density limit will a maximum mass exist in the Newtonian case ${ }^{4}$. Enforcing causality in the Newtonian sense therefore results in a maximum mass, since $\gamma \leq 1$ in this case in the high-density limit. For neutron stars this is an artificial situation, however, since enforcing causality in the relativistic sense $d p / d \epsilon=1$ implies that $d \ln p / d \ln \rho=\gamma=2$ for any realistic EOS at high densities, and no maximum mass will therefore exist. The existence of the Chandrasekhar mass for a Newtonian white dwarf is a consequence of restricting densities to values below where baryonic pressure becomes substantial so that $\gamma=4 / 3$.

A simple demonstration of the GR situation occurs in the incompressible limit, where the density in the star is constant irrespective of the pressure. This is effectively the case $\gamma \rightarrow \infty$, and Eq. (1) shows that $M \propto K^{0} R^{3}$ in the Newtonian limit and there is no maximum mass. Since the central density in the GR case is $\epsilon_{c}=3 M c^{2} /\left(4 \pi R^{3}\right)$, the explicit solution of Eq. (4) is

$$
p(r)=\epsilon_{c}\left[\frac{\sqrt{1-2 \beta(r / R)^{2}}-\sqrt{1-2 \beta}}{3 \sqrt{1-2 \beta}-\sqrt{1-2 \beta(r / R)^{2}}}\right] .
$$

The denominator vanishes at the star's center for $\beta=4 / 9$, which represents the maximum possible compactness 5 . These limits to $\beta$ hold for any EOS, not

\footnotetext{
${ }^{4}$ Eq. 22 shows that $M \propto \rho_{c}^{(3 \gamma-4)} / 2$, so if $\gamma>4 / 3, M$ increases without bound with increasing central density $\rho_{c}$.

${ }^{5}$ Note the condition $p_{c} \leq \epsilon_{c}$, required by causality, gives an even more restrictive limit $\beta \leq 3 / 8$.
} 
just the incompressible case (see Sec. 11.6 in Ref. [40]). The $\beta=4 / 9$ limit is indicated together with the black hole limit $\beta=1 / 2$ in Fig. 2

The denominator term $r-2 G m / c^{2}$ has a large effect in modifying the $M-R$ curves, but it is not the reason a maximum mass exists. If it is ignored, the incompressible fluid has the explicit solution

$$
p(r)=\epsilon_{c}\left(\frac{\exp \left[2 \beta\left(1-r^{2} / R^{2}\right) / 3\right]-1}{3-\exp \left[2 \beta\left(1-r^{2} / R^{2}\right) / 3\right]}\right) .
$$

The denominator vanishes at the center for $\beta=(3 / 2) \ln 3 \simeq 1.65$, so the maximum compactness is about 3.7 times larger than when including this term 6 Mass

An incompressible fluid has an infinite sound speed squared, $c_{s}^{2} / c^{2}=s=$ $\partial p / \partial \epsilon$, and is therefore unrealistic. Imposition of causality, namely that $s \leq 1$, leads to a larger minimum radius, $R \gtrsim 2.823 G M / c^{2}$ [41]. Koranda, Stergioulas $\&$ Friedman [42] conjectured that the most compact configurations are produced when the low-density EOS is very soft (i.e., low pressure) and the high-density EOS is very stiff. The maximally-compact EOS is therefore

$$
p=\epsilon-\epsilon_{0} \quad \text { when } \quad \epsilon \geq \epsilon_{0} ; \quad p=0 \quad \text { when } \quad \epsilon<\epsilon_{0} .
$$

In this case, the structure equations can be cast into a scale-free form by substitution of the variables

$$
w=\epsilon / \epsilon_{0}, \quad x=r \sqrt{G \epsilon_{0}} / c^{2}, \quad y=m \sqrt{G^{3} \epsilon_{0}} / c^{4} .
$$

There is a single parameter $\epsilon_{0}$, aside from the constants $G$ and $c$, and the dimensionless TOV equations become

$$
\frac{d w}{d x}=-\frac{\left(y+4 \pi x^{3} w\right)(2 w-1)}{x(x-2 y)}, \quad \frac{d y}{d x}=4 \pi x^{2} w,
$$

with boundary values $y=0$ and $d w / d x=0$ when $x=0$. The surface is where the pressure vanishes, or $w=1$. At the surface, $x$ and $y$ attain the

${ }^{6}$ With the condition $p_{c} \leq \epsilon_{c}$, the more restrictive limit $\beta=(3 / 2) \ln 2 \simeq 1.04$ is obtained. 
values $x_{1}=x(w=1)$ and $y_{1}=y(w=1)$, respectively. The central value of $w, w_{0}$, defines a family of solutions. The maximally compact solution occurs when $y_{1}$ is maximized. This occurs when $w_{0, c}=3.034$, for which $x_{1, c}=0.2405$ and $y_{1, c}=0.08522$ [43. This gives the causally limited compactness as $\beta_{c}=$ $y_{1, c} / x_{1, c}=0.3543=1 / 2.823$, which is independent of $\epsilon_{0}$.

The maximum neutron star mass is scaled by $\epsilon_{0}$ :

$$
M_{\max }=\frac{y_{1, c} c^{4}}{\sqrt{G^{3} \epsilon_{0}}} \simeq 4.09 \sqrt{\frac{\epsilon_{s}}{\epsilon_{0}}} M_{\odot}
$$

where $\epsilon_{s} \simeq 150 \mathrm{MeV} \mathrm{fm}^{-3}$ is the energy density at $n_{s}$. This is essentially the result first found by Rhoades \& Ruffini 44. Since nuclear structure shows that a phase transition does not occur below $n_{s}$, the maximum neutron star mass must be less than about $4.1 M_{\odot}$. In addition, the largest accurately measured neutron star mass, $M_{\max } \simeq 1.97 M_{\odot}$ [3, 4], which must be less than the true maximum mass, gives an upper limit to $\epsilon_{0}, \epsilon_{0, \max }$. Upper limits to the central energy density and pressure then follow:

$$
\begin{aligned}
& \epsilon_{c, \text { max }}=w_{0, c} \epsilon_{0, \text { max }} \simeq 50.8 \epsilon_{s}\left(\frac{M_{\odot}}{M_{\max }}\right)^{2}, \\
& p_{c, \max }=\left(w_{0, c}-1\right) \epsilon_{0, \max } \simeq 34.1 \epsilon_{s}\left(\frac{M_{\odot}}{M_{\max }}\right)^{2} .
\end{aligned}
$$

The discovery of a star with $M>1.97 M_{\odot}$ would decrease these limits.

Another result immediately follows: the minimum radius for a neutron star of mass $M \leq M_{\max }$ is determined by the coupled equations [45]

$$
M=M_{\max } \frac{y_{c}}{y_{c, 1}}, \quad R=\frac{G M_{\max }}{c^{2}} \frac{x_{c}}{y_{c, 1}}=\frac{G M}{c^{2}} \frac{x_{c}}{y_{c}} .
$$
then $R$ is found using $x_{c}\left(y_{c}\right)$. For masses larger than $M_{\max }$, the radius limit is $R>2.823 G M / c^{2}$. This result is indicated in Fig. 2 assuming $M_{\max }=1.97 M_{\odot}$ and in Fig. 3 for $M_{\max }=2.01 M_{\odot}$ and $2.4 M_{\odot}$. Fig. 2 shows that a number of EOSs are ruled out by the observation that $M_{\max } \gtrsim 2 M_{\odot}$ stars. The green 285 neutron stars be found, progressivly larger $M-R$ regions become inaccessible. 


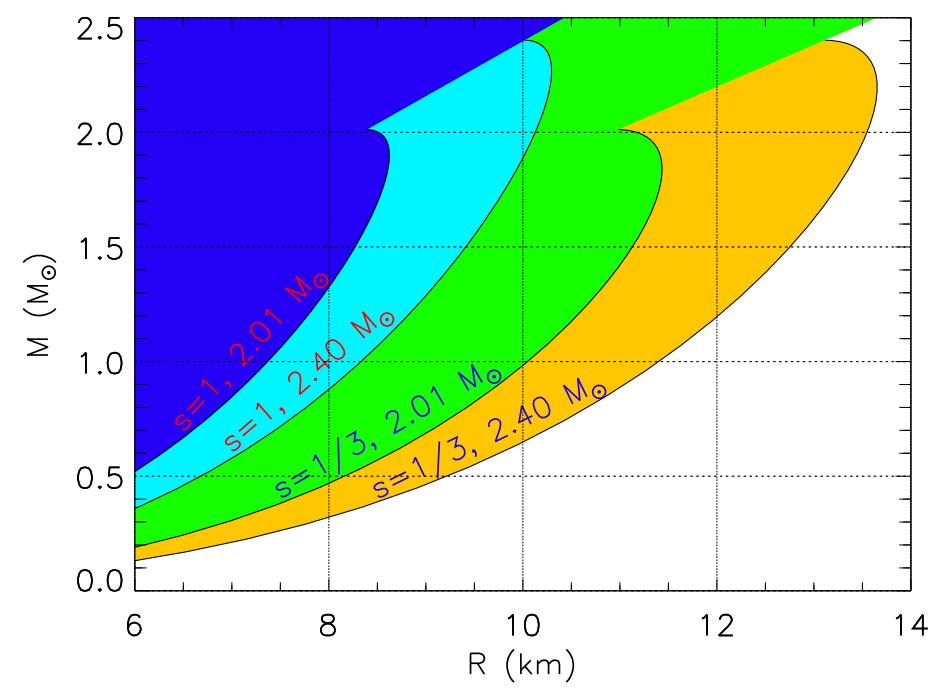

Figure 3: Mass-radius diagram showing regions excluded by causality and the maximally compact conjecture. Boundaries are indicated for two values of $M_{\max }\left(2.01 M_{\odot}, 2.4 M_{\odot}\right)$ and for two values of sound speed squared $s(1,1 / 3)$.

Fig. 4 shows the limiting radii for a typical $1.4 M_{\odot}$ star as a function of $M_{\max }$. Also displayed is the equivalent boundary if the constraint $s=1 / 3$ is alternately employed. A phenomenolgical result noted by Ref. [45] is that models of neutron stars containing deconfined quark matter or cores with a mixed quark-hadron phase instead have radii limited by the maximally compact EOS assuming $s=$ $1 / 3$, i.e., $p=\left(\epsilon-\epsilon_{0}\right) / 3$ for $\epsilon>\epsilon_{0}$. This constraint changes the eigenvalues $\left[w_{0, c}=4.826, x_{1, c}=0.1910\right.$ and $\left.y_{1, c}=0.05169\right]$ and dramatically increases the limiting radii, as shown in Fig. 3. This limit, as shown below, might be closer to the the radii of realistic EOSs based on experimental studies than is the $s=1$ boundary, but there are no physical grounds for taking it as a limit. It is useful to display the limiting radii for $1.4 M_{\odot}$ stars as a function of $M_{\max }$, and this is done in Fig. 4.

The limiting radii provided by the $s=1$ assumption, while interesting, assumed $p=0$ at low densities and can be improved with constraints on the low-density EOS. Neutron stars are believed to have crusts, and experimental and theoretical information can be brought to bear on the properties of matter near $n_{s}$. In the following, we make use of these constraints to examine refined, 


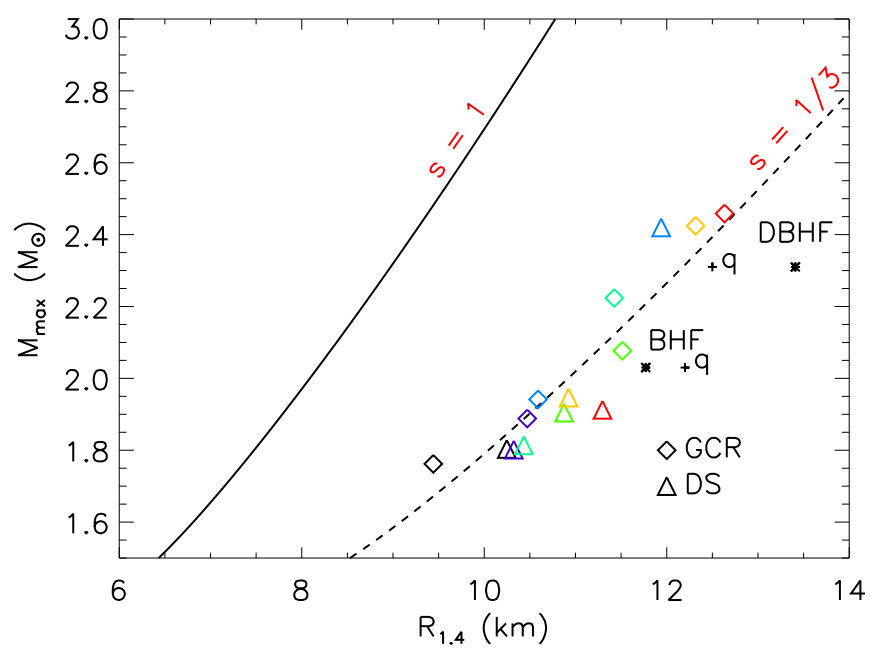

Figure 4: The minimum radii of $1.4 M_{\odot}$ stars, as a function of $M_{\max }$, permitted by causality and the maximum compactness conjecture. Results are shown for $s=1 / 3$ and $s=1$. The symbols show the maximum massses and values of $R_{1 / 4}$ obtained from TOV integrations after extrapolating different neutron matter calculations from Refs. 46, 47] using Eq. 22, to arbitrarily high densities.

model-independent limits to neutron star radii.

\subsection{The Neutron Star Crust and the Low-Density EOS}

There are many observations that indicate most, if not all, neutron stars have hadronic crusts, i.e., they have a surface region with densities less than approximately $10^{14} \mathrm{~g} \mathrm{~cm}^{-3}$ composed largely of nuclei, neutrons and electrons in beta equilibrium [48, 49]. Observations of pulsar glitches [50, neutron star cooling following transient heating events [51, and the general consistency of observed thermal emissions from cooling neutron stars [52 favor the existence of a hadronic crust 7 The pressure in the crust is largely due to relativistic, degenerate electrons with at most a $5 \%$ contribution from nuclei and neutrons.

\footnotetext{
${ }^{7}$ There exist, however, alternative interpretations of these observations involving a thin $\left(M \simeq 10^{-5} M_{\odot}\right)$, electrostatically-supported, hadronic shell overlaying a high-density pure strange quark star (also known as a self-bound star) [53 [54].
} 
Since the nuclei are in pressure equilibrium with the neutrons, they individually

contribute almost no pressure since their internal baryon density is close to the nuclear saturation density $n_{s} \simeq 0.16 \mathrm{fm}^{-3}$ or $\rho_{s} \simeq 2.7 \times 10^{14} \mathrm{~g} \mathrm{~cm}^{-3}$ where uniformly dense symmetric matter has zero pressure. The major contribution of baryons to the pressure is from the collective Coulomb pressure due to the nuclear lattice, and is therefore largely independent of uncertainties in the nuclear matter EOS.

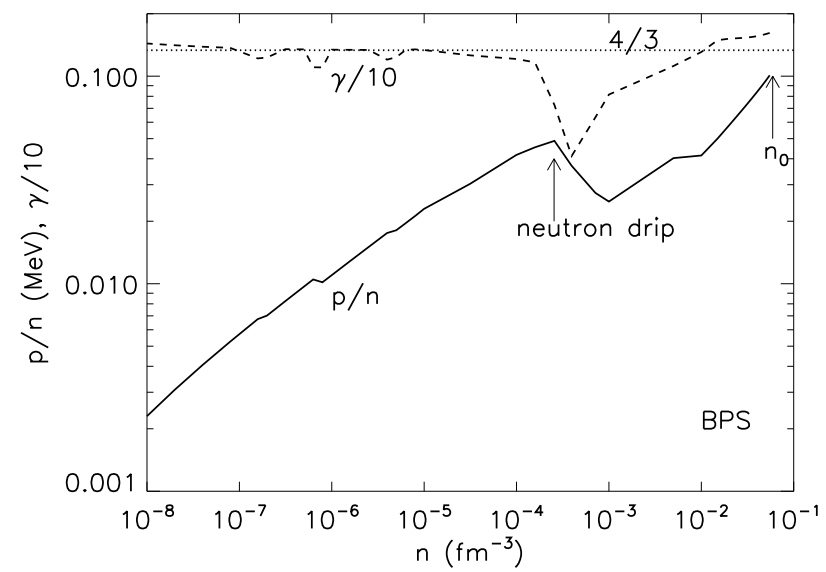

Figure 5: The BPS 48] EOS. The solid line is the pressure, and the dashed line is the polytropic exponent. The value corresponding to $\gamma=4 / 3$ is shown as the dotted line. The neutron drip density and the core-crust transition density $n_{0}$ are shown.

Although the average value of $\gamma$ in the crust, as expected, is about $4 / 3$, it drops significantly following the onset of neutron drip at a density $4.3 \cdot 10^{11} \mathrm{~g}$ $\mathrm{cm}^{-3}$, due to the introduction of a new degree of freedom. It then recovers, and even exceeds $4 / 3$ at densities just below $n_{s}$. Fig. 5 shows the pressure and polytropic index as a function of density within the crust for the BPS EOS [48]. Estimates from neutron matter calculations [55] indicate that the transition from crustal material to uniform nuclear matter occurs in the range $0.4 \lesssim n_{0} / n_{s} \lesssim 0.5$. We will assume the crust-core transition density is $n_{0}=$ $n_{s} / 2.7$, and use the BPS [48] crustal EOS, for which the pressure, energy density 
and internal energy per baryon at $n_{0}$ are

$$
p_{0}=0.243 \mathrm{MeV} \mathrm{fm}^{-3}, \quad \epsilon_{0}=56.39 \mathrm{MeV} \mathrm{fm}^{-3}, \quad E_{0}=12.13 \mathrm{MeV}
$$

Results are relatively insensitive to the exact location of the core-crust boundary.

\subsection{The Intermediate EOS and Pure Neutron Matter}

Matter in neutron stars at densities between $n_{0}$ and at least $2 n_{s}$ is most likely a structureless fluid of nucleons which is extremely neutron rich due to the condition of beta equilibrium. This condition is equivalent to minimization of the total energy per baryon with respect to the charge fraction $x=n_{p} / n$ where $n_{n}$ and $n_{p}$ are the neutron and proton baryon densities, respectively, and $n=n_{n}+n_{p}$. The difference between the energy of pure neutron matter and symmetric matter (with equal numbers of neutrons and protons) is called the nuclear symmetry energy $S(n)$, and the energies of intermediate proton fractions can be approximated with a quadratic interpolation between these extremes:

$$
E(n, x) \simeq E_{1 / 2}(n)+S(n)(1-2 x)^{2},
$$

where $E_{1 / 2}(n)$ is the energy per baryon of symmetric matter. We define $E_{1 / 2}\left(n_{s}\right)=$ $-B$, where $B \simeq 16 \mathrm{MeV}$ is the bulk binding energy per baryon of symmetric matter at the saturation density. We make the distinction here between $S(n)$ and $S_{2}(n)$ defined by

$$
S_{2}(n)=(1 / 8)\left(\partial^{2} E(n, x) / \partial x^{2}\right)_{x=1 / 2}
$$

In many treatments, $S_{2}$ is also called the symmetry energy, but only if the quadratic approximation is valid will $S(n)=S_{2}(n)$. A convenient estimate for the symmetry energy near $n_{s}$ is

$$
S(n)=S_{v}\left(n / n_{s}\right)^{\gamma}
$$

$S_{v}=S_{2}\left(n_{s}\right)$. Nuclear experimental information and neutron matter calculations indicate that $26 \mathrm{MeV} \lesssim S_{v} \lesssim 34 \mathrm{MeV}$ and $0.3 \lesssim \gamma \lesssim 0.7$, with the value of $\gamma$ 
positively correlated with $S_{v}$. The pressure corresponding to Eqs. 14 and (16) is

$$
p(n, x)=n^{2} \frac{\partial E(n, x)}{\partial n} \simeq p_{1 / 2}(n)+S_{v} \gamma n_{s}\left(\frac{n}{n_{s}}\right)^{\gamma+1}(1-2 x)^{2},
$$

where $p_{1 / 2}(n)$ is the pressure of symmetric matter. Note that, by definition, $p_{1 / 2}\left(n_{s}\right)=0$; to leading order, near $n_{s}$, the symmetric matter pressure increases linearly with density, $p_{1 / 2}(n) \simeq\left(K_{s} / 9\right)\left(n-n_{s}\right)$.

Matter in neutron stars is in beta equilibrium with $\mu_{n}-\mu_{p}=\mu_{e}$, which follows from minimizing the total baryon and electron energies with respect to the proton fraction $x$ :

$$
\partial\left[E(n, x)+E_{e}(n, x)\right] / \partial x=0 .
$$

$E_{e}=(3 / 4) \hbar c x\left(3 \pi^{2} n x\right)^{1 / 3}$ is the electron energy per baryon assuming relativistic degeneracy. For the symmetry energy ansatz Eq. (16), applicable to uniform nucleonic matter, this is equivalent to

$$
4 S_{v}\left(n / n_{s}\right)^{\gamma}(1-2 x)=\hbar c\left(3 \pi^{2} n x\right)^{1 / 3} .
$$

This can be solved as a cubic equation for $x$ at a specific density, but since $x$ is small, has the approximate solution

$$
x_{\beta} \simeq \frac{64 S_{v}^{3}\left(n / n_{s}\right)^{3 \gamma}}{3 \pi^{2} n_{s}(\hbar c)^{3}+384 S_{v}^{3}\left(n / n_{s}\right)^{3 \gamma}}
$$

which has the value $x_{\beta} \simeq 0.040$ when $n=n_{s}$ and $\left.S_{v}=31 \mathrm{MeV}\right]^{8}$ The pressure of pure neutron matter using Eq. (16), at $n_{s}$, is $p\left(n_{s}\right)=\gamma n_{s} S_{v}$. In beta equilibrium, to lowest orders, this is modified:

$$
p_{\beta}\left(n_{s}\right) \simeq \gamma n_{s} S_{v}\left[1-\left(\frac{4 S_{v}}{\hbar c}\right)^{3} \frac{4 \gamma-1}{3 \pi^{2} n_{s} \gamma}\right] .
$$

The correction term in Eq. 21] is of order 1.4\%, and can be ignored to good approximation. At higher densities, the proton fraction and the correction term

\footnotetext{
${ }^{8}$ In reality, muons should be included because $\mu_{e}=\hbar c\left(3 \pi^{2} n_{s} x_{\beta}\right)^{1 / 3} \simeq 113 \mathrm{MeV}>m_{\mu} c^{2} \simeq$ $105 \mathrm{MeV}$. This omission has little effect on our discussion.
} 
generally increase due to the increasing symmetry energy. There is also a contribution from $p_{1 / 2}(n)$. However, for densities up to $2 n_{s}$ the neutron star matter pressure is essentially equivalent to pure neutron matter pressure.

\begin{tabular}{|c||c|c|c|c||c|c|c|c|}
\hline Model & $\begin{array}{c}a \\
\mathrm{MeV}\end{array}$ & $\alpha$ & $\begin{array}{c}b \\
\mathrm{MeV}\end{array}$ & $\beta$ & $\begin{array}{c}S_{v} \\
\mathrm{MeV}\end{array}$ & $\begin{array}{c}L \\
\mathrm{MeV}\end{array}$ & $\begin{array}{c}p_{1} \\
\mathrm{MeV} \mathrm{fm}^{-3}\end{array}$ & $\gamma_{1}$ \\
\hline GCR 0 & 12.7 & 0.49 & 1.78 & 2.26 & 30.5 & 31.3 & 7.272 & 2.113 \\
GCR 1 & 12.7 & 0.48 & 3.45 & 2.12 & 32.1 & 30.8 & 10.402 & 2.335 \\
GCR 2 & 12.8 & 0.488 & 3.19 & 2.20 & 32.0 & 40.6 & 10.537 & 2.343 \\
GCR 3 & 13.0 & 0.475 & 3.21 & 2.47 & 32.0 & 44.0 & 13.274 & 2.487 \\
GCR 4 & 12.6 & 0.475 & 5.16 & 2.12 & 33.7 & 51.5 & 14.304 & 2.533 \\
GCR 5 & 13.0 & 0.50 & 4.71 & 2.49 & 33.8 & 56.2 & 18.678 & 2.700 \\
GCR 6 & 13.4 & 0.514 & 5.62 & 2.436 & 35.1 & 63.6 & 20.933 & 2.770 \\
\hline DHS 0 & 10.94 & 0.459 & 4.106 & 1.977 & 31.1 & 39.4 & 8.125 & 2.182 \\
DHS 1 & 11.00 & 0.460 & 4.425 & 1.947 & 31.4 & 41.0 & 8.453 & 2.206 \\
DHS 2 & 11.95 & 0.495 & 3.493 & 2.632 & 31.4 & 45.3 & 13.760 & 2.509 \\
DHS 3 & 11.02 & 0.460 & 4.683 & 1.935 & 31.7 & 42.4 & 8.768 & 2.229 \\
DHS 4 & 10.95 & 0.454 & 5.158 & 1.972 & 32.1 & 45.4 & 9.676 & 2.290 \\
DHS 5 & 10.34 & 0.429 & 4.954 & 2.024 & 31.3 & 43.4 & 9.180 & 2.258 \\
DHS 6 & 10.29 & 0.433 & 7.227 & 1.842 & 33.5 & 53.3 & 11.241 & 2.384 \\
\hline
\end{tabular}

Table 2: Neutron matter calculations fit to the energy parameterization of Eq. 22. GCR are models from Ref. 46]; DHS are models from Drischler and Schwenk (2015, unpublished).

Recent calculations of the properties of pure neutron matter have produced estimates of the pressure-energy density relation up to about $2 n_{s}$. Ref. [46] showed that the neutron matter energy for densities less than about $2 n_{s}$ was adequately approximated by the double power law

$$
E(n, 0) \simeq a\left(n / n_{s}\right)^{\alpha}+b\left(n / n_{s}\right)^{\beta}
$$

where $a, b, \alpha$ and $\beta$ are parameters. Table 2 displays parameter values found by Ref. 46 for quantum Monte Carlo neutron matter calculations. We have also 
displayed parameter values that fit the neutron matter results of unpublished calculations of Drischler \& Schwenk up to densities $\simeq 1.5 n_{s}$.

Naively, we can extend neutron matter calculations to arbitrarily higher densities using Eq. 222. Doing so, and using the TOV equations to produce $M-R$ curves, we find many of the neutron matter calculations in Table 2 to be too soft to support the observed value of $M_{\max }$ (Fig. 44). It appears that the

EOS must become substantially stiffer at densities not far above $n_{s}$ in order that observed neutron star masses can be explained. Nevertheless, it is interesting to observe that $M_{\max }$ and $R_{1.4}$ for these extrapolations are near the $s=1 / 3$ boundary for the maximally compact EOS.

\section{Nuclear Structure and the Nuclear Symmetry Energy}

To confirm the understanding of the intermediate EOS, we turn to experimental data for nuclei which explore the nuclear symmetry energy. Experimental information concerning the symmetry energy is usually encoded in the parameters $S_{v}$ and $L$, defined as

$$
\begin{aligned}
S_{v} & \equiv \frac{1}{8}\left(\frac{\partial^{2} E(n, x)}{\partial x^{2}}\right)_{n_{s}, 1 / 2} \simeq S\left(n_{s}\right) \\
L & \equiv \frac{3}{8}\left(\frac{\partial^{3} E(n, x)}{\partial n \partial x^{2}}\right)_{n_{s}, 1 / 2} .
\end{aligned}
$$

For the symmetry energy of Eq. (16), one finds $L=3 \gamma S_{v}$ so that $0.9 S_{v} \lesssim$ $L \lesssim 2.1 S_{v}$. For the energy formula Eq. 222 , we find that $S_{v}=B+a+b$ and $L=3(a \alpha+b \beta)$. For each set of neutron matter calculations, the corresponding values of $S_{v}, L$ have been tabulated in Table 2, which reveals that $30.5 \mathrm{MeV}$ $\leq S_{v} \leq 35.1 \mathrm{MeV}$ and $30.8 \mathrm{MeV} \leq L \leq 63.6 \mathrm{MeV}$. As anticipated, these

350 parameters are positively correlated. In the quadratic approximation for the isospin dependence of the nucleon energies, $L=3 p\left(n_{s}, 0\right) / n_{s}$.

\subsection{Hydrodynamic Model of the Nuclear Symmetry Energy}

A variety of experiments reveal information about the nuclear symmetry energy. None of these experiments are capable, at the present time, of individ- 
Lipparini \& Stringari [56] explored a model with a simplified nuclear Hamiltonian energy density

$$
\begin{aligned}
\mathcal{H} & =\mathcal{H}_{B}(n, \alpha)+\mathcal{Q}(n)(d n / d r)^{2} \\
\mathcal{H}_{B}(n, \alpha) & =\mathcal{H}_{B}(n, 0)+v_{\text {sym }}(n) \alpha^{2}
\end{aligned}
$$

where the uniform matter contribution is $\mathcal{H}_{B}(n, \alpha), \mathcal{Q}(n)$ controls the gradient contributions, $v_{\text {sym }}=S_{2} / n$, and $\alpha=n-2 n_{p}=n_{n}-n_{p}$ is the isovector density. In nuclei, the charge repulsion among the protons redistributes neutrons and protons and reduces the neutron skin thickness. To take this into account, and to extend the model of Ref. [56, we include a Coulomb contribution:

$$
\mathcal{H}=\mathcal{H}_{B}(n, \alpha)+\mathcal{H}_{C}(n, \alpha)+\mathcal{Q}(n)(d n / d r)^{2},
$$

where, in spherical symmetry, $\mathcal{H}_{C}=n_{p} V_{C} / 2$ and the Coulomb potential is

$$
V_{C}(r)=\frac{e^{2}}{r} \int_{0}^{r} n_{p}\left(r^{\prime}\right) d^{3} r^{\prime}+\int_{r}^{\infty} \frac{e^{2}}{r^{\prime}} n_{p}\left(r^{\prime}\right) d^{3} r^{\prime} .
$$

If the protons are uniformly distributed for $r<R$,

$$
V_{C}=\frac{Z e^{2}}{R}\left(\frac{3}{2}-\frac{r^{2}}{2 R^{2}}\right)
$$

for $r<R$ and $V_{C}=Z e^{2} / r$ for $r>R$. We have found that a reasonable approximation for a Woods-Saxon proton distribution, and one that keeps the model analytic, is provided by assuming Eq. (27) to apply for all $r$. Furthermore, the Coulomb potential and the total Coulomb energy when the Coulomb potential is self-consistently determined are adequately described by the same approximation. Where the discrepancy between this approximation and the real potential is large, the proton density is small. 
We now optimize the total nuclear energy with respect to the densities $n$ and $\alpha$ subject to the constraints

$$
A=\int \rho d^{3} r, \quad N-Z=\int \alpha d^{3} r
$$

producing the chemical potentials $\mu$ and $\bar{\mu}$ :

$$
\frac{\delta}{\delta n}[\mathcal{H}-\mu n]=0, \quad \frac{\delta}{\delta \alpha}[\mathcal{H}-\bar{\mu} \alpha]=0 .
$$

These lead to

$$
\begin{aligned}
\mu & =\frac{\partial\left[\mathcal{H}_{B}+\mathcal{H}_{C}\right]}{\partial n}-2 \frac{d}{d r}\left[\mathcal{Q} n^{\prime}\right]+\frac{\partial \mathcal{Q}}{\partial n}\left(n^{\prime}\right)^{2} \\
\bar{\mu} & =\frac{\partial\left[\mathcal{H}_{B}+\mathcal{H}_{C}\right]}{\partial \alpha}
\end{aligned}
$$

\subsubsection{The isoscalar density $n$}

Multiplying the first of Eq. 29 by $d n / d r$ and the second by $d \alpha / d r$, their sum can be integrated:

$$
\mathcal{Q}(n)(d n / d r)^{2}=\mathcal{H}_{B}(n, \alpha)+\mathcal{H}_{C}(n, \alpha)-\mu n-\bar{\mu} \alpha,
$$

for which the boundary condition $\mu n_{o}+\bar{\mu} \alpha_{o}=\mathcal{H}_{B}\left(n_{o}, \alpha_{o}\right)$ at the center.

We make the quadratic approximation for the energy density of uniform symmetric matter:

$$
\mathcal{H}_{B}(n, 0)=n\left[-B+\frac{K_{s}}{18}(1-u)^{2}\right]
$$

with bulk binding energy $B$, and $u=n / n_{s}$. In the case that $\alpha_{o} \simeq 0$, one has $n_{o}=n_{s}=0.16 \mathrm{MeV} \mathrm{fm}^{-3}$. For laboratory nuclei, $\alpha_{o}$ is small. We are primarily interested in the behavior of $\alpha$, and will assume that the total density, to lowest order, can be found by assuming $\alpha \simeq 0$ in Eq. (31). We also approximate $\mathcal{Q}(n)=Q / n$, with $Q$ constant, which leads to an equation for the isoscalar density as a function of position:

$$
\frac{d u}{d z}=-u(1-u), \quad a=3 \sqrt{\frac{2 Q}{K_{s}}},
$$


where $u=n / n_{o}$ and $z=r / a$, where the surface thickness parameter is $a$. This has the solution of a Fermi function, or Woods-Saxon distribution (which was assumed by Ref. [56]),

$$
u=\frac{1}{1+e^{z-y}} .
$$

Here $y$ is a constant of integration, determined from the first of the constraints

375

$$
\begin{aligned}
A & =\int n d^{3} r=4 \pi n_{o} a^{3} F_{2}(y), \\
F_{i}(y) & =\int_{0}^{\infty} \frac{z^{i} d z}{1+e^{z-y}} \simeq \frac{y^{i+1}}{i+1}\left[1+\frac{i(i+1)}{6}\left(\frac{\pi}{y}\right)^{2}+\cdots\right] .
\end{aligned}
$$

Here $F_{i}$ is the usual Fermi integral, and the right-most approximation holds for $y>>1$ and $i \neq-1$ if we ignore an exponentially small term. This is justified, since one finds [ Eq. [35] that $y \simeq R / a \simeq 13$ for ${ }^{208} \mathrm{~Pb}$ ( $a$ is evaluated below).

The parameter $K_{s} \simeq 240 \mathrm{MeV}$ from experiment [5, 6, 7, and the value of $Q$ follows from the observed value[57] of the 90-10 surface thickness:

$$
t_{90-10}=a \int_{0.1}^{0.9} \frac{d u}{d u / d z}=4 a \ln (3) \simeq 2.3 \mathrm{fm},
$$

giving $a=0.523 \mathrm{fm}$ and

$$
Q=\frac{K_{s}}{18}\left(\frac{t_{90-10}}{4 \ln (3)}\right)^{2} \simeq 3.65 \mathrm{MeV} \mathrm{fm}^{2} .
$$

As a check, the liquid droplet surface tension parameter is the semi-infinite, symmetric matter, surface thermodynamic potential per unit area:

$$
\begin{aligned}
\sigma_{o} & =\int[\mathcal{H}-\mu n] d z=2 Q \int_{0}^{\infty} \frac{1}{n}\left(\frac{d n}{d z}\right)^{2} d z \\
& =\frac{2 Q n_{o}}{a} \int_{0}^{1}(1-u) d u=\frac{Q n_{o}}{a} \simeq 1.17 \mathrm{MeV} \mathrm{fm}^{-2}
\end{aligned}
$$

This gives a value $E_{s}=4 \pi r_{o}^{2} \sigma_{o} \simeq 19.2 \mathrm{MeV}$, with $n_{o}=4 \pi r_{o}^{3} / e$, for the surface energy parameter in the liquid droplet model, very close to the accepted value [58, 57. Therefore, the simple approximations Eq. (32) and $\mathcal{Q}=Q / n$ fit the most important observed properties of the symmetric matter surface, its tension and thickness, as well as the matter's observed incompressibility. 


\subsubsection{The isovector density $\alpha$}

Using the second of the variations Eq. (30) leads to

$$
\alpha=\frac{\bar{\mu}+\partial \mathcal{H}_{C} / \partial \alpha}{2 v_{\text {sym }}}=\frac{\bar{\mu}+V_{C} / 2}{v_{\text {sym }}},
$$

where we note that $n_{p} V_{C}$ is proportional to $n_{p}^{2}=(n-\alpha)^{2} / 4$ and we treated $n_{p} V_{C}$ to be primarily a function of $\alpha$ and not $n$. This can be justified on the grounds that while the Coulomb potential acts directly on the protons only, the neutrons adjust due to the strong symmetry interaction.

Using the second of the constraints (Eq. (28)), we obtain

$$
N-Z=\int \frac{\bar{\mu}+V_{C} / 2}{2 v_{\text {sym }}} d^{3} r \equiv \frac{\bar{\mu}}{2} H_{0}+\frac{G}{4},
$$

where we define $H_{i}$ and $G$ as

$$
H_{i}=\int\left(\frac{r}{R}\right)^{i} \frac{d^{3} r}{v_{\text {sym }}}, \quad G=\int \frac{V_{c}}{v_{\text {sym }}} d^{3} r=\frac{Z e^{2}}{2 R}\left(3 H_{0}-H_{2}\right) .
$$

It then follows that

$$
\alpha=\frac{N-Z-\left(G-V_{C} H_{0}\right) / 4}{v_{\text {sym }} H_{0}} .
$$

\subsubsection{Nuclear structure}

The total symmetry and Coulomb energy is

$$
\begin{aligned}
E_{\text {sym }}+E_{C} & =\int v_{\text {sym }} \alpha^{2} d^{3} r+\frac{1}{4} \int(n-\alpha) V_{C} d^{3} r \\
& =\frac{(N-Z)^{2}}{H_{0}}-\frac{(N-Z) G}{4 H_{0}}+\frac{1}{4} \int n V_{C} d^{3} r \\
& =\frac{(N-Z)^{2}}{H_{0}}+\frac{3}{5} \frac{Z^{2} e^{2}}{R}+\frac{Z e^{2}}{8 R}(N-Z)\left(\frac{H_{2}}{H_{0}}-\frac{3}{5}\right) .
\end{aligned}
$$

We used $\int n r^{2} d^{3} r \simeq 3 A R^{2} / 5$ which follows from Eq. (35) to lowest order. ${ }^{9}$

The dipole static polarizability, $\alpha_{D}$, is found by performing the constrained variation 56 ]

$$
\frac{\delta}{\delta \alpha}\left(\int \mathcal{H} d^{3} r-\epsilon \int z \alpha d^{3} r\right)=0
$$

\footnotetext{
${ }^{9}$ The total symmetry and Coulomb energy is independent of including polarization effects on $\alpha$, i.e., treating $\mathcal{H}_{\mathcal{C}}$ to be a function of $\alpha$.
} 
with $\epsilon$ a small parameter. Defining $\alpha_{d}$ as the function $\alpha(r)$ which solves Eq. 44 . the dipole polarizability is

$$
\alpha_{D}=\frac{1}{2 \epsilon} \int z \alpha_{d} d^{3} r
$$

The solutions for $\alpha_{d}$ and the dipole polarizability are

$$
\alpha_{d}=\frac{\epsilon z+V_{C}}{2 v_{\text {sym }}}, \quad \alpha_{D}=\frac{1}{4 \epsilon} \int z \frac{\epsilon z+V_{C}}{v_{\text {sym }}} d^{3} r=\frac{R^{2} H_{2}}{12},
$$

where $z^{2}=r^{2} / 3$ within the integral. ${ }^{10}$

From Eqs. 42 and (41), the central isovector density is

$$
\alpha_{o}=\frac{N-Z-G / 4+V_{C, o} H_{0} / 4}{v_{\text {sym }, o} H_{0}}=\frac{n_{o}}{S_{v} H_{0}}\left[N-Z+\frac{Z e^{2}}{8 R} H_{2}\right],
$$

where $v_{\text {sym }, o}=S_{v} / n_{o}$ and $V_{C, o}$ are central values. Polarization thus results in an increase in asymmetry near the nuclear center.

The neutron skin thickness $R_{n}-R_{p}$, the difference between the mean radii of neutrons and protons, is defined by

$$
\frac{4 \pi}{3}\left(R_{n}^{3}-R_{p}^{3}\right)=\int\left(\frac{n_{n}}{n_{n o}}-\frac{n_{p}}{n_{p o}}\right) d^{3} r
$$

where $n_{n o}$ and $n_{p o}$ are the central values of the neutron and proton densities. $R_{n}$ and $R_{p}$ represent the "squared-off" radii. We find

$$
\frac{n_{n}}{n_{n o}}-\frac{n_{p}}{n_{p o}}=\frac{n_{o}}{2 n_{n o} n_{p o}}\left(\alpha-n \frac{\alpha_{o}}{n_{o}}\right)
$$

and

$$
\frac{4 \pi}{3}\left(R_{n}^{3}-R_{p}^{3}\right)=\frac{n_{o} A}{2 n_{n o} n_{p o}}\left(I-\frac{\alpha_{o}}{n_{o}}\right)
$$

Treating $R_{n}-R_{p}<<R$ and keeping the lowest-order term:

$$
\frac{R_{n}-R_{p}}{R} \simeq \frac{2}{3} \frac{I-\alpha_{o} / n_{o}}{1-\alpha_{o}^{2} / n_{o}^{2}}=\frac{2}{3}\left[I\left(1-\frac{A}{S_{v} H_{0}}\right)-\frac{Z e^{2}}{8 R S_{v}} \frac{H_{2}}{H_{0}}\right],
$$

where $I=(N-Z) / A$. The denominator term can be neglected since $I$ and $\alpha_{o} / n_{o}$ are of the same small magnitude. Polarization effects obviously decrease the neutron skin thickness 11

\footnotetext{
${ }^{10}$ The second term in the middle expression for $\alpha_{D}$ in Eq. 46 vanishes because of symmetry, so that this result is also independent of including polarization effects on $\alpha$.

${ }^{11}$ In the case of a symmetric nucleus, there is, in fact, a proton skin.
} 
Experimentally, however, it is preferable to measure the differences of the mean-square neutron and proton radii, which, if the densities are uniform up to $R_{n}$ or $R_{p}$, is $\sqrt{3 / 5}\left(R_{n}-R_{p}\right)$. These radii, in the hydrodynamical model, are

$$
\begin{aligned}
r_{n, p}^{2} & =\frac{1}{(N, Z)} \int n_{n, p} r^{2} d^{3} r=\frac{1}{2(N, Z)} \int(n \pm \alpha) r^{2} d^{3} r \\
& =\frac{R^{2}}{1 \pm I}\left[\frac{3}{5} \pm\left(\frac{I H_{2}}{H_{0}}+\frac{Z e^{2}}{8 R A}\left(\frac{H_{2}^{2}}{H_{0}}-H_{4}\right)\right)\right],
\end{aligned}
$$

where the upper (lower) sign refers to $n(p)$. We then find

$$
\frac{r_{n p}}{R} \simeq \sqrt{\frac{3}{5}}\left[I\left(\frac{5 H_{2}}{3 H_{0}}-1\right)+\frac{5 Z e^{2}}{24 R A}\left(\frac{H_{2}^{2}}{H_{0}}-H_{4}\right)\right]\left(1-I^{2}\right)^{-1 / 2}
$$

\subsubsection{Solutions for arbitrary $v_{\text {sym }}$}

Lattimer \& Steiner [59] noted that if $v_{s y m}$ is expanded according to

$$
\frac{S_{v}}{n_{o} v_{s y m}(u)}=\sum_{j=1}^{J} b_{j} u^{j}, \quad 0<u<1
$$

and the density has the Fermi shape of Eq. (34), the $H_{i}$ integrals are analytic:

$$
H_{i}=\int\left(\frac{r}{R}\right)^{i} \frac{d^{3} r}{v_{s y m}}=\frac{4 \pi n_{o} a^{3+i}}{S_{v} R^{i}}\left[F_{i+2}(y)-(2+i) \mathcal{T} F_{i+1}(y)+\cdots\right],
$$

where

$$
\mathcal{T}=b_{2}+3 b_{3} / 2+11 b_{4} / 6+25 b_{5} / 12+137 b_{6} / 60+\cdots
$$

Note that $\sum_{j=1}^{J} b_{j}=1$ ensures $v_{\text {sym }}(u=1)=S_{v} / n_{o}$. In principle, any $v_{\text {sym }}$ can be fit if $J$ is large enough, but as a simple example, consider a three term fit to the conventional expression of the symmetry energy

$$
S_{2}(u) \simeq S_{v}+\frac{L}{3}(u-1)+\frac{K_{\text {sym }}}{18}(u-1)^{2}+\cdots ;
$$


if $K_{\text {sym }}=18\left(L / 3-S_{v}\right) \simeq-210 \mathrm{MeV}$, then $S_{2}(0)$ approximately vanishes. Fitting the energy and its first two derivatives at $u=1$ give ${ }^{12}$

$$
\begin{aligned}
b_{1} & =1+\frac{L}{3 S_{v}}+\left(\frac{L}{3 S_{v}}\right)^{2}-\frac{K_{\text {sym }}}{18 S_{v}} \simeq 2.37, \\
b_{2} & =\frac{K_{\text {sym }}}{9 S_{v}}-\frac{L}{3 S_{v}}-2\left(\frac{L}{3 S_{v}}\right)^{2} \simeq-2.14, \\
b_{3} & =\left(\frac{L}{3 S_{v}}\right)^{2}-\frac{K_{\text {sym }}}{18 S_{v}} \simeq 0.76, \\
\mathcal{T} & =-\frac{L}{3 S_{v}}-\frac{1}{2}\left(\frac{L}{3 S_{v}}\right)^{2}+\frac{K_{\text {sym }}}{36 S_{v}} \\
& =-\frac{1}{2}\left[1+\frac{L}{3 S_{v}}+\left(\frac{L}{3 S_{v}}\right)^{2}\right] \simeq-0.992 .
\end{aligned}
$$

In the last expression for $\mathcal{T}$ we used $K_{\text {sym }}=18\left(L / 3-S_{v}\right)$ following from assuming $S_{2}(0)=013$

It is now possible to make a connection with the liquid droplet model [58, [57]. Without polarization corrections, the total symmetry energy of a nucleus in the liquid droplet model is

$$
E_{\text {sym }}=\frac{S_{v} A I^{2}}{1+S_{s} A^{-1 / 3} / S_{v}},
$$

where $S_{s}$ is the surface symmetry parameter. In the hydrodynamic model, this energy is $A^{2} I^{2} / H_{0}$ according to Eq. 43. Using Eq. (55) to leading orders,

$$
H_{0}=\frac{A}{S_{v}}\left(1+\frac{S_{s}}{S_{v} A^{1 / 3}}\right)=\frac{A}{S_{v}}\left(1-\frac{3 \mathcal{T} a}{r_{o} A^{1 / 3}}\right),
$$

showing that $S_{s}=-3 \mathcal{T} S_{v} a / r_{o}$. Therefore, once $v_{\text {sym }}$ is specified, the liquid droplet parameter $S_{s}$ can be calculated and the total symmetry and Coulomb energy, the dipole polarizability, and the neutron skin thickness can be found. For the above example, $S_{s} / S_{v} \simeq 1.36$. It also must follow that

$$
H_{i}=\frac{A}{S_{v}}\left(\frac{3}{3+i}+\frac{S_{s}}{S_{v} A^{1 / 3}}\right) .
$$

\footnotetext{
${ }^{12}$ The following solutions differ from those in Ref. [59].

${ }^{13}$ This fit to Eq. 57 is not very good for small $u$ since $\left[n v_{\text {sym }}(n)\right]_{n \rightarrow 0}=S_{v} / b_{1}$ which should, in fact, vanish; a more realistic fit with larger $J$ gives more negative values of $\mathcal{T}$.
} 
We can now form final expressions for the dipole polarizability and the neutron skin thickness:

$$
\begin{aligned}
\alpha_{D} & \simeq \frac{A R^{2}}{20 S_{v}}\left(1+\frac{5}{3} \frac{S_{s}}{S_{v} A^{1 / 3}}\right) \\
r_{n p} & \simeq \sqrt{\frac{3}{5\left(1-I^{2}\right)} \frac{2 r_{o}}{3}\left(1+\frac{S_{s}}{S_{v} A^{1 / 3}}\right)^{-1}\left[I \frac{S_{s}}{S_{v}}-\frac{3 Z e^{2}}{140 r_{o} S_{v}}\left(1+\frac{10}{3} \frac{S_{s}}{S_{v} A^{1 / 3}}\right)\right] .}
\end{aligned}
$$

\subsubsection{Predicted experimental correlations}

Nuclear experimental data concerning a single aspect of nuclear structure cannot pinpoint the symmetry parameters $S_{v}$ and $L$ uniquely. We first consider the correlation implied by nuclear binding energies [60, 61]. In the liquid droplet model [62, 58, 57, the total nuclear energy is

$$
E(N, Z)=-B A+E_{s} A^{2 / 3}+E_{\text {sym }}+E_{C}+E_{\text {pair }}+E_{\text {shell }} .
$$

Ignoring $E_{\text {pair }}$ and $E_{\text {shell }}$, the pairing and shell corrections which are globally small, the free parameters of this expression are $B$, the bulk binding energy per nucleon, $E_{s}$, the surface energy parameter, $r_{o}$, and $S_{v}$ and $S_{s}$ if we take Eq. (59) to represent the symmetry energy $E_{\text {sym }}$. By examining energy differences between nuclei with the same $A$ but different $N$ and $Z$, one can isolate $E_{\text {sym }}$ for many nuclear pairs. In reality, the total Coulomb and symmetry energies contain a term proportional to $I$ as seen in Eq. (43), but this term is generally small and we ignore it for this illustration. One can thus optimize the parameters $S_{s}$ and $S_{v}$ independently of the other liquid droplet parameters.

However, even though the binding energies of thousands of nuclei are precisely known, the range of values of $A^{1 / 3}$ for nuclei more massive than about $A=20$, large enough for the model to be applicable, is not large, varying from 3 to 6 . Therefore, only a correlation between $S_{s}$ and $S_{v}$ can be accurately determined. The correlation is very nearly linear between $S_{s} / S_{v}$ and $S_{v}$ and can be expressed as a confidence ellipse in the $S_{s} / S_{v}-S_{v}$ plane. One seeks to minize the quantity

$$
\chi^{2}=\sum_{i}^{N}\left(E_{\text {exp }, i}-E_{\text {sym }, i}\right)^{2} /\left(N \sigma^{2}\right),
$$


where $E_{e x p, i}$ and $E_{\text {sym }, i}$ (i.e., Eq. (59p) are the experimental and predicted energies of the $i$ th nuclear pair, respectively, $N$ is the total number of nuclear pairs, and $\sigma$ is a nominal error, usually taken to be about 1 to $2 \mathrm{MeV}$. A $\chi^{2}$ contour 1 unit above the minimum, which is the confidence ellipse, represents the $1 \sigma$ confidence level. The properties of this ellipse are determined by the second derivatives of $\chi^{2}$ at the minimum ${ }^{14}$

$$
\begin{aligned}
& \chi_{v v}=\frac{\partial^{2} \chi^{2}}{\partial S_{v}^{2}} \simeq \frac{2}{\mathcal{N} \sigma^{2}} \sum_{i}^{N} I_{i}^{4} A_{i}^{2}\left(1+\frac{S_{s}}{S_{v} A_{i}^{-1 / 3}}\right)^{-2}, \\
& \chi_{v s}=\frac{\partial^{2} \chi^{2}}{\partial S_{v} \partial\left(S_{s} / S_{v}\right)} \simeq \frac{2}{\mathcal{N} \sigma^{2}} \sum_{i}^{N} I_{i}^{4} A_{i}^{5 / 3} S_{v}\left(1+\frac{S_{s}}{S_{v} A_{i}^{-1 / 3}}\right)^{-3} \\
& \chi_{s s}=\frac{\partial^{2} \chi^{2}}{\partial\left(S_{s} / S_{v}\right)^{2}} \simeq \frac{2}{\mathcal{N} \sigma^{2}} \sum_{i}^{N} I_{i}^{4} A_{i}^{4 / 3} S_{v}^{2}\left(1+\frac{S_{s}}{S_{v} A_{i}^{-1 / 3}}\right)^{-4} .
\end{aligned}
$$

The orientation of the confidence ellipse with respect to the $S_{s} / S_{v}$ axis is $\theta=$ $(1 / 2) \tan ^{-1}\left[2 \chi_{v s} /\left(\chi_{v v}-\chi_{s s}\right)\right]$, and the error widths are $\sigma_{v}=\sqrt{\chi_{v v}^{-1}}$ and $\sigma_{s}=$ $\sqrt{\chi_{s s}^{-1}}$. The correlation coefficent is $\chi_{v s} / \sqrt{\chi_{v v} \chi_{s s}}$. Because the summations in Eq. (66) tend to be dominated by the more abundant heavier nuclei, we find

$$
\frac{\chi_{v v}}{\chi_{v s}} \simeq \sqrt{\frac{\chi_{v v}}{\chi_{s s}}} \simeq\left\langle A^{1 / 3}\right\rangle+\frac{S_{s}}{S_{v}}
$$

and so the slope of this correlation in $S_{v}-S_{s} / S_{v}$ space is about $7 \sqrt{15}$ It is apparent that the correlation between $S_{s}$ and $S_{v}$ is dependent on neither the model for the nucleus nor the nuclear force properties.

The inferred correlation between $L$ and $S_{v}$ is dependent upon assumptions about the nuclear interaction. For the model of Eqs. (57) and (58), $S_{s} / S_{v}$ depends only on $L / S_{v}$, so that

$$
\frac{d\left(S_{s} / S_{v}\right)}{d\left(L / S_{v}\right)}=\frac{d S_{s} / d S_{v}-S_{s} / S_{v}}{d L / d S_{v}-L / S_{v}} \simeq\left(a / 2 r_{o}\right)\left[1+2 L /\left(3 S_{v}\right)\right] \approx 0.5
$$

\footnotetext{
${ }^{14}$ We neglected terms containing $\left(E_{\text {exp }, i}-E_{\text {sym }, i}\right)$ in Eq. 66 as they are relatively small near the minimum.

${ }^{15} \mathrm{Had}$ we instead written the symmetry energy as $I_{i}^{2} A_{i}\left(S_{v}-S_{s} A^{-1 / 3}\right)$ as in the simple liquid drop model, the second derivatives would have been independent of the location of the minimum in $S_{s}-S_{v}$ space and the ellipse properties would have depended only upon the chosen values of $A_{i}$ and $I_{i}$. The slope of the correlation would then be $<A^{1 / 3}>\simeq 6$.
} 
and one obtains the slope $d L / d S_{v} \approx 12$ for the energy correlation in $S_{v}-L$ space.

In contrast to binding energies, which are available for over a thousand nuclei, measurements of giant resonances and skin thicknesses are more limited. Nevertheless, one can obtain correlations between $S_{s}$ and $S_{v}$ by considering individual measurements. In the case of the dipole polarizability, it is obvious from Eq. 62 that the resulting correlation in $S_{v}-S_{s} / S_{v}$ space will have a slope approximately $3 / 5$ that of the energy correlation. Without polarization corrections, the slope of the neutron skin thickness correlation would be zero, since Eq. (63) shows $r_{n p}$ would depend only on $S_{s} / S_{v}$. The slope of this correlation with polarization corrections included is actually negative, as can most easily be seen by considering the equations

$$
d\left(S_{s} / S_{v}\right)=a d L+b d S_{v}, \quad d r_{n p}=\alpha d\left(S_{s} / S_{v}\right)+\beta d S_{v} .
$$

Holding $r_{n p}$ fixed implies that the correlation slope in $S_{v}-S_{s} / S_{v}$ space would be negative if $\beta / \alpha>0$; the slope in $S_{v}-L$ space would be negative if $\beta / \alpha>-b$. From Eq. 63) we observe that, for ${ }^{208} \mathrm{~Pb}$,

$$
\frac{\beta}{\alpha}=\frac{3 Z e^{2}}{140 r_{o} S_{v}} \frac{\left(1+(10 / 3)\left(S_{s} / S_{v}\right) A^{-1 / 3}\right)\left(1+\left(S_{s} / S_{v}\right) A^{-1 / 3}\right)}{I-(7 / 3)\left(S_{s} / S_{v}\right) A^{-1 / 3}} \simeq 0.025
$$

so $S_{s} / S_{v}$ and $S_{v}$ are anticorrelated irrespective of the nuclear force model. On the other hand,

$$
b=-\frac{L}{S_{v}^{2}} \frac{d\left(S_{s} / S_{v}\right)}{d\left(L / S_{v}\right)}
$$

${ }^{420}$

which is sensitive to the interaction. For the simple interaction of Eq. (58), $b \simeq-0.03$ and in this case $r_{n p}$ has a positive correlation in $S_{v}-L$ space with a slope $d L / d S_{v} \simeq 0.3$ which is, nevertheless, nearly flat. Without polarization effects, we would have found $d L / d S_{v} \simeq 1.8$. Hartree Fock calculations [63, 39] with a variety of realistic interactions indicated that $d L / d S_{v}$ ranges from -3 to -5 . Irrespective of the exact value, it is clear that this correlation is nearly orthogonal to those from energies or dipole polarizabilities and is therefore extremely valuable in determining values for $S_{v}$ and $L$. 


\subsection{Symmetry Parameter Constraints from Experiment}

Measurements of all three types of observables, which have different correlations, can restrict the ranges of the symmetry parameters considerably. The experimental situation has been summarized in Refs. [45, 39] and is displayed in Fig. 6.

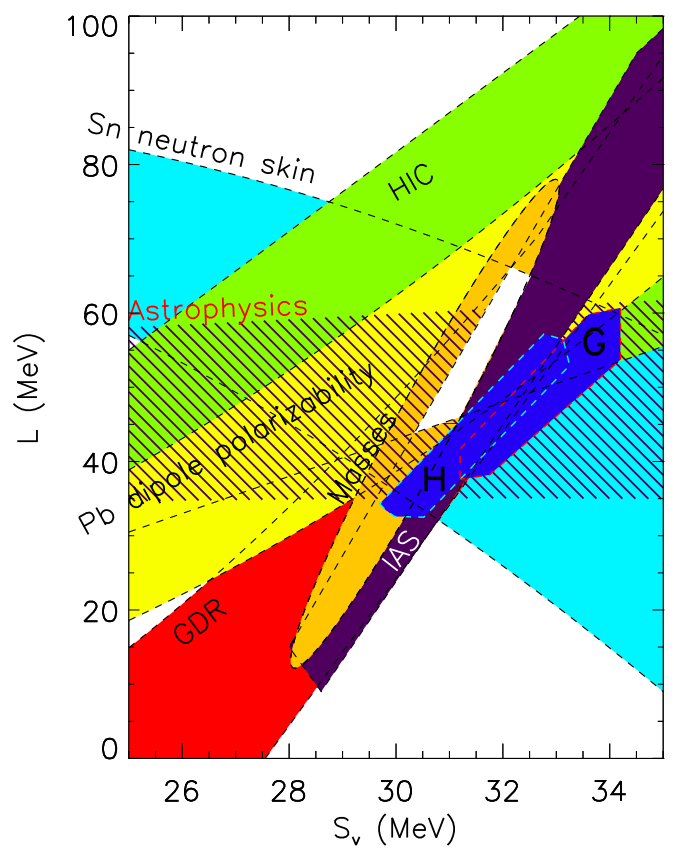

Figure 6: Experimental constraints for symmetry energy parameters, adapted and revised from Ref. 39]. See the text for further discussion and references to the experimental data and interpretation. $\mathrm{G}$ and $\mathrm{H}$ refer to the neutron matter studies of Gandolfi et al. [46] and Hebeler et al. 64, respectively.

The binding energy correlation (labelled "Masses") is taken from HartreeFock calulations with the UNEDF0 density functional [65, in which the nominal 435 fitting error was arbitrarily chosen to be $\sigma=1 \mathrm{MeV}{ }^{16}$ Importantly, and demonstrating the robustness of this result, the shape and orientation of the ellipse

\footnotetext{
${ }^{16}$ Ref. 65 had chosen $\sigma=2 \mathrm{MeV}$, but this value seems to be an overestimate, as negative values for $L$ would exist within the $1 \sigma$ confidence ellipse. Negative values of $L$ imply negative neutron matter pressures at $n_{s}$.
} 
are the same as predicted by the liquid droplet model, $d L / d S_{v} \approx 12$, once the dependence of $S_{s}$ on $S_{v}$ and $L$ is taken into account.

The constraints for the neutron skin thickness of ${ }^{208} \mathrm{~Pb}$ used in Fig. 6 are taken from a study by Chen et al. 63, who converted the experimental results [66, 67, 68, 69, 70, 71] for $\mathrm{Sn}$ isotopes into an equivalent value for ${ }^{208} \mathrm{~Pb}$ : $r_{n p} \simeq(0.175 \pm 0.020) \mathrm{fm}$. Assuming $S_{v}=31 \mathrm{MeV}$, the liquid droplet pediction, Eq. (63), is $S_{s} / S_{v}=3.0 \pm 0.4$. However, it is known that the liquid droplet model underestimates $r_{n p}$ because the neutron and proton distributions have different diffusenesses (i.e., different values of the surface thickness $a$ in Eq. (33) ) 72. For $\mathrm{Pb}^{208}$ the underestimate can be as large as $0.055 \mathrm{fm}$. When this is taken into account, the predicted value of $S_{s} / S_{v}=2.0 \pm 0.3$ assuming $S_{v}=31$ $\mathrm{MeV}{ }^{17}$ Performing a series of Skyrme Hartree-Fock calculations of ${ }^{208} \mathrm{~Pb}$, in which values of $S_{v}$ and $L$ were systematically varied, Ref. 63] established that

$$
\begin{aligned}
\frac{r_{n p}}{\mathrm{fm}} & \simeq-0.094669+\frac{7.2028 S_{v}}{\mathrm{GeV}}+\frac{2.3107 L}{\mathrm{GeV}} \\
& -\frac{8.8453 S_{v}^{2}}{\mathrm{GeV}^{2}}-\frac{47.837 S_{v} L}{\mathrm{GeV}^{2}}+\frac{4.003 L^{2}}{\mathrm{GeV}^{2}}
\end{aligned}
$$

This formula, with the aforementioned value for $r_{n p}$, establishes the correlation slope $d L / d S_{v} \simeq-4.6$, assuming $S_{v}=31 \mathrm{MeV}$ and $L=45 \mathrm{MeV}$. More work is necessary to check the model dependence of this result, although it is in essential agreement with the liquid droplet prediction.

The constraint for the electric dipole polarizability $\alpha_{D}$ of ${ }^{208} \mathrm{~Pb}$ is taken from data produced by Tamii et al. [79]: $\alpha_{D} \simeq(20.1 \pm 0.6) \mathrm{fm}^{3}$. The liquid droplet prediction, Eq. 62, is $S_{s} / S_{v}=1.09 \pm 0.14$ assuming $S_{v}=31 \mathrm{MeV}$, which is slightly inconsistent with the neutron skin prediction. Roca-Maza et al. 80] showed from studies with a series of relativistic and non-relativistic interactions

\footnotetext{
${ }^{17}$ Danielewicz \& Lee [73] compiled a different set of ${ }^{208} \mathrm{~Pb}$ neutron skin thickness measurements 66 74, 75, 76, 77. to establish a weighted average $r_{n p}=(0.179 \pm 0.023)$ fm, just 0.004 fm larger than determined by Ref. 63]. Ref. 69] gives $0.16 \pm 0.02$ and a more recent study 78 found $r_{n p}=0.161 \pm 0.042 \mathrm{fm}$ for ${ }^{208} \mathrm{~Pb}$. The latter yields $S_{s} / S_{v}=1.7 \pm 0.6$ when diffuseness corrections are applied.
} 
that $\alpha_{D}, S_{v}$ and $r_{n p}$ for ${ }^{208} \mathrm{~Pb}$ can be constrained by

$$
\alpha_{D} S_{v} \simeq(325 \pm 14)+(1799 \pm 70)\left(r_{n p} / \mathrm{fm}\right) \mathrm{Mev} \mathrm{fm}^{3} .
$$

By use of Equation 72 , this is converted onto the $S_{v}-L$ correlation shown in

\section{The High-Density EOS and Mass and Radius Constraints}

For densities above the core-crust transition density, we will utilize separate polytropic regions with continuous pressures at the boundaries. Each region

\footnotetext{
${ }^{18}$ The slope of this correlation is significantly different than shown in 39, which relied on the analysis in Ref. 81 that erroneously concluded $\alpha_{D} \propto r_{n p}$.

${ }^{19}$ In comparison to the consensus region found in Ref. [39], the region displayed in Fig. 6] is slightly smaller because of the incorporation of the additional constraint from isobaric analog states.
} 
475 scheme explored by Read et al. [84. They found that a scheme with only three parameters could match, to about $4 \%$ rms error, the pressure-energy density relations for a large set of candidate EOSs up to densities below the central densities of $1.4 M_{\odot}$ stars. With an additional parameter, more exotic EOSs with of the first polytrope is the core-crust transition density $n_{0}$ and the starting densities of the upper two polytropes are fixed at $1.85 n_{s}$ and $3.7 n_{s}$, respectively. The four parameters are the three polytropic indices and the pressure at the starting density of the second polytrope. A more general scheme would have 7 parameters, three parameters corresponding to boundary density points above the core-crust transition and four corresponding to the polytropic exponents in each region.

In the models we will consider, the boundaries are denoted $n_{i}$ with $i=0-3$, and the adiabatic exponents between the densities $n_{i}$ and $n_{i-1}$, for $i=1-3$, for densities $n>n_{3}$ matter is treated as a continuation of region 3 , so that $p=p_{2}\left(n / n_{2}\right)^{\gamma_{3}}{ }^{21}$ We assume values at the core-crust transition are given by Eq. 13 with $n_{0}=n_{s} / 2.7$ and that the EOS in region 1 matches neutron matter calculations.

\subsection{A First Model: Fixed Boundaries}

In our first model, we choose $n_{1}=1.85 n_{s}$ and $n_{2}=3.74 n_{s}$ to match the optimum transition densities found by Ref. [84. The three remaining free parameters are the polytropic exponents $\gamma_{i}$ for $i=1-3$. We choose as parameters,

\footnotetext{
${ }^{20}$ Alternatively, one could use a parameterization employing energy densities rather than baryon densities, and using $p_{i}=K_{i}\left(\epsilon / \epsilon_{i-1}\right)_{i}^{\gamma}$. It would produce no substantial difference to our conclusions.

${ }^{21}$ In the scheme of Read et al. 84, the density boundary $n_{3}$ is used to define $\gamma_{3}$, see Eq. (76), but is otherwise irrelevant because $\gamma_{3}$ applies for all densities $n>n_{2}$. Note that our notation is not identical to that of Ref. [84.
} 
instead, the boundary pressures $p_{i}$ for $i=1-3$, following Ref. [85], by taking $n_{3}=7.4 n_{s}$. The first boundary, $n_{1}$, is close to the upper range of validity of neutron matter calculations, so values for $p_{1}$ were evaluated for each neutron matter EOS using Eq. 222); results are given in Table 2. We restrict the allowed $p_{1}$ range to lie within these results. except that we will slightly expand the upper and lower limits. The polytropic exponents and the energy densities within each region are given, assuming continuity of both energies and pressures at the boundary points $n_{i}$, by

$$
\begin{aligned}
& n(p)=n_{i-1}\left(\frac{p}{p_{i-1}}\right)^{1 / \gamma_{i}}, \quad \gamma_{i}=\frac{\ln \left(p_{i} / p_{i-1}\right)}{\ln \left(n_{i} / n_{i-1}\right)} \\
& \epsilon(p)=\left(\epsilon_{i-1}-\frac{p_{i-1}}{\gamma_{i}-1}\right)\left(\frac{p}{p_{i-1}}\right)^{1 / \gamma_{i}}+\frac{p}{\gamma_{i}-1},
\end{aligned}
$$

where $\epsilon_{i-1}=n_{i-1}\left(m_{n}+E_{i-1}\right)$ and $E_{i-1}$ is energy density and energy per baryon at the point $n_{i-1}$. For $p>p_{3}$, the same expressions are used as for $p_{2}<p<p_{3}$.

In this scheme the sound speed increases monotonically with density, so that causality within each region requires

$$
\frac{c_{s, i}^{2}}{c^{2}}=\left(\frac{\partial p}{\partial \epsilon}\right)_{i}=\frac{\gamma_{i, \max } p_{i, \max }}{\epsilon_{i, \max }+p_{i, \max }} \leq 1,
$$

which is an implicit equation for $p_{i, \max }$ after using Eq. 767. In general, $p_{1, \max }$ is so much larger than the realistic range for $p_{1}$ established from neutron matter studie $2^{22}$ that this causality condition is not important to our studies. $p_{3, \max }$ depends upon $p_{2}$ and $\epsilon_{2}$, and therefore also upon $p_{1}$. However, its dependence upon $p_{1}$ is weak, as shown in Fig. 7. The central density of the star is, in many cases, larger than $n_{3}$, in which case the limiting value $p_{3, \max }$ is smaller than the limit given by Eq. (77). The actual limit must be found numerically from TOV integrations of the star's structure requiring that the maximum sound speed at the center of the maximum mass star be smaller than $c$.

\footnotetext{
${ }^{22} p_{1, \max } \simeq 113.9 \mathrm{MeV} \mathrm{fm}^{-3}$ from causality considerations, see Fig. 7
} 


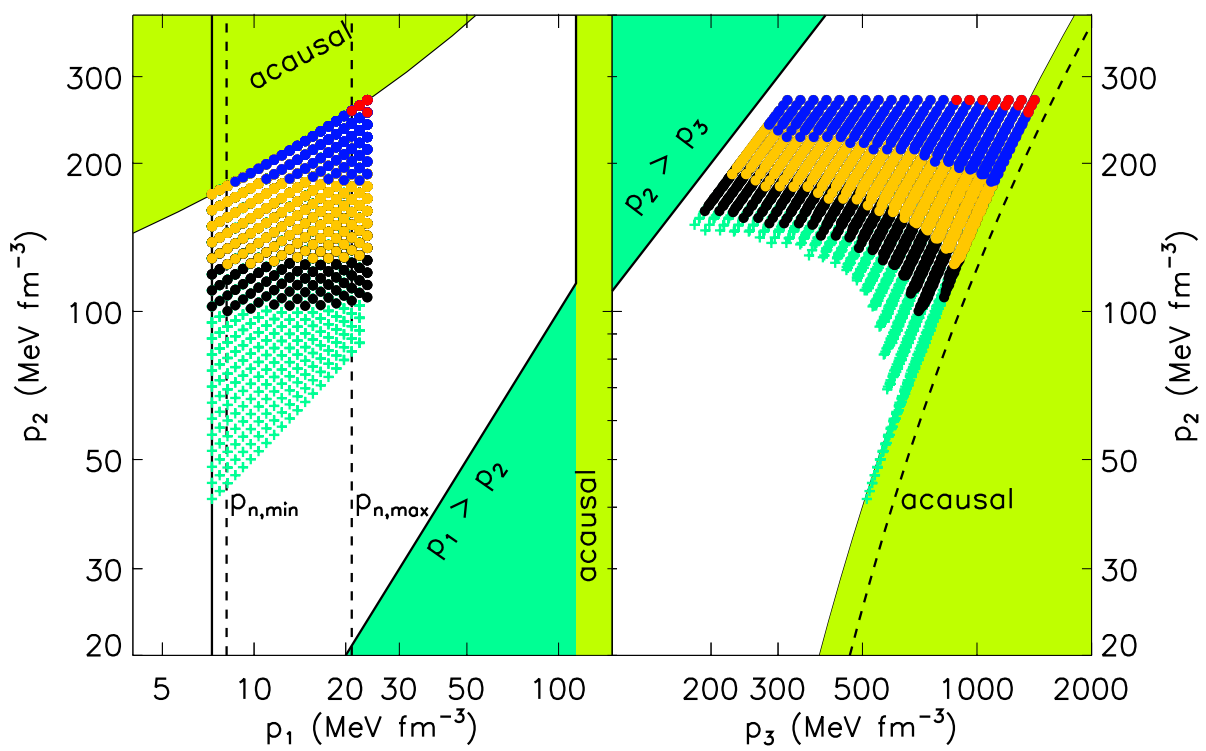

Figure 7: Permitted ranges of $p_{1}, p_{2}$ and $p_{3}$ from causality and stability. Values ruled out by causality (hydrodynamic instability) are indicated by the light-(dark-)green shading. Realistic minimum and maximum values of $p_{1}$ are shown as vertical dashed lines, and the value $p_{1, \min }=7.56 \mathrm{MeV} \mathrm{fm}^{-3}$ is the vertical solid line. Solid dots show parameter values permitting maximum masses, respectively, of $1.97 M_{\odot}$ (black), $2.10 M_{\odot}$ (orange), $2.30 M_{\odot}$ (blue) and $2.50 M_{\odot}$ (red). Teal crosses show parameter values that lead to acausal configurations or those having $M_{\max }<1.97 M_{\odot}$.

Minimum values for $p_{1}, p_{2}$ and/or $p_{3}$ also exist in order to satisfy hydrodynamic stability, which requires that $p_{i}>p_{i-1}$. Parameter ranges allowed by hydrodynamical stability and causality are portrayed in Fig. 7 as the white regions ${ }^{23}$ For $p_{2}$ and $p_{3}$, more restrictive minima can result if one requires that the maximum mass exceeds the largest well-measured neutron star mass, which we take to be $M_{\max }=1.97 M_{\odot}$, the $1 \sigma$ lower limit to the measured mass of PSR J0548+0432 [4]. (Note that there is no minimum value for $p_{1}$ based on this condition, due to the presence of the polytropic regions 2 and 3.) These limits must be found numerically from TOV integrations, which indicate that

${ }^{23}$ We note that the causality boundaries differ from those shown in Ref. 85. 
the effective lower limit to $p_{2}$ is approximately $100 \mathrm{MeV} \mathrm{fm}^{-3}$ for virtually all realistic choices of $p_{1}$ (Fig. 7).

The result of each TOV integration with a different EOS (i.e., different combinations of $p_{1}, p_{2}$ and $p_{3}$ ) is indicated by a symbol in Fig. 7 (many parameter combinations yield nearly identical configurations and cannot be distinguished). It is clear that causal configurations capable of supporting $M_{\max }=1.97 M_{\odot}$ must have $p_{2} \gtrsim 100 \mathrm{MeV} \mathrm{fm}^{-3}$, and if $M_{\max }=2.1 M_{\odot}, p_{2} \gtrsim 125 \mathrm{MeV} \mathrm{fm}^{-3}$. On the other hand, the specific value of $p_{3}$ plays relatively little role as long as $p_{2}<p_{3}<p_{3, \max }$ leads to causal configurations of the required mass.

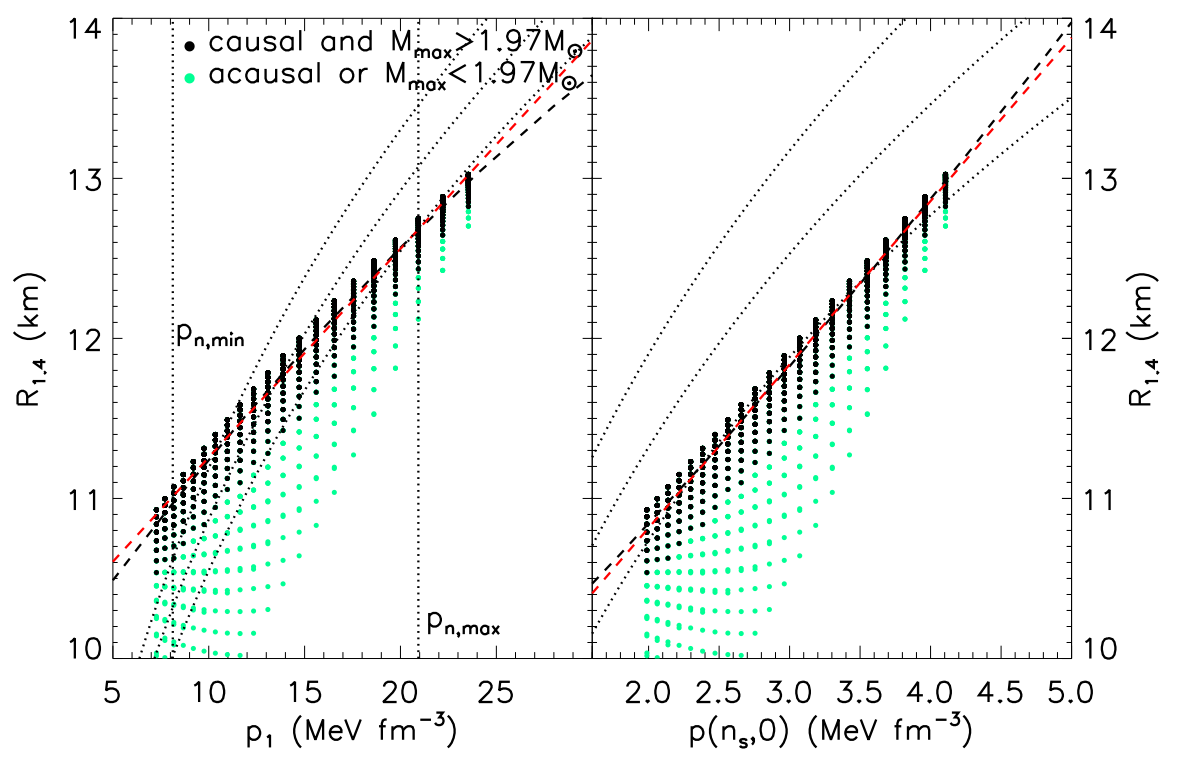

Figure 8: The correlations between radii of $1.4 M_{\odot}$ stars, $R_{1.4}$, and $p_{1}$ (left panel) and $p\left(n_{s}\right)$ (right panel). Parameters producing causal configurations capable of supporting $1.97 M_{\odot}$ are indicated as black circles; all others are indicated by teal circles. The solid (dashed) lines indicate quadratic (linear) fits to the black circles. The dotted curves show the correlations inferred from Eq. [3] [39] with $1 \sigma$ errors.

As expected, neutron star radii will be most sensitive to the parameter $p_{1}$. Values of $R_{1.4}$ for different choices of parameters are shown in Fig. 8 as functions of either $p_{1}$ or $p\left(n_{s}\right)=p_{1}\left(p\left(n_{s}\right) / p 1\right)^{\gamma_{1}}$. The spread of radius values for a given $p_{1}$ 
or $p\left(n_{s}\right)$ shows the influence of variations in $p_{2}$ and $p_{3}$ and is small. We compare the obtained correlations with the formulae earlier obtained by Ref. [39], Eq. (3) in Fig. 8. The radii obtained here tend to be somewhat smaller due to the ceiling placed on $p_{1}$ from neutron matter calculations.

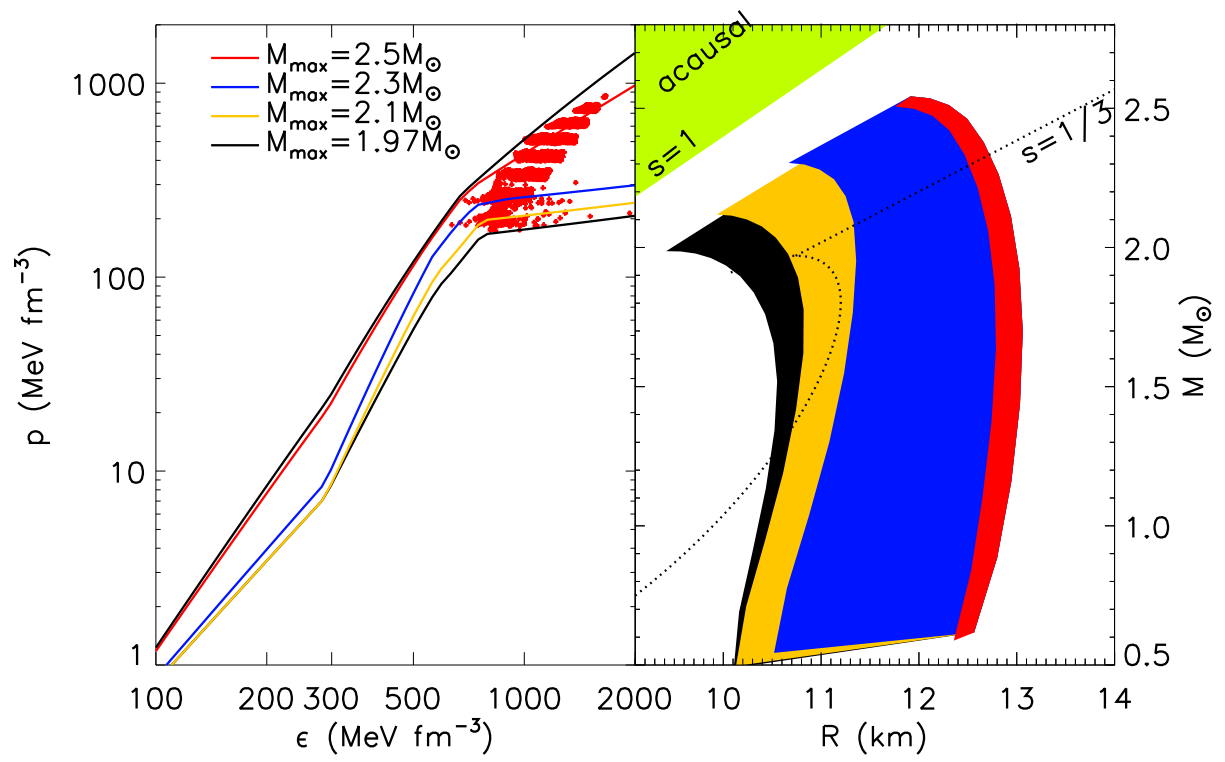

Figure 9: Left panel: Allowed pressures as a function of energy density permitted by the assumed constraints on the low-density EOS, causality, and selected values for $M_{\max }$. Red crosses indicate the central conditions for surviving EOSs. The black, yellow, blue and red lines are for $M_{\max }=1.97 M_{\odot}, 2.1 M_{\odot}, 2.3 M_{\odot}$, and $2.5 M_{\odot}$, respectively. Right panel: Allowed masses and radii for selected values of $M_{\max }$.

The restrictions of causality and large maximum masses severely restrict the allowed EOSs. The left panel of Fig. 9 shows boundaries in the pressureenergy density plane with different assumptions for $M_{\max }$ permitted by causality and the assumed low-density EOS for the crust and for neutron matter. For $M_{\max }=1.97 M_{\odot}$, the maximum uncertainty in pressure for a given energy density is no larger than a factor of 3 (which occurs near $n_{1}$ ), and is slightly larger than a factor of 2 near the central densities of maximum mass stars. The corresponding regions in the mass-radius plane that can be populated by EOSs 
satisfying the $M_{\max }$, causality and the low-density EOS constraints are also shown in this figure. Collectively, one observes the importance of neutron star mass measurements: the larger the minimum value of $M_{\max }$, the more restricted the ranges of $p(\epsilon)$ and $R(M)$ and the more accurately the EOS can be predicted.

The range $10.7 \mathrm{~km} \leq R_{1.4} \leq 13.1 \mathrm{~km}$ results from the restrictions we imposed 545 from neutron matter calculations and causality. This is of interest since many recent estimates of neutron star radii from observations have exceeded this range from both sides, as we will observe in Sec. 5 .

\subsection{A Second Model: Phase Transitions}

Lattimer \& Steiner [59] noted that phase transitions near $n_{s}$ have the effect of allowing both smaller and larger ranges of $R_{1.4}$. Similar results have been noted by Hebeler et al. [55, who reported $9.7 \mathrm{~km}<R_{1.4}<13.9 \mathrm{~km}$. However, the range is very sensitive to the density at which the phase transition is assumed to exist, and the widest range is observed when the phase transition begins at $n_{s}$. This seems an extreme lower limit, as there exists no experimental evidence for a phase transition in nuclei.

We explore the role of phase transitions by allowing the lower and upper transition densities, $n_{1}$ and $n_{2}$, respectively, to vary. We consider two models for matter with $n \geq n_{u}$, both described by $p=s \epsilon-\epsilon_{B}$ where $s$ is 1 or $1 / 3$ and $\epsilon_{B}$ is a constant related to the energy density where the pressure vanishes. The case $s=1$ specifies a phase transition to the stiffest EOS allowed by causality, while $s=1 / 3$ mimics a phase transition to a deconfined strange quark matter phase where $\epsilon_{B}=4 B / 3$ with $B$ the bag constant. These models are similar to the cases considered by Alford et al. [86.

The phase transition (assumed to be of first order here) is accomodated by requiring that $p_{\ell}=p_{u}$ and $\mu_{1}=\mu_{2}=\left(\epsilon_{1}+p_{1}\right) / n_{1}=\left(\epsilon_{2}+p_{2}\right) / n_{2}$ have the same values on both sides of the transition, $n_{1}$ being in the hadronic phase, and $n_{2}$ being in the high-density phase. For given values of $s$ and $\epsilon_{B}$, this uniquely 
specifies $n_{2}$ in terms of $n_{1}$ :

$$
n_{2}=\frac{(1+s) p_{1}\left(n_{1}\right)+\epsilon_{B}}{s \mu_{1}\left(n_{1}\right)} .
$$

The energy density changes by the amount $\epsilon_{2}-\epsilon_{1}=\mu\left(n_{2}-n_{1}\right)$ across the transition. Therefore, all possible phase transitions can be parameterized by $n_{1}, n_{2}$ and $s$. Results are shown in Fig. 10 for the two cases and are similar to those found by Ref. [86]. As expected, both the smallest and largest values of $R_{1.4}$ are found when $n_{1}=n_{s}$ and $s=1$. The largest values, about $14.3 \mathrm{~km}$, are found for $n_{2}=n_{1}$. The smallest values, about $8.4 \mathrm{~km}$, occur when $n_{2} \simeq 4.2 n_{1}$, and are nearly as small as that deduced from the maximally compact EOS for $M_{\max }=1.97 M_{\odot}(\simeq 8.1 \mathrm{~km}$ from Fig. 2). A phase transition is compatible with $M_{\max }=1.97 M_{\odot}$ only if the transition occurs close to $n_{s}$; in fact, $n_{1} \lesssim 2 n_{s}$ is required.

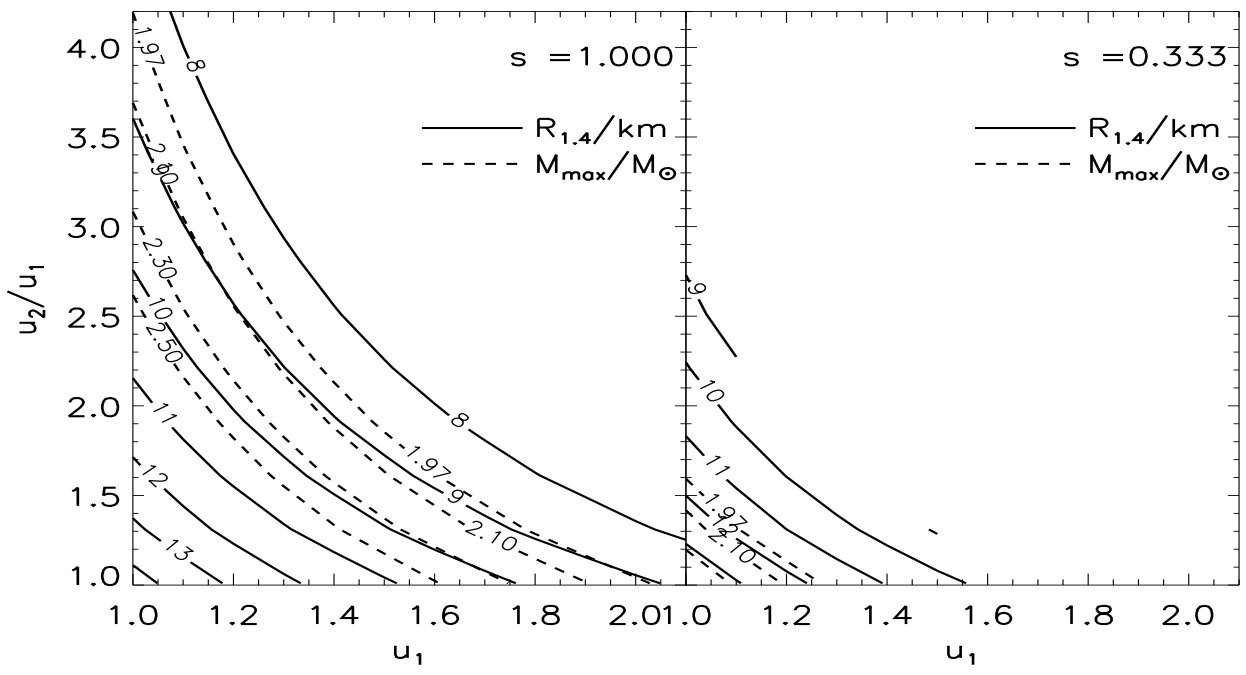

Figure 10: Radius limits for the case including phase transitions. Values of $R_{1.4}$ and $M_{\max }$ are displayed as functions of $u_{1}=n_{1} / n_{s}, u_{2}=n_{2} / n_{s}$ and $s$. The left (right) panel shows the case $s=1(s=1 / 3)$.

In the case that $s=1 / 3$, one sees from Fig. 10 that $11.7 \mathrm{~km}<R_{1.4}<13.8$ 575 km, which has an upper limit nearly the same as in Hebeler et al. [55] but a lower limit above what we established above. Moreover, as noted by Ref. 
[86, only a small range of values of $u_{1}$ and $u_{2}$ support a phase transition and $M_{\max } \gtrsim 1.97 M_{\odot}$. Using only observations of pulsar masses and estimates of symmetry properties from neutron matter studies and nuclear experiments, we can conclude, at present, that while the most extreme permitted range is 8.4 $\mathrm{km}<R_{1.4}<14.3 \mathrm{~km}$, a realistic range is about half as broad, being $10.7 \mathrm{~km}$ $<R_{1.4}<13.1 \mathrm{~km}$. It is interesting to compare these results with the radius values estimated from X-ray observations of neutron stars.

\section{Summary of Observational Constraints}

\subsection{Photospheric Radius Expansion Bursts}

Ref. 87] proposed using photospheric radius expansion (PRE) X-ray bursts to obtain simultaneous mass and radius measurements in 1979, but the method did not lead to interesting constraints until 2006 [88]. Type I X-ray bursts originate in accreting binaries when sufficient combustible mass accumulates onto a neutron star surface. The fuel ignites, with burning spreading quickly across the entire stellar surface. A subset of these bursts, PRE bursts are energetic enough to lift the stellar photosphere to great heights. This requires luminosities exceeding the so-called Eddington luminosity 24

$$
L_{E d d, \infty}=\frac{4 \pi c G M}{\kappa} \sqrt{1-\frac{2 G M}{R_{p h o t} c^{2}}},
$$

where $\kappa$ is the atmosphere's mean opacity and $R_{p h o t}$ is the distance of the photosphere from the star's center ${ }^{25}$ The bursts are short, lasting a matter of seconds, and the lifted material quickly loses opacity and falls back to the stellar surface. The burst decays as the stellar surface cools. Measurements of the flux and temperature during this decay permit the extraction of an angular area for the source

$$
A=\frac{F_{\infty}}{\sigma_{B} T_{c, \infty}^{4}}=\frac{1}{f_{c}^{4}}\left(\frac{R_{\infty}}{D}\right)^{2}
$$

\footnotetext{
${ }^{24}$ Quantities with the $\infty$ subscript are redshifted, as viewed at the Earth.

${ }^{25} R_{\text {phot }}>>$ during the first part of the burst, and in principle $R_{\text {phot }}=R$ when "touchdown" occurs and $L_{E d d}$ is measured.
} 
where the flux and color and effective temperatures at Earth are $F_{\infty}, T_{c, \infty}$ and $T_{e f f, \infty}$, respectively. $f_{c}=T_{e f f} / T_{c}$ is the color correction factor which accounts for the effects of a non-blackbody atmosphere, $R_{\infty}=R / \sqrt{1-2 G M /\left(R c^{2}\right)}$ is the apparent radiation radius and $D$ is the distance.

Quantities extracted from observations include $A, F_{E d d, \infty}=L_{E d d, \infty} /\left(4 \pi D^{2}\right)$, and $D$ (see Ref. [85] for a summary and references). Quantities estimated from theory are $\kappa$ and $f_{c}$. One can form two parameters from the observables:

$$
\alpha \equiv \frac{F_{E d d, \infty}}{\sqrt{A}} \frac{\kappa D}{f_{c}^{2} c^{3}}=\beta(1-2 \beta), \quad \gamma \equiv \frac{A}{F_{E d d, \infty}} \frac{f_{c}^{4} c^{3}}{\kappa}=\frac{R}{\beta(1-2 \beta)^{3 / 2}}
$$

The quantity $\gamma$ is independent of $D$, and $R_{\infty}=\alpha \gamma$ is independent of $\kappa$ and $F_{E d d, \infty}$. Note that $R \leq \sqrt{27 / 3125} \gamma$ since $\beta(1-2 \beta)^{3 / 2}$ has a maximum when $\beta=1 / 5$. The solutions for mass and radius become

$$
\beta=\frac{1}{4} \pm \frac{\sqrt{1-8 \alpha}}{4}, \quad R_{\infty}=\alpha \gamma
$$

$\alpha$ should be less than $1 / 8$ for real solutions to exist. In practice, however, observed values of $\alpha$ are greater than $1 / 8$ to more than $1 \sigma$ (Fig. 11). If one does Monte Carlo sampling within the error ranges of the observables, the resulting values of $\alpha$ and $\gamma$ mostly lead to complex solutions or acausal solutions (according to the maximally compact solution with $M_{\max }=1.97 M_{\odot}$ ). If one restricts acceptances to valid trials, $\alpha \approx 1 / 8$ becomes strongly preferred, leading to $\beta \approx 1 / 4$. Preferred values for $\gamma$ lie near their $1 \sigma$ upper limits. Values for $R_{\infty} \approx \gamma / 8$ then range from 11 to $14 \mathrm{~km}$, so that values for $R \approx R_{\infty} \sqrt{1 / 2}$ range from 8 to $10 \mathrm{~km}[89$. That only extremely small fractions (i.e., about 4\%) of trials are valid indicates serious problems with the model. The weighted means of the radii and masses of the sources are $10.6 \pm 0.7 \mathrm{~km}$ and $1.63 \pm 0.15 M_{\odot}{ }^{26}$

One possible solution to the small acceptance, suggested by Steiner et al. [89], is that the Eddington flux is measured while the photosphere is still well above the surface, so the redshift factor in Eq. 79 is absent. In this case, one

\footnotetext{
${ }^{26}$ This radius is about $1 \mathrm{~km}$ larger than in previous studies with this method, cf. Refs. 90. 91, due to the failure to impose causality on solutions.
} 


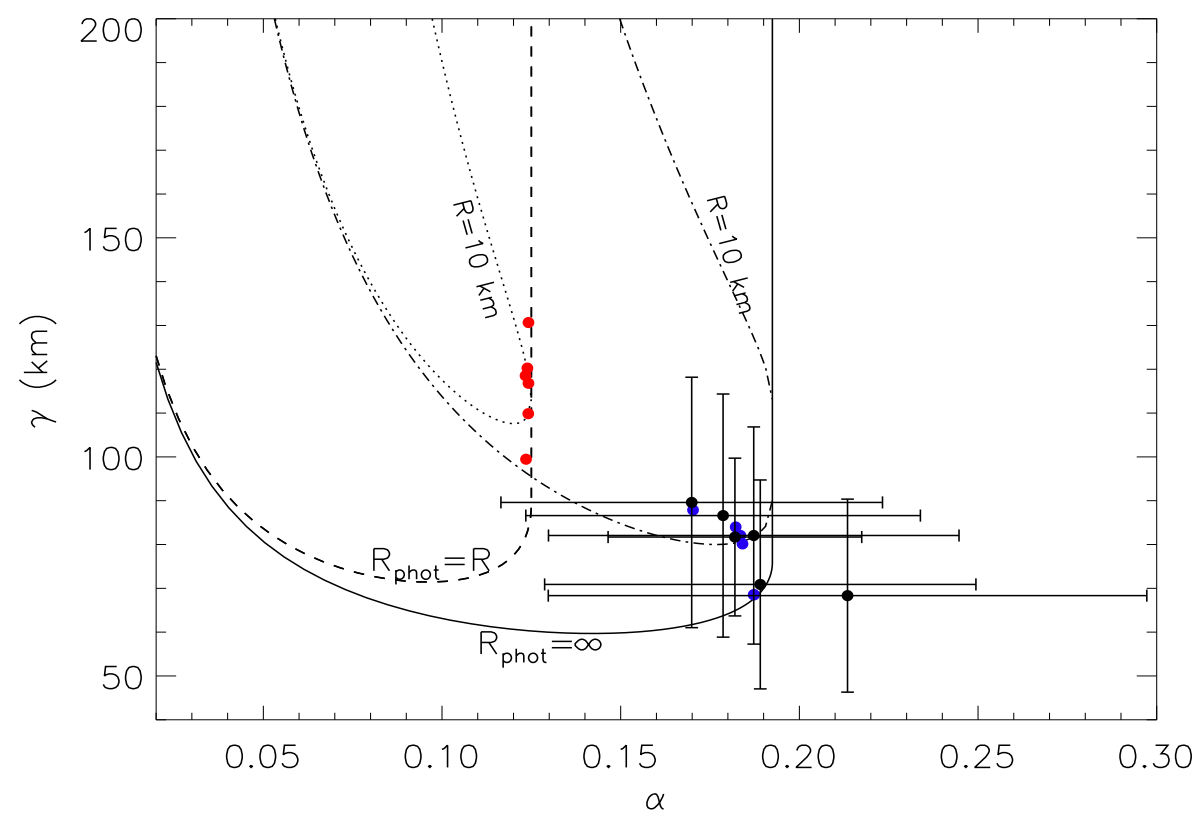

Figure 11: Allowed regions in $\alpha-\gamma$ space for real and causal solutions from PRE bursts lie above the dashed (solid) boundary curve for the $R_{p h o t}=R\left(R_{p h o t}=\infty\right)$ model. The boundaries represent $M$ and $R$ from the maximally compact solution with $M_{\max }=1.97 M_{\odot}$ translated to $\alpha$ and $\gamma$ using Eq. 82 [Eq. 83]. Data (black filled circles) and $1 \sigma$ errors for PRE burst sources are taken from Ref. 85. The dotted (dot-dashed) curve is the contour $R=10 \mathrm{~km}$ in the $R_{\text {phot }}=R\left(R_{\text {phot }}=\infty\right)$ model. The red (blue) filled circles show the maximum of the Monte Carlo distributions for the $R_{\text {phot }}=R\left(R_{\text {phot }}=\infty\right)$ model.

finds

$$
\alpha=\beta \sqrt{1-2 \beta}, \quad \gamma=\frac{R}{\beta(1-2 \beta)},
$$

whose real solution requires $\alpha \leq 3^{-3 / 2} \simeq 0.192$. All PRE burst sources shown in Fig. 11 satisfy this to within $1 \sigma$. As a result, a much larger trial acceptance rate ensues, about $65 \%$, after ensuring causality with $M_{\max }=1.97 M_{\odot}$. Note now that $R \leq \gamma / 8$, a factor 1.34 times larger than for the previous model. The weighted means of the radii and masses of the sources become $(11.2 \pm 0.7) \mathrm{km}$ and $(1.59 \pm 0.13) M_{\odot}$. The mean radius agrees to within $1 \sigma$ with those predicted from nuclear experiment and theory, and the mean mass agrees with the observed pulsar mass distribution. Nevertheless, the actual radius at touch- 
down is but one example of systematic uncertainties that arise in this simple description of PRE bursts. Ref. 92] used longer bursts and a more complete model of the neutron star atmosphere to obtain a radius greater than $14 \mathrm{~km}$ for the single source studied. The possible obscuration of a large fraction of the burst luminosity by the accretion disc was suggested as an important systematic uncertainty as well.

615

Another approach is to limit the study to those bursts in which one can avoid the uncertainty of the location of the photospheric radius, i.e., choosing only "passive" bursts, and also using information from the whole cooling tail rather than just near touchdown and at late times. Nättila et al. 93 recently performed a study of three sources with this cooling tail method and showed that the parameter $F_{E d d, \infty}$ could be determined to at least $3 \%$ accuracy and $A^{\prime}=D / R_{\infty}=\left(f_{c}^{4} A\right)^{-1 / 2}$ to about $1 \%$. One finds that the parameters $\alpha$ and $\gamma$ from Eq. (81) for the three sources each have nearly the same central values: $\alpha \simeq 0.117$ and $\gamma \simeq 128 \mathrm{~km}$ to about $2 \%$. The major uncertainty in $\alpha$ is due to the roughly $10 \%$ distance uncertainty, while $\gamma$ has less than $4 \%$ uncertainty. Since $\alpha$ is smaller than $1 / 8$ to about $1 \sigma$, most solutions from parameters taken within the error distributions will be real. From Eq. 82, $\beta \simeq 0.185$ or 0.315 and $R_{\infty} \simeq 15 \mathrm{~km}$ (all are central values). The centroids of the two solutions thus have $M=1.5 M_{\odot}$ and $R=11.9 \mathrm{~km}$, and $M=1.94 M_{\odot}$ and $R=9.1 \mathrm{~km}$, respectively. The second solution can be rejected on the basis of its incompatibility with the symmetry energy of nuclear matter; it is also extremely close to the acausal boundary in $M-R$ space with $M_{\max }=1.97 M_{\odot}$ (Fig. 3). In any case, the small uncertainty in $\gamma$ and its value requires that $R<12 \mathrm{~km}$ for any value of $\alpha$.

\subsection{Quiescent Low-Mass X-Ray Binaries}

635

Another popular method of measuring neutron star radii using observations of thermal emission utilizes accreting neutron stars in binary systems, known as quiescent low-mass X-ray binaries (qLMXBs). The earliest such observations led to blackbody radius estimates of less than $1 \mathrm{~km}$, much smaller than 
that predicted from theoretical models 94]. For accreting neutron stars, the photosphere is expected to consist of hydrogen [95], and Ref. [96] showed that models of thermal emission from pure hydrogen photospheres [97, 98, give inferred radii of the correct order of magnitude. Continued observations and a consistent treatment of the surface gravity [99, 100] led to refined estimates of radii between 8 and $16 \mathrm{~km}$ for sources in globular clusters where $D$ is reasonably well known. However, by themselves, this result cannot rule out a significant number of EOS models 38.

Guillot et al. [101] combined new qLMXB observations and a "common radius" model (motivated by the generic vertical shape of $M-R$ curves, cf. Fig. 2 to obtain a rather small preferred neutron star radius with a corresponding 650 small error, $9.0 \pm 1.4 \mathrm{~km}$. However, when individually analyzed, the sources predicted radii in a wide range $(7$ to $20 \mathrm{~km})$. Ref. [102 concluded that two systematic uncertainties were responsible for this result: (i) some of the smaller radius neutron stars may have helium rather than hydrogen atmospheres, and (ii) the estimated amount of X-ray absorbing matter between the sources and Earth might be wrong. Ref. 103] showed that a helium (rather than hydrogen) atmosphere changes the inferred radius range for the neutron star in M28 from 6 to $11.5 \mathrm{~km}$ to 7 to $17 \mathrm{~km}$. Heinke et al. [104 confirmed that the absorbing mass inferred for the qLMXB with the largest inferred radius was indeed overestimated, leading to a systematic radius reduction. Ref. 104 also showed that the choice of the galactic abundance model was an important systematic affecting all sources.

A consequence of the common radius model is the tendency to underpredict the radius. Measurements of qLMXB spectra are better able to constrain $R_{\infty}$ than the surface gravity $g=G M /\left[R^{2} \sqrt{1-2 \beta}\right]$ of a source, since the main global observables are $F_{\infty}, T_{c, \infty}$ and $D$, and for a blackbody

$$
F_{\infty}=\left(\frac{R_{\infty}}{D}\right)^{2} \sigma_{B} T_{c, \infty}^{4}
$$

Corrections for the atmosphere, most often a low-magnetic field, pure $\mathrm{H}$ atmosphere, are readily produced (i.e., through $f_{c}$ ). A variable in $M$ and $R$ 
orthogonal to $R_{\infty}$, such as the surface gravity, is poorly constrained by available data for every source. Therefore, simultaneously refitting all sources under the constraint of a common radius is accommodated most easily by variations in $g$ rather than $R_{\infty}$. As is clear from the $R_{\infty}$ contours seen in Fig. 2 sources with widely varying estimated values of $R_{\infty}$ can satisfy the constraint of a common radius only if that radius is smaller than the smallest $R_{\infty}$ in the sample. Sources with large $R_{\infty}$ can be reconciled by increasing their mass (and $g$ ). For the analysis of Guillot et al. [101, the mean $R_{\infty}$ of the individually analyzed sources was $14.0 \mathrm{~km}$; for the joint analysis with a common radius, it became $13.2 \mathrm{~km}$. The predicted masses of the individually analyzed sources ranged over $0.8 M_{\odot}<M<1.8 M_{\odot}$; for the joint analyses the range increased to ${ }_{675} \quad 0.7 M_{\odot}<M<2.3 M_{\odot}$.

\subsection{Bayesian Analyses of Observations with Theoretical Priors}

Steiner et al. [89] used Bayesian inference to combine mass and radius constraints from both qLMXBs and PRE X-ray bursts to obtain constraints on the EOS. This work used a nuclear physics-based parameterization for matter near the nuclear saturation density and piecewise polytropes at higher densities (in a slightly different form than that presented in Ref. [84]). The Bayesian inference-based method to obtain the EOS parameters is similar to the method developed in Ref. [90, except that Ref. [89] additionally obtained probability distributions for the $M-R$ curve by marginalizing over the posteriors for $R$ as a function of $M$. Also, their direct use of Bayesian inference allowed the use of more parameters capable of exploring the uncertainties in the EOS near saturation densities and led to novel constraints on the density dependence of the nuclear symmetry energy.

Ref. [89] showed that the assumption that the photosphere at touchdown is coincident with the surface (i.e., $R_{p h o t}=R$ ) is not consistent with the data (within the context of the model being used for PRE X-ray bursts in Refs. 89] and [91]). This was confirmed with the introduction of Bayes factors in Ref. 102. Bayes factors also showed that helium, not hydrogen, is favored for at 
least one qLMXB source, which allows for larger radius estimates from these sources than found in Ref. [101]. The final result was a range of $R_{1.4}$ from 10.7 to $12.5 \mathrm{~km}$.

Ref. 93 performed a Bayesian analysis of the 3 sources they analyzed with the cooling tail method, using the same EOS and causality constraints as in Refs. [102, 105] and find, if strong phase transitions are disallowed, that 11.3 $\mathrm{km}<R_{1.4}<12.8 \mathrm{~km}$ to $95 \%$ confidence. Allowing strong phase transitions changes this range to $10.5 \mathrm{~km}<R_{1.4}<12.5 \mathrm{~km}$. All these ranges determined with the Bayesian method are tantalizingly similar to that predicted by neutron matter and nuclear experimental studies. However, reducing their uncertainty is still hostage to our lack of understanding of important systematics in the interpretation of observations.

\subsection{Other Observations}

We conclude this section with a brief survey of proposed radius measurements. These include measurements of the moment of inertia of the more massive pulsar in the pulsar binary PSR J0737-3039, pulse-profile observations of pulsars and bursting sources by X-ray telescopes to measure $z$ and $R$, and gravitational wave observations of merging compact objects: black hole-neutron star (BH-NS) and neutron star-neutron star (NS-NS) binaries.

\subsubsection{Moments of Inertia}

The moment of inertia is readily calculated in GR for the case of a uniformly, slowly rotating star. It can be expressed as

$$
I=\frac{c^{2}}{G} \frac{w(R) R^{3}}{6+2 w(R)},
$$

where $w(0)=0$ and $w(R)$ is the solution, at the surface $R$, of the differential equation

$$
\frac{d w}{d r}=\frac{4 \pi G}{c^{2}} \frac{(\epsilon+p)(4+w) r}{c^{2}-2 G m}-\frac{w}{r}(3+w),
$$

where $m(r)$ is the mass enclosed within radius $r$. 
The measurement of the moment of inertia from spin-orbit coupling in a compact binary is especially intriguiging since it is seemingly less subject to uncertain systematic effects than current methods. Spin-orbit coupling leads to geodetic precession of the angular momentum vector $\overrightarrow{\mathbf{L}}$ of the orbital plane around the direction of the total angular momentum $\overrightarrow{\mathbf{J}}$ of the system. Since the total angular momentum $\overrightarrow{\mathbf{J}}=\overrightarrow{\mathbf{L}}+\overrightarrow{\mathbf{S}_{\mathbf{A}}}+\overrightarrow{\mathbf{S}_{\mathbf{B}}}$ is conserved (at this order), there are compensating precessions of the spins $\overrightarrow{\mathbf{S}_{\mathbf{A}}}$ and $\overrightarrow{\mathbf{S}_{\mathbf{B}}}$ of the two stars. Since the orbital angular momentum dominates the spin angular momenta in binaries, the geodetic precession amplitude is very small, while the associated spin precession amplitudes are substantial. In addition to geodetic precession, spin-orbit coupling also manifests itself in apsidal motion (advance of the periastron).

According to Barker \& O'Connell [106], the spin and orbital angular momenta evolve as

$$
\begin{aligned}
\frac{d \vec{S}_{i}}{d t} & =\frac{G\left(4 M_{i}+3 M_{-i}\right)}{2 M_{i} a^{3} c^{2}\left(1-e^{2}\right)^{3 / 2}} \overrightarrow{\mathbf{L}} \times \overrightarrow{\mathbf{S}_{\mathbf{i}}}, \\
\frac{d \overrightarrow{\mathbf{L}}^{\mathrm{SO}}}{d t} & =\sum_{i} \frac{G\left(4 M_{i}+3 M_{-i}\right)}{2 M_{i} a^{3} c^{2}\left(1-e^{2}\right)^{3 / 2}}\left(\overrightarrow{\mathbf{S}_{\mathbf{i}}}-3 \frac{\overrightarrow{\mathbf{L}} \cdot \overrightarrow{\mathbf{S}_{\mathbf{i}}}}{|\overrightarrow{\mathbf{L}}|^{2}}\right),
\end{aligned}
$$

where the superscript "SO" refers to the spin-coupling contribution only (there are also first- and second-order post-Newtonian terms, unrelated to the spins, that contribute to this order). Here $a$ is the semi-major axis, $e$ is the eccentricity and $M_{i}$ is component $i$ 's mass. Note that if the spins are parallel to $\overrightarrow{\mathbf{L}}$ there is no precession. The change in inclination, due to changes in $\overrightarrow{\mathbf{L}}$, is $[107$.

$$
\frac{d i}{d t}=\frac{G}{a c^{2}} \frac{\pi}{\left(1-e^{2}\right)^{3 / 2}} \sum_{i} \frac{I_{i}\left(4 M_{i}+3 M_{-i}\right)}{M_{i} a^{2} P_{i}} \sin \theta_{i} \cos \phi_{i}
$$

where $\theta_{i}$ is the angle between $\overrightarrow{\mathbf{S}}_{i}, I_{i}$ is the moment of inertia of pulsar $i$, and $\overrightarrow{\mathbf{L}}$ and $\phi_{i}$ is a projection angle between the line of sight and $\overrightarrow{\mathbf{S}}_{i}$. The amplitude of pulsar's $i$ precession is $\delta_{i}=\left(\left|\overrightarrow{\mathbf{S}}_{i}\right| /|\overrightarrow{\mathbf{L}}|\right) \sin \theta_{i}$. Also $\left|\overrightarrow{\mathbf{S}}_{i}\right|=2 \pi I_{i} / P_{i}$ where $P_{i}$ is pulsar $i$ 's spin period. The periodic departure of pulse arrivals has an amplitude

$$
\delta t_{A}=\frac{M_{B}}{M} \frac{a}{c} \delta_{A} \cos i \simeq(1.7 \pm 1.6) I_{A, 80} \mu \mathrm{s},
$$

where $M$ is the total mass. We evaluated this for the case of pulsar PSR J07373039 and it is clear it is essentially unobservable since the system is nearly 


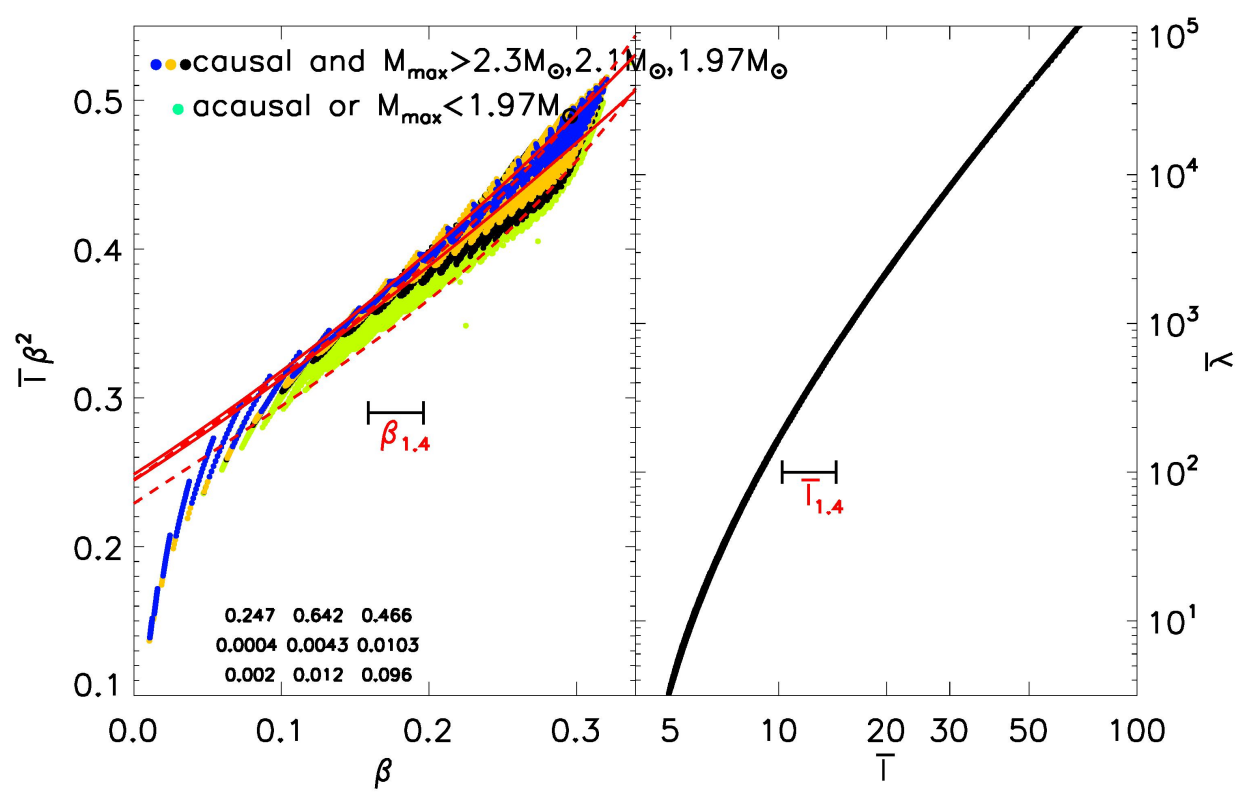

Figure 12: Quasi-universal behaviors of the moment of inertia with respect to $\beta$ ( left panel) and tidal deformability $(\bar{\lambda})$, right panel) of neutron stars. Note that $I \beta^{2} / M^{3} \propto I /\left(M R^{2}\right), \bar{I}=$ $I c^{4} /\left(G^{2} M^{3}\right)$ and $\bar{\lambda}=\lambda G^{4} /\left(M c^{2}\right)^{5}$. Each point is a stellar model computed with a piecewise polytropic EOS as described in Sec. 4.1 Models violating causality or not supporting $M_{\max }=$ $1.97 M_{\odot}$ are indicated by green points; other colors show causal results for various maximum masses. The compactness range $\beta_{1.4}$ for $1.4 M_{\odot}$ stars is indicated. Solid and dashed lines in the left panel indicate Eqs. 93) and 92, respectively. Adapted from Ref. 105.

On the other hand, the advance of the periastron is proportional to $I_{i}$ and its observability is maximized when $i=\pi / 2$, i.e., for edge-on orbits. It's observability is likely because it has a magnitude similar to the 2 nd post-Newtonian 
correction to periastron advance ${ }^{27}$

$$
\begin{aligned}
\frac{A_{p}}{A_{2 P N}} & \simeq-\frac{8 P c^{2}}{G\left(1-e^{2}\right)^{1 / 2} M^{2} a}\left(\frac{189}{1-e^{2}}-47\right)^{-1} \sum_{i} \frac{I_{i}\left(4 M_{i}+3 M_{-i}\right)}{M_{i} P_{i}} \cos \theta_{i} \\
& \simeq-1.30_{-0.12}^{+0.04} I_{A, 80} .
\end{aligned}
$$

It has been suggested [108, that a measurement accuracy of $10 \%$ is sufficient to place strong constraints on the EOS. In a recent unpublished talk in Montreal in 2015, P. Freire argued that systematics are in our favor in the case of PSR J07373039 in that a near cancellation of certain effects is occuring. The implication is that a measurement accurate to $10 \%$ is perhaps a decade away. To illustrate its usefulness, we note that nearly universal, i.e., EOS-independent, correlations between the moment of inertia and the stellar compactness exist (Fig. 12). Such a quasi-universal relation was quantified by Ref. [108]:

$$
I \simeq M R^{2}(0.237 \pm 0.008)\left[1+2.844 \beta+18.91 \beta^{4}\right]
$$

An improved fit, limited to $\beta \geq 0.1$ and based on an array of piecewise polytrope EOSs as described in Sec. 4.1 with $M_{\max } \gtrsim 1.97 M_{\odot}$, is 105.

$$
I \simeq M R^{2}\left[0.247 \pm 0.002+(0.642 \pm 0.012) \beta+(0.466 \pm 0.096) \beta^{2}\right]
$$

The masses in the PSR J0737-3039 system are known to high precision. The uncertainty in the fitting coefficients is sufficiently small that a $10 \%$ error in the measurement of $I$ will dominate the uncertainty $(\sim 6-7 \%)$ of a radius measurement.

\subsubsection{Pulse-profile observations}

Surface emission from spinning neutron stars produces a periodic brightness change as hot and cold spots move in and out of the line of sight. Brightness variations could be due to magnetic fields, by non-uniform thermonuclear burning in an X-ray burster, or anisotropic accretion from a companion. The strong surface

\footnotetext{
${ }^{27} \theta_{A} \simeq 13^{\circ}$ in PSR J0737-3039, so a small term from Ref. 108 is consequently ignored.
} 
gravity of a neutron star affects the pulse shapes and amplitudes; generally, the more compact the star, the more of its surface is visible to a distant observer, and the smaller the amplitudes. Fortunately, using pulse profiles to measure compacness does not depend on the star's distance or intervening absorbing matter, unlike measurements from PRE bursts or qLMXBs. The method is one motivation for the funded NASA's NICER (Neutron star Internal Composition ExploreR) [109 and the proposed ESO LOFT (Large Observatory For x-ray Timing) 110 missions.

Early attempts to employ this technique generally studied bolometric emissions [111, 112, 113, 114] which could not untangle degeneracies between $M$ and $R$ and produced estimated values for $\beta$ that, while consistent with neutron stars, lacked enough accuracy to be useful. It has been suggested, however, that pulse profiles observed in two or more energy bands supply additional constraints that can break these degeneracies [115, 116, 117, 118. The method makes use of nearly universal relations, similar to the correlations shown in Fig. 12, among properties of rotating neutron stars. These include the surface ellipticity, the specific angular momentum $a=I c /\left(G M^{2} P_{s}\right)$, where $P_{s}$ is the spin period, the star's quadrupole moment, $M$, and $R_{e q}$, the equatorial radius [119, 120, 121]. Models [117] show that the pulse profile is nearly independent of the angular size of a spot [113], and, using the aforementioned semi-universal relations, the profiles are determined essentially by $M, R_{e q}$, the inclination $i$, and $\theta_{s}$, the colatitude of the spot. $M, R_{e q}$ and $P_{s}$ are sufficient to determine $R$, the non-rotating radius for the same mass $M$, and, hence, will predict the EOS. The NICER mission was designed to acquire $M$ and $R$ to about $5 \%$ accuracy from at least one pulsing source, PSR J0437-4715.

\subsubsection{Gravitational wave observations}

The merger of compact binary stars is expected to be the main source of gravitational radiation signals observed with Advanced LIGO and VIRGO [122]. Detection of a regular sinusoidal waveform prior to the merger should allow determination of the so-called chirp mass $\mathcal{M}=\left(M_{1} M_{2}\right)^{3 / 5} / M^{1 / 5}$ to high accu- 
racy. Tidal effects during inspiral allow the possibility of breaking the remaining mass degeneracy [123] through the measurement of the tidal deformability $\lambda=2 k_{2} R^{5} /(3 G)$, where $k_{2}$ is the tidal Love number. The Love number, like the moment of inertia, can be found from the solution of a first-order differential equation [124.

$$
\begin{aligned}
\frac{d y}{d r} & =-\frac{y^{2}}{r}-\frac{y-6}{r-2 G m / c^{2}}-r Q, \\
Q & =\frac{4 \pi G}{c^{4}} \frac{(5-y) \epsilon+(9+y) p+(\epsilon+p) / c_{s}^{2}}{1-2 G m / c^{2}}-\left[\frac{2 G\left(m+4 \pi p r^{3} / c^{2}\right)}{r\left(r c^{2}-2 G m\right)}\right]^{2}, \\
k_{2} & =\frac{8}{5} \beta^{5}(1-2 \beta)^{2}\left[2-y_{R}+2 \beta\left(y_{R}-1\right)\right] / \mathcal{R} \\
\mathcal{R} & =6 \beta\left(2-y_{R}+\beta\left(5 y_{R}-8\right)\right)+4 \beta^{3}\left[13-11 y_{R}+\beta\left(3 y_{R}-2\right)+2 \beta^{2}\left(1+y_{R}\right)\right] \\
& +3(1-2 \beta)^{2}\left[2-y_{R}+2 \beta\left(y_{R}-1\right)\right] \ln (1-2 \beta),
\end{aligned}
$$

where $y(0)=2$ and $y_{R}=y(R)$.

The seemingly chaotic signal immediately following tidal disruption, interestingly, seems to have characteristics that depend primarily on the neutron star's tidal deformability. For black hole-neutron star (BH-NS) mergers, it has been argued that combining pre-merger and post-merger information best fits the tidal deformability, compared to other neutron star properties [125, 126]. For a neutron star-neutron star merger, the gravitational wave frequency at the peak of the signal (when tidal disruption occurs) has been found to strongly correlate with $\bar{\lambda}$ [127]. There are two dominant peaks in the gravitational wave spectrum of the postmerger phase in a neutron star-neutron star merger, which originates from fundamental quadrupolar oscillation modes, and lie in the range of $2-3.5 \mathrm{kHz}$. The lower frequency appears to correlate with the compactness $\beta$ in a quasi-universal relation [128. The higher frequency correlates with the radius $R_{1.6}$ of $1.6 M_{\odot}$ stars [129], but the relation is mass dependent. Takami et al. 130] instead argue that the higher frequency nicely correlates with the tidal deformability $\bar{\lambda}$. Thus, at least for neutron star mergers where masses can be sufficiently well determined, there are three separate possible constraints. While a single merger may not provide accurate enough information to determine $M$ 
and $R$ precisely, observations of several mergers may allow strong statistical information to be derived. This is especially true, given the extremely strong universal correlation between the tidal polarizability and the moment of inertia 785 (Fig. 12) 120.

\subsubsection{Rotation Periods}

Yet another nearly universal relation holds between the maximum spin frequency of a uniformly rotating neutron star and its average density [131, 132.

$$
f_{K}=1.08\left(\frac{M}{M_{\odot}}\right)^{1 / 2}\left(\frac{10 \mathrm{~km}}{R}\right)^{3 / 2} \mathrm{kHz},
$$

where $f_{K}$ indicates the Keperlian frequency at which mass shedding occurs. The $M$ and $R$ in this formula do not refer to the mass and radius of the spinning configuration but rather to the non-spinning star. Although this formula was phenomenologically established from full axially-symmetric solutions of Einstein's equations, the dependence on mass and radius is the same as in Newtonian gravity. Furthermore, the coefficient $1.08 \mathrm{kHz}$ is very close to the prediction of the analytic relativistic Roche model (which assumes the gravitational potential of the spinning star is given by concentrating all its mass at the origin) 133, 134, $\simeq 1.00 \mathrm{kHz}$. It is also interesting that the equatorial radius of the maximally rotating star is about 1.44 times $R$, the non-spinning radius for a star of the same mass $M$, compared to the analytic result of 1.5 from the relativistic Roche model. The highest frequency pulsar known is PSR J1748-2446ad [135] with $f=716 \mathrm{~Hz}$, and its limiting radius as a function of mass is shown in Fig. 2 Unfortunately, the mass of this pulsar is unknown, so an upper limit to its radius is unable to be established. However, if most rapidly spinning pulsars, so-called millisecond pulsars, acquired their angular momentum by accretion from a companion, it is not out of the question that a rapidly rotating millisecond pulsar will eventually be found in a binary system with measured masses. Assuming that $M \lesssim 2 M_{\odot}$, Eq. 95 implies $R \lesssim 16.6 \mathrm{~km}$. 


\subsubsection{Binding Energies}

The neutron star binding energy is the difference in mass between a star's matter if at infinite dispersion (the baryon mass $\mathcal{N} m_{b}$ ) and the gravitational mass $M$ :

$$
\mathrm{BE}=\mathcal{N} m_{b}-M=\int \frac{n m_{b} d^{3} r}{\sqrt{1-2 G m /\left(r c^{2}\right)}}-\int \epsilon d^{3} r .
$$

Its utility rests on quasi-universal relations connecting the binding energy to the compactness $\beta$ (cf. Ref. [38])

$$
\frac{\mathrm{BE}}{M c^{2}} \simeq(0.60 \pm 0.06) \frac{\beta}{1-\beta / 2}
$$

or to the moment of inertia $I$. An improved fit, based on an array of piecewise polytrope EOSs with $M_{\max } \gtrsim 1.97 M_{\odot}$ and limited to $\beta \gtrsim 0.1$, is [105,

$$
\mathrm{BE} \simeq \beta M c^{2}[0.564 \pm 0.010+(0.521 \pm 0.077) \beta] .
$$

An accurate determination could in principle be made from the neutrino signal from a supernova, in which the detector serves, essentially, as a calorimeter. Radius determinations would depend on the accuracy with which the mass of the remnant can be determined. Measurements of neutrinos from SN 1987A yielded a value in qualitative agreement with a neutron star of $1.2-1.5 M_{\odot}$ and a moderate radius, $\mathrm{BE} \approx(2-3) \cdot 10^{53}$ ergs. A contemporary supernova observation of a galactic supernova is likely to yield not a dozen events, as in the case of SN 1987A, but tens of thousands of events and a substantial statistical improvement.

Another suggested measure of binding energy is related to the lightest pulsar masses measured in neutron star binaries. Under the assumption that nearly no accretion has accumulated onto a non-recycled pulsar, theoretical estimates for the baryon mass of the remnant based on stellar evolution can be compared to the measured mass [136]. The lightest neutron stars may be formed by electroncapture supernovae, for which theoretical models imply a well-defined baryon mass $\mathcal{N} m_{b} \simeq(1.370 \pm 0.005) M_{\odot}$. As one example, the mass of pulsar $\mathrm{B}$ in the system PSR J0737-3039 is $(1.2489 \pm 0.0007) M_{\odot}$ [137] and is thought to 
have been formed in an electron-capture supernova. The difference between its present mass and the mass before collapse $\left(\simeq \mathcal{N} m_{b}\right)$, about $(0.121 \pm 0.005) M_{\odot} c^{2}$, represents its BE. For the case at hand, using Eq. [98), $\beta \simeq(0.154 \pm 0.008)$ and $R \simeq(12.0 \pm 0.6) \mathrm{km}$. However, if fall-back of matter onto the neutron star after the supernova occurs is significant, i.e., is more than $0.01 M_{\odot}$, the $\mathrm{BE}$ is undererestimated and $R$ is overestimated.

In summary, the future appears bright with prospects for further constraining neutron star radii and the dense matter EOS. We now turn to investigations of the thermal properties of dense matter.

\section{THERMAL EFFECTS IN BULK MATTER}

In homogeneous bulk matter comprised of nucleons (phase III in Fig. 1), chemical potentials, specific heats, etc., play important roles in the evolution of compact objects. Except under circumstances when matter is in the degenerate ( $T$ small compared to the Fermi temperature $T_{F}$ ) or the non-degenerate $\left(T \gg T_{F}\right)$ region, a transparent understanding of what governs the thermal analytically to yield closed-form expressions. Because of the varying concentrations of fermions (neutrons, protons and leptons) and bosons (photons, possible Bose condensates at supra-nuclear densities) encountered, one or the other constituent will likely lie in different regions of degeneracy. The limiting cases besides serving as consistency checks on numerical calculations of the thermal variables, and are discussed below.

\subsection{Non-degenerate Limit}

When the fugacities $e^{\mu_{i} / T}$ are small ( $\mu_{i}$ being the chemical potential of species $i$ ), non-degenerate (or dilute gas) conditions prevail. In this case, corrections to the ideal gas expressions for the thermal state variables are generally 
obtained by using the fugacity expansion or the virial expansion approach in which measured phase shifts serve as an input. For contact interactions, nonrelativistic Skyrme models being a prototype example, the thermal pressure $P_{t h}$ and energy density $\epsilon_{t h}$ for a single species of fermions can be written in terms of their ideal gas counterparts (calculated with the Landau effective mass $m^{*}(n)$ instead of $\left.m_{b}\right)$ as 138 ]

$$
\begin{aligned}
& P_{t h}(n, T)=P_{t h}^{i d}\left(n, T ; m^{*}\right)\left(1-\frac{3}{2} \frac{n}{m^{*}} \frac{d m^{*}}{d n}\right) \\
& \varepsilon_{t h}(n, T)=\varepsilon_{t h}^{i d}\left(n, T ; m^{*}\right), \quad P_{t h}^{i d} / \varepsilon_{t h}^{i d}=2 / 3,
\end{aligned}
$$

where $m^{*}=p_{F}(\partial \epsilon(p) / \partial p)_{p_{F}}^{-1}$ with $p_{F}$ denoting the Fermi momentum and $\epsilon(p)$ the single-particle spectrum. These results are valid for all regions of degeneracy and help to develop simple expressions in the non-degenerate (and degenerate) conditions (see e.g., Ref. [25]). For non-relativistic models with finite-range interactions, a method involving next-to-leading order steepest descent approach for the calculation of all the state variables has been developed in Ref. 23]. For relativistic mean-field theoretical (MFT) models, the appropriate formulas in the dilute gas limit can be found in Ref. [138.

\subsection{Degenerate Limit}

For nucleons in the homogeneous phase, Landau's Fermi Liquid Theory (FLT) provides simple analytical expressions that are model independent for the thermal state variables in the limit of extreme degeneracy $\left(T / T_{F} \ll 1, T_{F}\right.$ being the Fermi temperature) [139. In the absence of collective excitations close to the Fermi surface, thermal effects are primarily determined by the nucleon's Landau effective mass and its first density derivative which in turn depend on the momentum-dependence of the $T=0$ single-particle energy spectrum. Recently, analytical formulas valid to next-to-leading order (NLO) effects in $T / T_{F}$

875 for all of the thermal state variables have been developed in Ref. [26], and are summarized below. 


\subsubsection{Non-relativistic models}

In non-relativistic models, the single particle spectrum has the structure

$$
\epsilon=p^{2} / 2 m_{b}+\mathcal{U}(n)+R(n, p),
$$

where $\mathcal{U}(n)$ denotes the contribution that depends only on the density $n$, whereas $R(n, p)$ contains the density- and momentum-dependent part . The implicitly temperature-dependent Landau effective mass function

$$
\mathcal{M}(p)=m_{b}\left[1+\frac{m_{b}}{p} \frac{d R(p)}{d p}\right]^{-1}
$$

is related to the Landau effective mass $m^{*}$ via

$$
\mathcal{M}\left(p=p_{F} ; T=0\right)=m^{*},
$$

where $p_{F}$ is the Fermi momentum. For a single-species system of spin $1 / 2$ particles, the leading low-temperature correction to the Fermi-liquid behavior renders the entropy density to be

$$
s \simeq 2 a n T-\frac{16}{5 \pi^{2}} a^{3} n T^{3}\left(1-L_{F}\right),
$$

where the level-density parameter $a=\pi^{2} /\left(4 T_{F}\right)=\pi^{2} m^{*} /\left(2 p_{F}^{2}\right)$, and

$$
L_{F} \equiv \frac{7}{12} p_{F}^{2} \frac{\mathcal{M}_{F}^{\prime 2}}{m^{* 2}}+\frac{7}{12} p_{F}^{2} \frac{\mathcal{M}_{F}^{\prime \prime}}{m^{*}}+\frac{3}{4} p_{F} \frac{\mathcal{M}_{F}^{\prime}}{m^{*}},
$$

where the primes denote derivatives with respect to momentum $p$. In general,

$$
\left.\frac{d \mathcal{M}(p)}{d p}\right|_{p_{F}}=\mathcal{M}_{F}^{\prime} \neq m^{* \prime}=\frac{d \mathcal{M}\left(p_{F}\right)}{d p_{F}}
$$

as $R$ can contain both $p$ and $p_{F}$ (via $n$ ). The quantity $L_{F}$ arises from nontrivial momentum dependencies in the single-particle potential. For free gases (where

$R(p)=0$ ), and for systems having only contact interactions where $R(p) \propto p^{2}$ (such as Skyrme models), $L_{F}=0$. For a multi-component system, the total entropy density is a sum of the contributions from the individual species where, in Eq. (103), the Fermi momentum, the effective mass, and its derivatives all carry a particle-species index $i$. NLO results for the entropy per particle $S$, 
thermal energy $E_{t h}$, and thermal pressure $P_{t h}$ of a single species system of spin $1 / 2$ particles are:

$$
\begin{aligned}
S & \simeq 2 a T-\frac{16}{5 \pi^{2}} a^{3} T^{3}\left(1-L_{F}\right), \\
E_{t h} & \simeq a T^{2}-\frac{12}{5 \pi^{2}} a^{3} T^{4}\left(1-L_{F}\right), \\
P_{t h} & \simeq \frac{2}{3} a n Q T^{2}-\frac{8}{5 \pi^{2}} a^{3} n Q T^{4}\left(1-L_{F}+\frac{n}{2 Q} \frac{d L_{F}}{d n}\right),
\end{aligned}
$$

where

$$
Q=1-\frac{3 n}{2 m^{*}} \frac{d m^{*}}{d n} .
$$

In simulations of astrophysical phenomena, thermal effects are often incorporated via the thermal index $\Gamma_{t h}=1+P_{t h} / \epsilon_{t h}$, where $\epsilon_{t h}$ is the thermal energy density. For non-relativistic models,

$$
\Gamma_{t h}=1+\frac{P_{t h}}{n E_{t h}} \simeq 1+\frac{2 Q}{3}-\frac{4}{5 \pi^{2}} a^{2} n T^{2} \frac{d L_{F}}{d n} .
$$

\subsubsection{Relativistic models}

The single-particle energy spectrum of relativistic mean-field theoretical (MFT) models [140] has the structure

$$
\epsilon=E^{*}+U(n), \quad E^{*}=\sqrt{p^{2}+M^{* 2}(n, T)} .
$$

The potential $U(n)$ is the result of vector meson exchanges, whereas the Dirac effective mass $M^{*}$ arises from scalar meson interactions. In this case, the NLO thermal variables are 26

$$
\begin{aligned}
S & \simeq 2 a T-\frac{16}{5 \pi^{2}} a^{3} T^{3}\left(1-L_{F}\right) \\
E_{t h} & \simeq a T^{2}-\frac{12}{5 \pi^{2}} a^{3} T^{4}\left(1-L_{F}\right), \\
P_{t h} & \simeq \frac{1}{3} a n T^{2}(1+q)-\frac{4}{5 \pi^{2}} a^{3} n T^{4}\left[1-L_{F}+q\left(1-\frac{L_{F}}{3}-\frac{10}{9} \frac{p_{F}^{4}}{E_{F}^{* 4}}\right)\right]
\end{aligned}
$$

where, using the Fermi temperature $T_{F}=p_{F}^{2} /\left(2 E_{F}^{*}\right)$,

$$
a=\frac{\pi^{2}}{4 T_{F}}, \quad q=\frac{M^{* 2}}{E_{F}^{* 2}}\left(1-\frac{3 n}{M^{*}} \frac{d M^{*}}{d n}\right) \quad \text { and } \quad L_{F}=\frac{11}{12} \frac{p_{F}^{2}}{E_{F}^{* 2}}-\frac{5}{12} \frac{p_{F}^{4}}{E_{F}^{* 4}} .
$$


Relations applicable to field theoretical calculations beyond MFT are easily derived (following the procedure adapted in the non-relativistc case) by adding to Eq. 109 contributions from two-loop contributions [26. Although such contributions do not lend themselves to analytical forms, straightforward numerical derivatives are easily calculated.

\subsection{Illustrative Results}

In the results above, the entropy density and specific heats are carried to $\mathcal{O}\left(T / T_{F}\right)^{3}$ whereas the energy density and pressure to $\mathcal{O}\left(T / T_{F}\right)^{4}$, extending the leading order results of FLT. These extensions employed a generalized Landau effective mass function which enables the calculation of the entropy density, and thereafter the other state variables from Maxwell's relations, for a general single-particle spectrum. For models with contact interactions, knowledge of the Landau effective mass suffices, whereas for models with finite-range interactions, momentum derivatives of the Landau effective mass function up to second or third order are required.

The value of the NLO results lies in the fact that thermal effects in the near-degenerate to degenerate limits can be calculated from the $T=0$ singleparticle spectra from microscopic calculations (e.g., non-relativistic and relativistic Brueckner-Hartree-Fock calculations or effective field theoretical models) which may prove time consuming at finite $T$. To illustrate this advantage, models that are widely used in nuclear and neutron star phenomenology were chosen in Ref. 26] for demonstration purposes.

\subsubsection{Non-relativistic models}

In the category of non-relativistic potential models, the finite-range model, referred to as $\mathrm{MDI}(\mathrm{A})$ [16, 141, that reproduces the empirical properties of isospin symmetric and asymmetric bulk nuclear matter [23, optical model fits to nucleon-nucleus scattering data 142, heavy-ion flow data in the energy range 0.5-2 GeV/A [22], and the largest well-measured neutron star mass of $2 \mathrm{M}_{\odot}$, was contrasted in Ref. [26] with a conventional zero-range Skyrme model known 

models. For both MDI(A) and $\mathrm{SkO}^{\prime}$ models, the effect of a finite $x$ [Fig. 2(b)] is more pronounced at low momenta for which the single particle potential becomes more attractive relative to that for pure neutron matter.

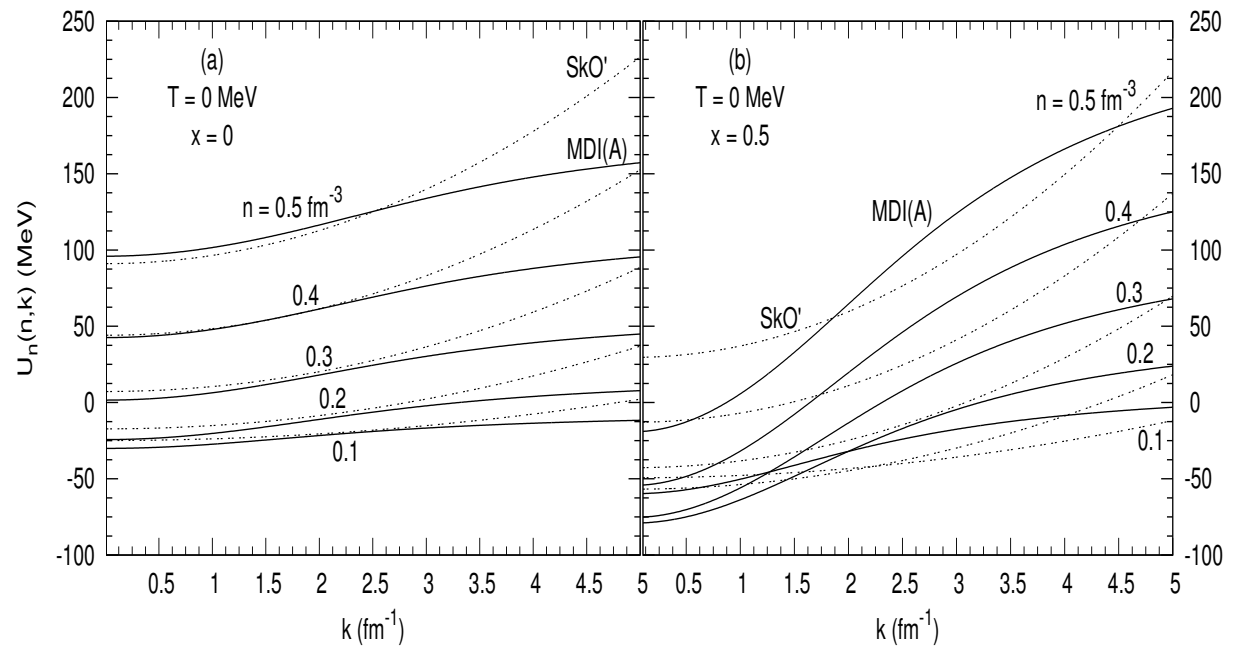

Figure 13: Neutron single particle potentials vs momentum at $T=0$ for the densities $n$ and proton fractions $x=n_{p} / n$ as marked. Figure from Ref. 23].

The neutron Landau effective masses $m_{n}^{*}$ (scaled with its vacuum value) and their logarithmic derivatives with respect to density are shown as functions of $n$ in Fig. 14. Noteworthy points here are: (i) The isospin splittings are 
qualitatively similar $-m_{n}^{*} / m_{b}$ being larger for PNM than for SNM - although quantitative differences are present, and (ii) except for $n$ up to $0.2 \mathrm{fm}^{-3}$, the decrease with increasing $n$ for the MDI model is relatively slow (logarithmic decline) compared with that for the $\mathrm{SkO}^{\prime}$ model $\left[(1+\text { constant } \times \mathrm{n})^{-1}\right.$ fall off] . The logarithmic derivatives $m_{n}^{*}$ for MDI(A) show little variation with $n$ at supranuclear densities, whereas results for the $\mathrm{SkO}^{\prime}$ model, which take the simple form $\left(m_{n}^{*} / m_{b}\right)-1$, show a significant variation with $n$. The density dependences of the effective masses and their logarithmic derivatives determine the behavior of all the thermal properties in FLT. Higher order derivatives of the Landau effective mass function in Eq. 101) appear in FLT+NLO.

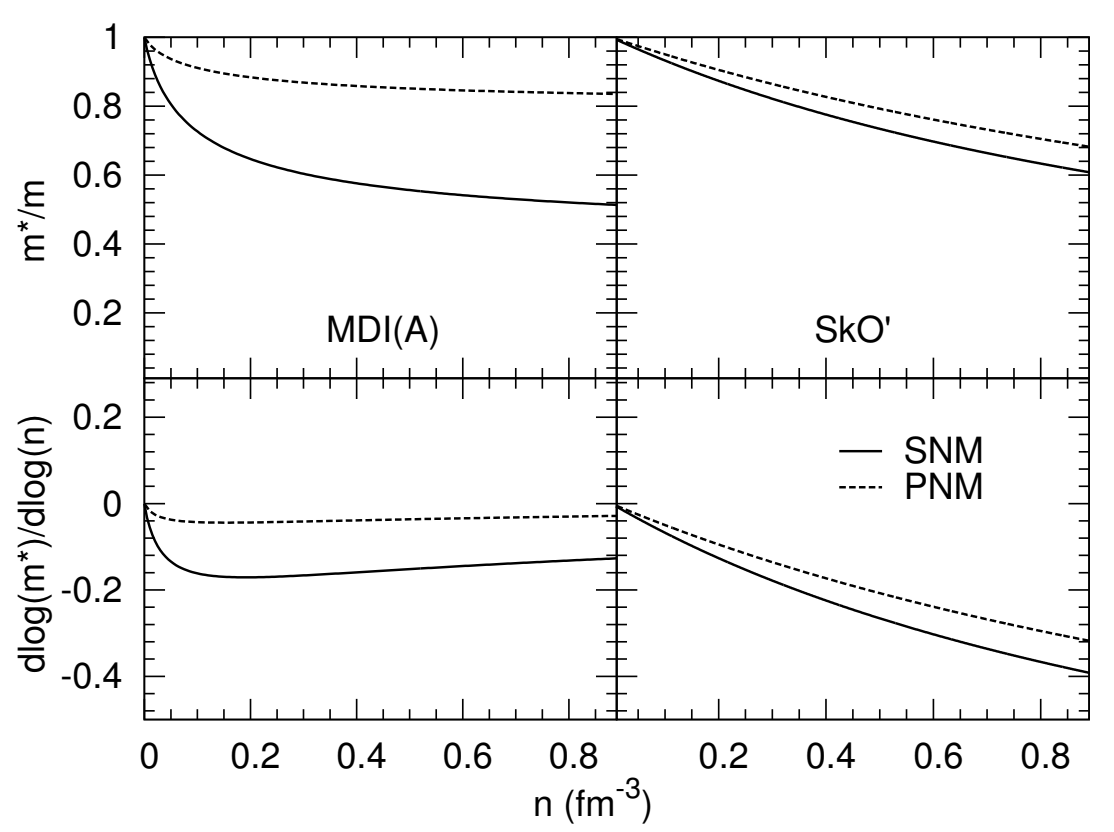

Figure 14: The neutron effective masses (top panels) and their logarithmic derivatives (bottom panels) for non-relativistic potential models ( $\mathrm{MDI}(\mathrm{A})$ and $\mathrm{SkO}^{\prime}$ ) for symmetric nuclear matter (SNM) and pure neutron matter (PNM). Figure extracted from Ref. [26].

The FLT and FLT+NLO results for the thermal energy, $E_{t h}$, and the thermal pressure, $P_{t h}$ are compared with the exact numerical results in Figs. 15 and 16 . The NLO corrections yield agreement with the exact results down to densities of $(0.5-1) n_{s}$ compared to $(2-3) n_{s}$ for FLT. As with FLT, systematically better 
agreement with FLT+NLO occurs for PNM than for SNM owing to the neutron density in PNM $\left(n_{n}=n\right)$ being twice that in SNM $\left(n_{n}=n / 2\right)$; PNM is more degenerate than SNM at the same $n$. The efficacy of FLT+NLO is such that it reproduces the exact results for all $(n, T)$ for which the entropy per particle $S \leq 2$, whereas FLT does so only for $S \leq 1$.

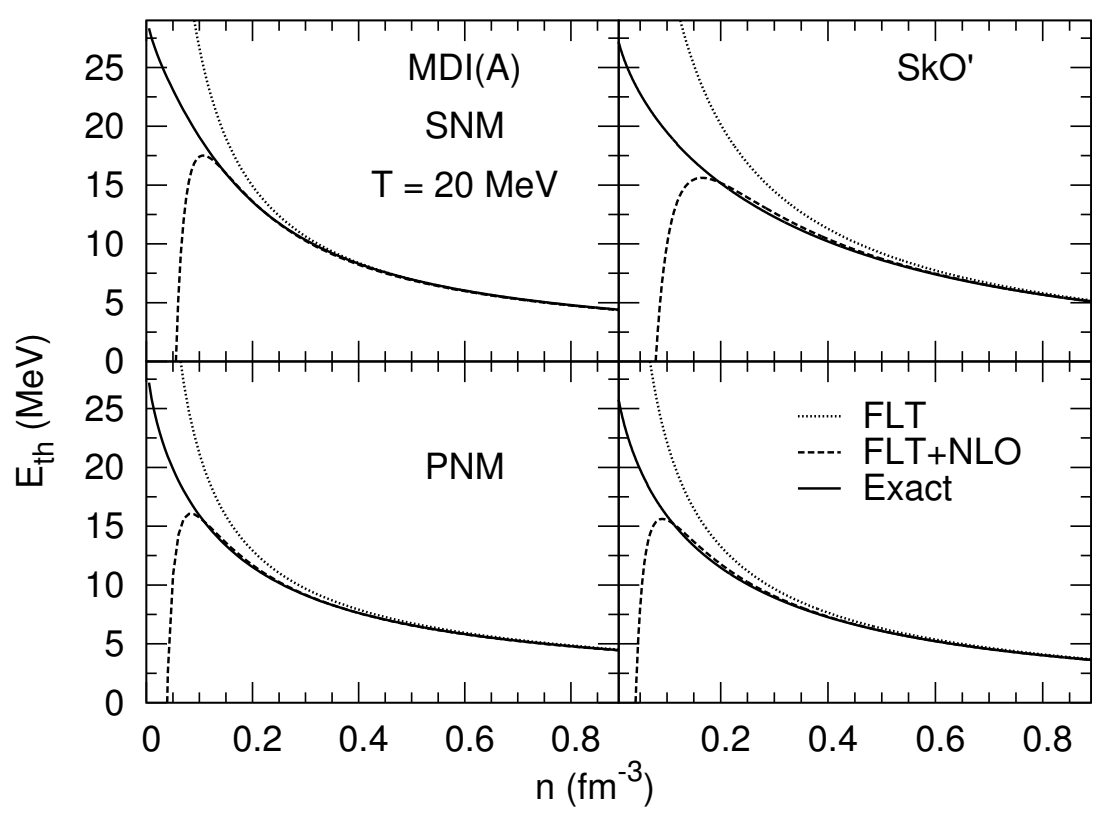

Figure 15: Thermal energy for the non-relativistic models (MDI(A) and $\mathrm{SkO}^{\prime}$ ) at a temperature of $T=20 \mathrm{MeV}$. Results for SNM are in the top panels and for PNM in the bottom panels. Figure extracted from Ref. [26].

Currently, many astrophysical simulations incorporate thermal effects through the use of the thermal index $\Gamma_{t h}=1+P_{t h} / \epsilon_{t h}$ [144, 145, 146, 147, 148]. In Fig. 17, results of $\Gamma_{t h}$ vs $n$ in matter with only nucleons $(n p)$, and with leptons and photons included (nper) are contrasted for representative values of $T$ and the proton fraction $x=Y_{e}$. The results shown are from exact numerical calculations for the non-relativistic models $\mathrm{MDI}(\mathrm{A})$ and $\mathrm{SkO}^{\prime}$, which agree with the non-degenerate and degenerate limit expressions discussed above very well in their respective regions of applicability (see Refs. [23, 26] for quantitative analyses). For $n<10^{-2} \mathrm{fm}^{-3}$, effects of interactions in $n p$ matter are small 


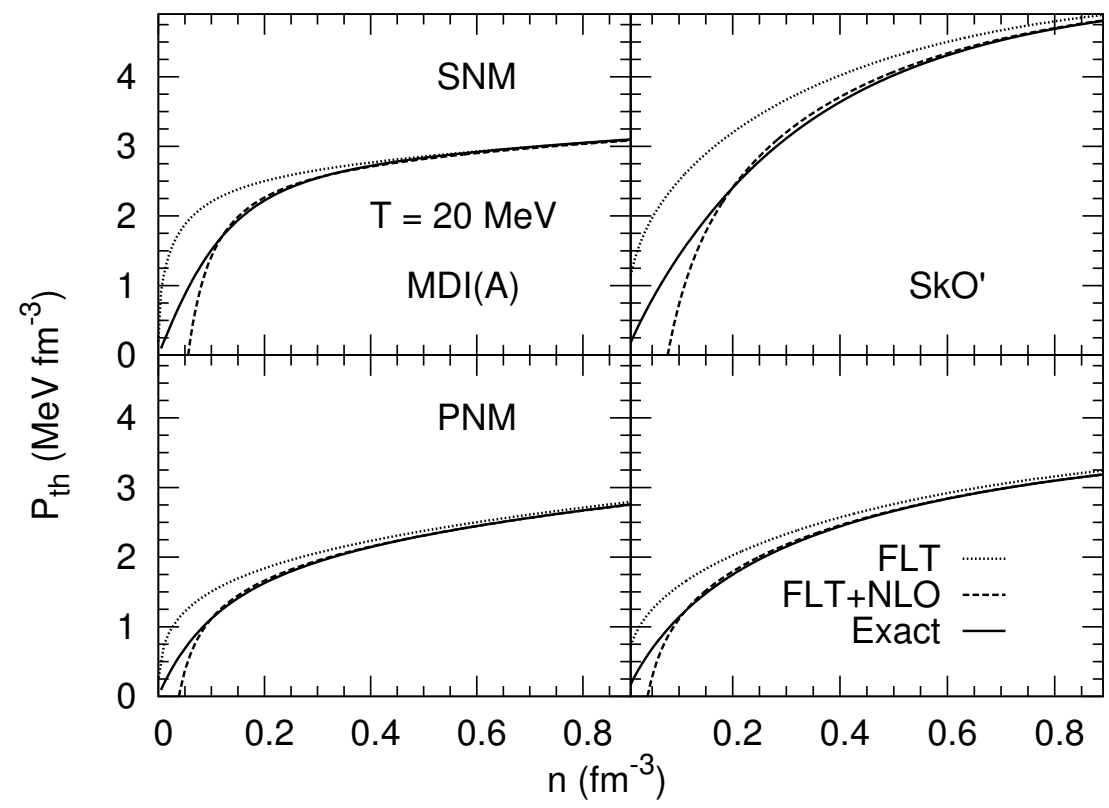

Figure 16: Same as Fig. 15 but for thermal pressure. Figure extracted from Ref. 26].

and $\Gamma_{t h} \rightarrow 5 / 3$, the value for non-relativistic ideal fermions. The presence of relativistic electrons and photons, for which $\Gamma_{t h}=4 / 3$, causes the total $\Gamma_{t h}$ to tend toward 4/3 in npe $\gamma$ matter as $n$ decreases progressively from $10^{-2} \mathrm{fm}^{-3}$. In the approximate range $10^{-2} \mathrm{fm}^{-3}<n<10^{-1} \mathrm{fm}^{-3}$ and for $T<15 \mathrm{MeV}$, an inhomogeneous phase consisting of nuclei, pasta-like configurations, and nper (shown by the shaded region for $T=10 \mathrm{MeV}$, but not considered in the calculations) is energetically preferred over a uniform phase of npe $\gamma$. Trace amounts of $\alpha$-particles may be present even at $T=20 \mathrm{MeV}$ in this density region. For $T \geq 15 \mathrm{MeV}$, inhomogeneous phases give way to a uniform phase pf $n p e \gamma$ for all densities.

The differences in $\Gamma_{t h}$ for near-nuclear to supra-nuclear densities in $n p$ matter for the $\mathrm{MDI}(\mathrm{A})$ and $\mathrm{SkO}^{\prime}$ models are caused by the differences in the behaviors of their effective masses (see Fig. 14) which control the thermal properties. The mild variation of $\Gamma_{t h}$ for the $\operatorname{MDI}(\mathrm{A})$ model is because of the relatively flat behavior of $m^{*}$ and $d \ln m^{*} / d \ln n$ with increasing $n$. In contrast, the rapid rise 


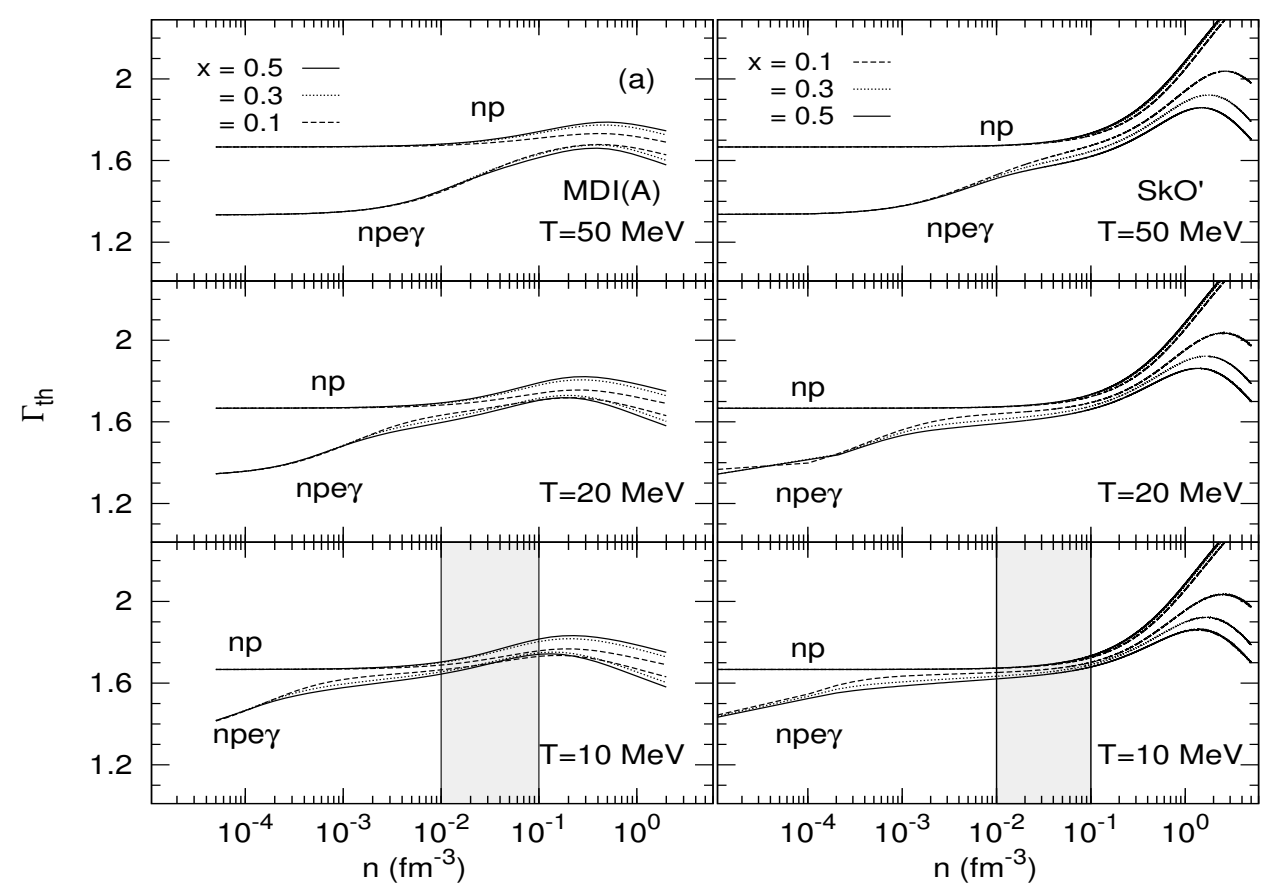

Figure 17: The thermal index $\Gamma_{t h}$ for matter with only nucleons (np), and with leptons and photons (npe $\gamma$ ) for the non-relativistic models $\mathrm{MDI}(\mathrm{A})$ and $\mathrm{SkO}^{\prime}$. For $T=10 \mathrm{MeV}$, the shaded region shows the range of densities in which nuclei and pasta-like configurations (not considered here) exist. Figure extracted from Ref. 23].

of $\Gamma_{t h}$ for the $\mathrm{SkO}^{\prime}$ model is a consequence of the monotonic decrease with $n$ of both $m^{*}$ and $d \ln m^{*} / d \ln n$. Results of both of these models are, however, modified in nper matter with a tendency to approach the value $4 / 3$ for very large densities after reaching a peak value.

\subsubsection{Relativistic field-theoretical models}

Contrasting the above results with those of relativistic field-theoretical models is taken up below. Results of mean-field theoretical (MFT) calculations from Refs. 23, 26] are summarized first, and thereafter the recent results of two-loop (TL) calculations performed in Ref. [149]. The TL calculations incorporate exchange-diagram contributions arising from scalar $(\sigma)$, vector $(\omega)$, iso-vector $(\rho)$ and $\pi$-meson interactions between nucleons. In the non-relativistic limit, these contributions are formally the same as in non-relativistic treatments with 
finite-range interactions.

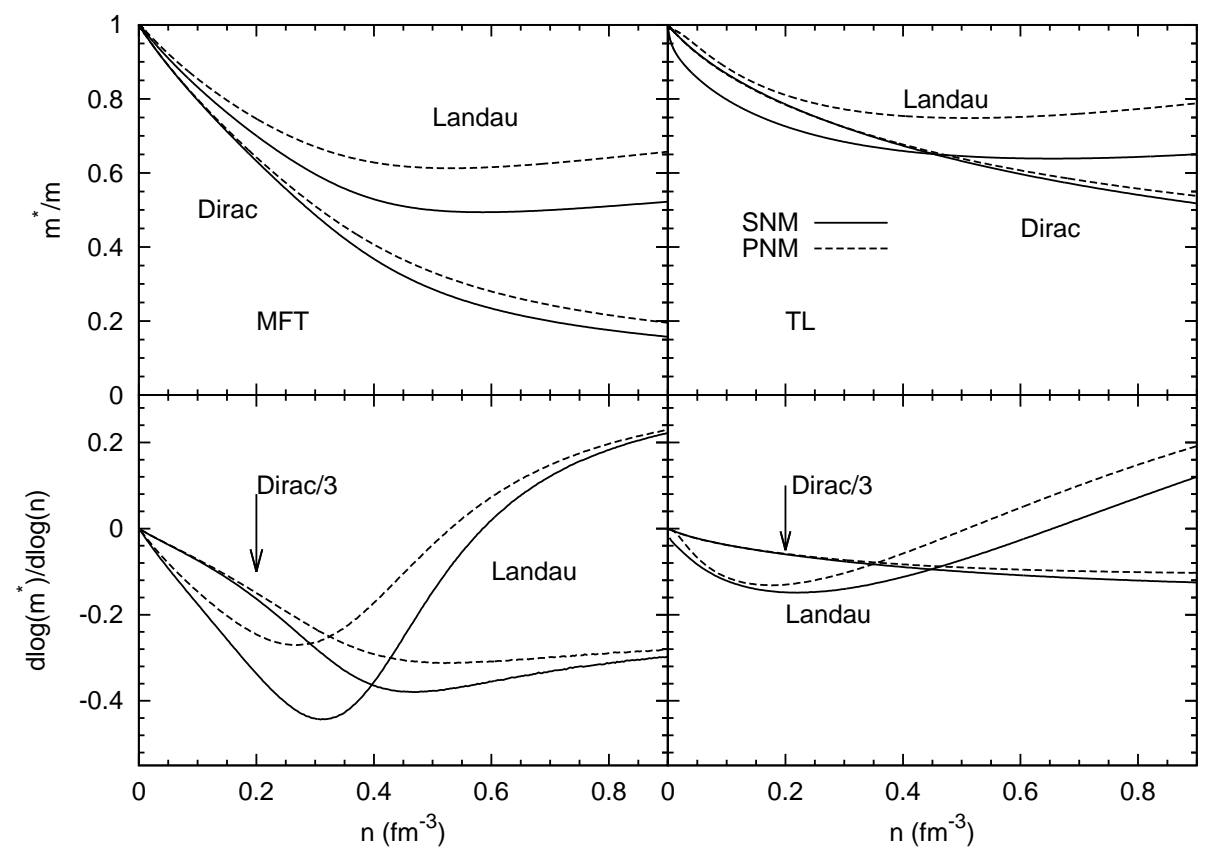

Figure 18: The neutron effective masses (top panels) and their logarithmic derivatives (bottom panels) for field-theoretical models at the MFT and TL levels for symmetric nuclear matter (SNM) and pure neutron matter (PNM). Figure courtsey Xilin Zhang.

In Fig. 18 are shown the Dirac effective masses $M^{*}$, the Landau effective masses $m^{*}$, and their logarithmic derivatives as functions of $n$ for SNM (solid curves) and PNM (dashed curves) [149] in MFT. At $T=0$ in MFT,

$$
m^{*}=E_{F}^{*}=\sqrt{p_{F}^{2}+M^{* 2}} \quad \text { and } \quad \frac{d \ln m^{*}}{d \ln n}=\frac{1}{3}\left[1-\frac{M^{* 2}}{E_{F}^{* 2}}\left(1-3 \frac{d \ln M^{*}}{d \ln n}\right)\right] .
$$

The density-dependent $M^{*}$ is obtained by minimizing the total energy density (pressure at $T \neq 0$ ) with respect to $M^{*}$. The asymptotic behaviors for $n / n_{0} \gg 1$ are: $m^{*} \rightarrow p_{F}\left(\right.$ as $\left.M^{*} \rightarrow 0\right)$ and $d \ln m^{*} / d \ln n \rightarrow 1 / 3$. The approach to these limits is evident from the results in Fig. 18 . The density $n_{\min }$ at which $m^{*}$ is a minimum is obtained from

$$
\frac{p_{F}}{M^{*}}+\frac{d M^{*}}{d p_{F}}=0,
$$

which for the MFT model considered occurs at $n=0.59(0.53) \mathrm{fm}^{-3}$ for SNM 
(PNM). The density $n_{R}$ for which $p_{F}=M^{*}$ (whence $m^{*}=\sqrt{2} p_{F}$ ) occurs at

$$
n_{R}=\frac{\gamma}{6 \pi^{2}}\left(\frac{m_{b}}{\sqrt{2} \hbar}\right)^{3}\left(\frac{m^{*}}{m_{b}}\right)^{3} \mathrm{fm}^{-3}=0.643 \gamma\left(\frac{m^{*}}{m_{b}}\right)^{3} \mathrm{fm}^{-3},
$$

where $\gamma=4(2)$ for SNM (PNM), and which is about $(2 / 3) n_{\min }=0.39(35) \mathrm{fm}^{-3}$ for SNM (PNM) and marks the transition of nucleons well into the relativistic region.

The inclusion of two-loop effects significantly changes the behavior of $M^{*}$, $m^{*}$, and their logarithmic derivatives, their variation with $n$ being slower than in MFT (see the right hand panels in Fig. 18). Both $M^{*}$ and $m^{*}$ acquire substantially larger values than those in MFT for all $n$ in both SNM and PNM. The densities at which $m^{*}$ 's attain their minima are similar to those in MFT, but the densities $n_{R}$ at which $M^{*}=p_{F}$ shift to considerably larger densities than in MFT. Consequently, the thermal properties are correspondingly influenced. An analytical analysis of $m^{*}$ 's similar to that for MFT given above is precluded because of the inherently complicated structure of the TL integrals which require numerical calculations. Their overall effects are, however, similar to those in which exchange contributions from finite-range interactions are included in non-relativistic treatments such as the $\operatorname{MDI}(\mathrm{A})$ model discussed before. This similarity is not unexpected as the TL contribution to the total energy density (minimizing which $M^{*}$ is obtained) reduces formally to its structure in non-relativistic treatments that include exchange interactions [149].

The thermal index $\Gamma_{t h}$ vs $n$ for MFT and TL models are shown in Fig. 19 Results with and without the inclusion of leptons and photons in this figure are for the same proton fractions $x=Y_{e}$ and $T$ as in Fig. 17 for non-relativistic models. The analysis of $\Gamma_{t h}$ vs $n$ for $n p$ matter for the MFT model is facilitated by its degenerate limit expression $[23,26]$

$$
\Gamma_{t h}=\frac{4}{3}+\frac{1}{3}\left[\left(\frac{M^{*}}{E_{F}^{*}}\right)^{2}\left(1-3 \frac{d \ln M^{*}}{d \ln n}\right)\right]+\text { NLO corrections . }
$$

In the non-relativisitic limit, $M^{*} / E_{F}^{*} \rightarrow 1$ and the logarithmic derivative of $d \ln M^{*} / d \ln n \rightarrow 0$ leading to $\Gamma_{t h}=5 / 3$. In the ultra-relativistic limit, $M^{*} \rightarrow 0$ 


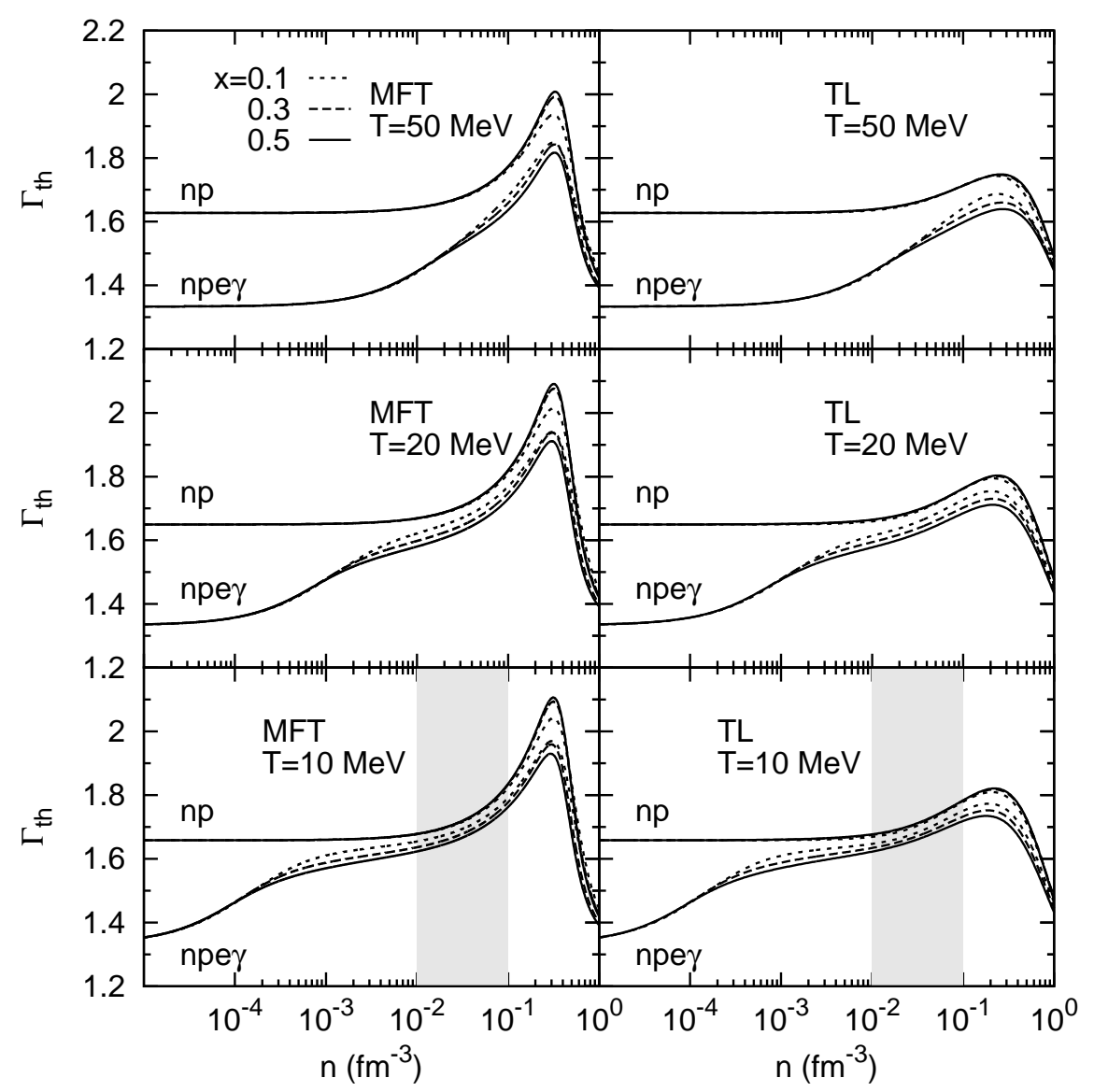

Figure 19: The thermal index $\Gamma_{t h}$ from relativistic MFT and TL models. Results show contrasts between matter with only nucleons (np), and with leptons and photons (npe $\gamma$ ). Figure courtesy Xilin Zhang.

so that $\Gamma_{t h}=4 / 3$. The density at which the maximum occurs in $\Gamma_{t h}$ (for SNM or PNM) can be determined from

$$
\frac{d \Gamma_{t h}}{d p_{F}}=0=\frac{d}{d p_{F}}\left(\frac{p_{F}^{2}}{E_{F}^{* 2}}+\frac{p_{F} M^{*}}{E_{F}^{* 2}} \frac{d M^{*}}{d p_{F}}\right) .
$$

The result is $n \simeq 0.3 \mathrm{fm}^{-3}$, which also holds for all $x$ in good agreement with the results of exact numerical calculations. The sub-leading NLO corrections do not affect these numbers [26]. With increasing values of $T$ (e.g., $50 \mathrm{MeV}$ in the figure), $M^{*}$ begins to acquire a $T$-dependence, so the values of $\Gamma_{t h}$ differ slightly from that given by Eq. 115, particularly below the peaks. 
Leptons and photons are relativistic components in nper matter each with $\Gamma_{t h}=4 / 3$. Except at very high $T$ 's, the contribution of photons is small, but that of leptons is significant in charge neutral matter for all $n$. Their presence in a mixture reduces $\Gamma_{t h}$ toward $4 / 3$ at asymptotically low and high densities as is evident from the results in Fig. 19 (and Fig. 17). The peak values of $\Gamma_{t h}$ are also reduced from their values in $n p$ matter as in the non-relativistic case. The MFT results for $\Gamma_{t h}$ differ substantially from the non-relativistic cases presented before at near-nuclear and supra-nuclear densities.

The influence of TL effects on $\Gamma_{t h}$ is shown in the right hand panels of Fig. 1025 19. From near-nuclear to supra-nuclear densities and for all T's shown, TL effects reduce $\Gamma_{t h}$ from its values in MFT in $n p$ matter. This reduction is a consequence of the much larger values of $M^{*}$ 's and $m^{*}$ 's (which determine the thermal properties) in TL calculations compared to those in MFT. The presence of leptons and photons leads to a further reduction of $\Gamma_{t h}$ at all $n$ and for all

${ }_{1030} x$. These results are semi-quantitatively similar to those of the non-relativistic MDI(A) model (see Fig. 17) in which exchange contributions from finite-range interactions were considered.

\subsection{General Comments}

The conclusions that emerge from the results of both non-relativistic and relativistic field-theoretical models are: (i) the presence of electrons and photons results in an overall reduction of $\Gamma_{t h}$ in $n p$ matter for all $n$, (ii) variations of $\Gamma_{t h}$ with $x=Y_{e}$ and $T$ are relatively small, and (iii) variations with $n$ are significant, but depend sensitively on the density dependence of the Landau effective mass $m^{*}$ of the underlying model; the more rapidly $m^{*}$ decreases with $n$, the larger 1040 is the variation in $\Gamma_{t h}$.

It was mentioned in the introduction the interest Gerry had in effective masses. A series of papers published in the 1980's addressed this issue and brought to light the separate roles of the momentum- and energy-dependences of effective masses in nuclei and nuclear matter [150, 151, 152, 153, 154]. Relatively few calculations of thermal effects performed so far for astrophysical 
applications have considered effects from long-wavelength fluctuations, single particle-hole excitations, or collective and pairing correlations near the Fermi surface [155, 139. These many-body correlations generally lead to an enhancement of the effective mass at the Fermi surface and therefore influence the entropy, and, hence, the other state variables. These correlations are not captured in mean-field theories even when exchange contributions are included in either non-relativistic or relativistic models. Pethick and Carneiro [155] have shown that long-wavelength fluctuations lead to non-analytic behavior of the quasiparticle interaction which in turn gives rise to $T^{3} \ln T$ terms in the specific heat and entropy. Such terms then become the leading correction to the results of Fermi-liquid theory at low $T$. In liquid ${ }^{3} \mathrm{He}$, use of only $T$ and $T^{3} \ln T$ terms gives a remarkably good fit to specific heat data (see Fig. 1.8 in Ref. [139]).

Fantoni et al. [156] used correlated basis functions (CBF) to study the nucleon optical potential in nuclear matter and found that the enhancement of the effective mass appears to be much smaller than in liquid ${ }^{3} \mathrm{He}$. A comparison of single-particle energies for variational calculations with and without secondorder CBF does not show significant differences (Ref. [156], Figure 6). Since enhancements of the effective mass and the $T^{3} \ln T$ term have the same origin, it seems likely that contributions from the $T^{3} \ln T$ term are not large in dense nuclear matter. Green's function Monte Carlo calculations of finite-temperature matter are not yet available owing to the fermion-sign problem. Nevertheless, this topic deserves further scrutiny in light of modern developments in effective field theories of nuclear matter, at least up to the densities for which they are reliable. Contributions from these sources should be added to those reported in this article when deemed appropriate.

\section{NON-THERMAL EFFECTS IN BULK MATTER}

Bulk nucleonic matter is also encountered in medium-energy heavy-ion collisions at beam energy per particle in the range $0.5-2 \mathrm{GeV}$. In these collisions, densities up to 3-4 times the nuclear equilibrium density are accessed albeit 
in non-equilibrium conditions. The matter, momentum, and energy flow of nucleons in such collisions has been characterized by (i) the mean transverse momentum per nucleon $\left\langle p_{x}\right\rangle / A$ vs rapidity $y / y_{\text {proj }}$ [157, (ii) flow angle from asphericity analysis [158, (iii) azimuthal distributions [16], and (iv) radial flow [159. In recent years, the analysis of data has been extended to include the collective flow of momentum in terms of Fourier coefficients (proposed in Ref. [160]) similar to those extracted for relativistic heavy-ion collision experiments at BNL and LHC, but at much higher energies.

Attempts to account for the experimental findings in medium-energy heavyion collisions either through cascade calculations (sequential scatterings of nucleons with their free-space cross-sections) or through hydrodynamical calculations [161] (which presume local thermal equilibrium with the EOS as an input) failed insofar as they predicted too little or too much collective flow. The more fundamental kinetic description introduced in Ref. [14] employed Monte-Carlo methods to solve for the phase space distribution function $f(\vec{r}, \vec{p}, t)$ of a nucleon via the Boltzmann-Uhling-Uhlenbeck (BUU) equation incorporating both the mean-field $U$ and a collision term with Pauli blocking of final states:

$$
\begin{aligned}
\frac{\partial f}{\partial t} & +\vec{\nabla}_{p} U \cdot \vec{\nabla}_{r} f-\vec{\nabla}_{r} U \cdot \vec{\nabla}_{p} f= \\
& -\frac{1}{(2 \pi)^{3}} \int d^{3} p_{2} d^{3} p_{2^{\prime}} d \Omega \frac{d \sigma_{N N}}{d \Omega} v_{12} \delta^{3}\left(\vec{p}+\vec{p}_{2}-\vec{p}_{1^{\prime}}-\vec{p}_{2^{\prime}}\right) \\
& \times\left[f f_{2}\left(1-f_{1^{\prime}}\right)\left(1-f_{2^{\prime}}\right)-f_{1^{\prime}} f_{2^{\prime}}(1-f)\left(1-f_{2}\right)\right] .
\end{aligned}
$$

The mean field $U$, the functional derivative of the energy density $\mathcal{H}$ of matter at zero temperature, i.e., $U(n, p) \equiv \delta \mathcal{H} / \delta n$, depends on both the local density $n$ and the nucleon momentum $\vec{p}$, and serves as an input. The other physical input is the nucleon-nucleon differential cross section, $d \sigma_{N N} / d \Omega$, which depends on the relative velocity $v_{12}$. The evolution of $f(\vec{r}, \vec{p}, t)$ is inherently off-equilibrium.

Fig. 20 shows an example of comparison between data and theoretical calculations in which isospin averaged cross sections and a momentum-dependent mean field of symmetric nuclear matter were employed [17]. It is worth noting that in these experiments, particle detection inefficiencies caused the experi- 

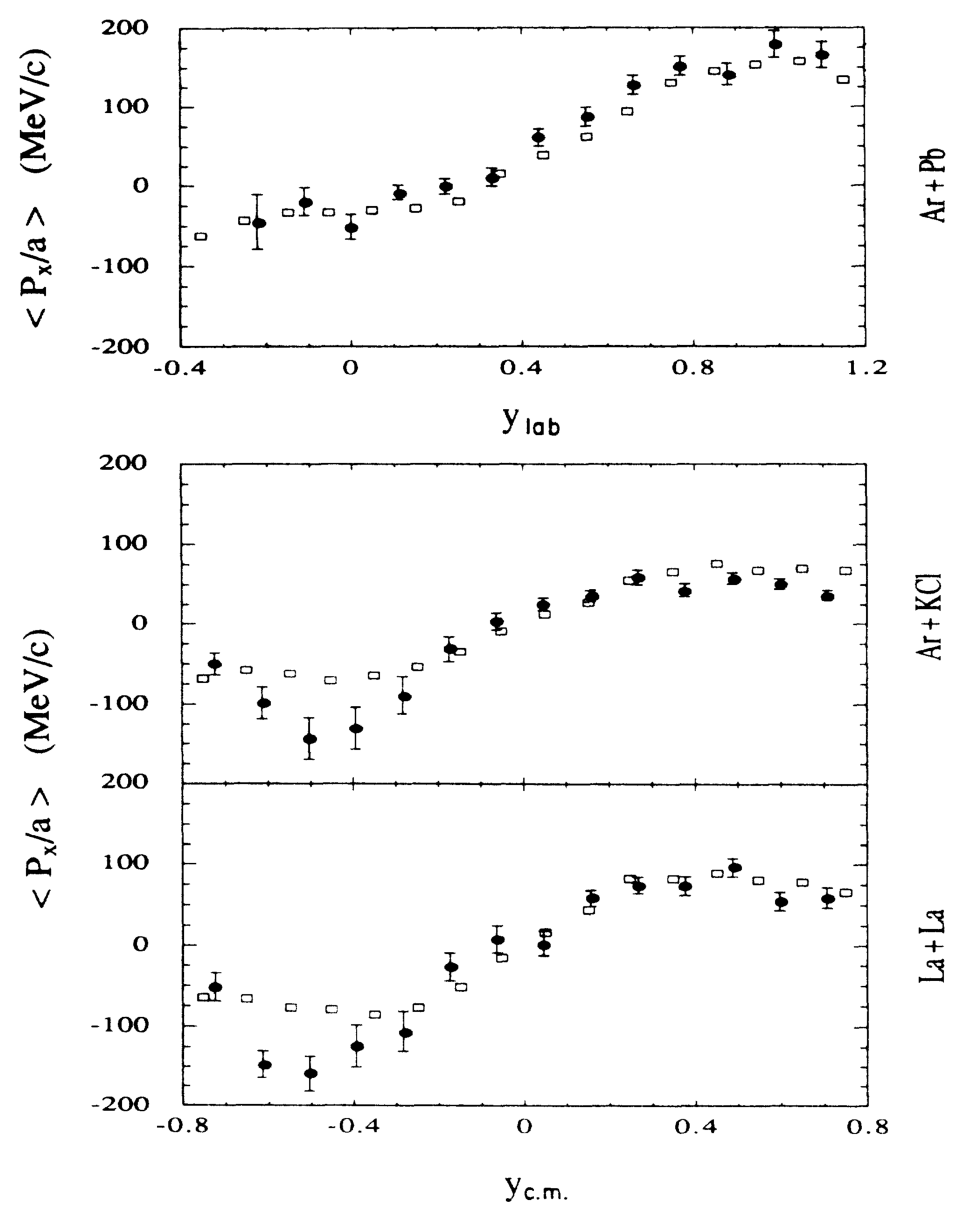

Figure 20: Transverse momentum per nucleon as a function of rapidity in reactions of 800 $\mathrm{MeV}$ per projectile nucleon. Results of BUU simulations with the MDYI interaction (open squares) are compared with the data of Ref. [19] (solid circles). Results for $\mathrm{Ar}+\mathrm{Pb}$ are in the lab, for $\mathrm{La}+\mathrm{La}$ and $\mathrm{Ar}+\mathrm{KCl}$ in the center of mass. Figure from Ref. [17.

mental transverse momenta in the backward direction to be artificially biased towards large negative values and were therefore unreliable. The interplay of the so-called Vlasov term [second term in Eq. (117) featuring $U(n, p)]$ and collisions [the right hand side in Eq. [117]] was found to be crucial to account for the data. Most of the sideways flow gauged through $\left\langle p_{x}\right\rangle / A$ vs $y / y_{\text {proj }}$ is built during the early stages of the collision under non-equilibrium conditions.

Studies performed in Refs. [13, 15, 16, 17, 20, 21] have shed valuable insight into the nature of the momentum-dependence in the mean field $U(n, p)$ 
required to account for the collective flow observed in heavy-ion data. For example, a quadratic momentum-dependence in $U(n, p)$, characteristic of zero-range Skyrme models, gives too much sideways flow [15]. Relativistic MFT models for which the Schrodinger-equivalent potential is linear in energy [162, 11] also give too much sideways flow. A mean field that saturates at high momenta, such as those in finite-range models, adequately accounts for the data. A similar mean field is also required in optical model fits of nucleon-nucleus scattering data [142, 163, 20, 21]. Examples of microscopic calculations of $U(n, p)$ that show a saturating behavior at high momenta in non-relativistic approaches can be found in Refs. [18, 164. A similar behavior is observed both in MFT models that employ non-linear derivatives [165] and in the two-loop calculations of Ref. [149].

The conclusion that has emerged is that as long $U(n, p)$ saturates at high momenta as required in optical model fits to nuclear data, a symmetric matter incompressibility parameter $K_{s} \approx 240 \mathrm{MeV}$ fits the heavy-ion data [22]. This is comforting in view of the fact that the value $K_{s} \simeq 240 \pm 20 \mathrm{MeV}$ is suggested by the analysis of the giant monopole resonance data [5, 6, 7]. Additionally, it has been confirmed that an EOS based on such a mean field can yield a neutron star with $M_{\max } \gtrsim 2 M_{\odot}[23$.

Rare-isotope accelerators (RIAs) that can collide highly neutron-rich nuclei has encouraged work to study collisions featuring high neutron excess [141, 166, 167. Generalizing Eq. 117) to a mixture, the kinetic equation for neutrons is

$$
\frac{\partial f_{n}}{\partial t}+\vec{\nabla}_{p} U \cdot \vec{\nabla}_{r} f_{n}-\vec{\nabla}_{r} U \cdot \vec{\nabla}_{p} f_{n}=J_{n}=\sum_{i=n, p} J_{n i},
$$

where $J_{n}$ describes collisions of a neutron with all other neutrons and protons. A similar equation applies for protons with appropriate modifications. The mean field $U \equiv U\left(n_{n}, n_{p} ; \vec{p}\right)$ now depends explicitly on the neutron-proton asymmetry and establishes a connection to the symmetry energy in that $U$ is now obtained from a functional differentiation of the Hamiltonian density $\mathcal{H}\left(n_{n}, n_{p}\right)$ of isospin asymmetric matter. Examples of such mean fields may be found in Refs. 168, 166, 167]. Isospin asymmetry influences the neutron-proton 


\section{CONCLUSIONS}

The advances in dense matter theory during the time that Gerry Brown was interested in the topic were enormous, and the pace of advancement has not slowed. Astronomical observations have provided the important evidence that the neutron-rich EOS above $n_{s}$ is quite stiff, being able to support neutron star masses in excess of $2 M_{\odot}$. At the same time, the EOS near $n_{s}$ seems to be relatively soft, judging from experimental evidence and neutron matter studies indicating $40 \mathrm{MeV} \lesssim L \lesssim 70 \mathrm{MeV}$. Assuming that neutron stars have crusts, neutron matter calculations are reasonable correct near $n_{s}$, causality is valid, and GR is the correct theory of gravity at the required densities, typical neutron star radii are confined to the range $9 \mathrm{~km}<R_{1.4}<14 \mathrm{~km}$. If strong phase transitions do not exist in the range $n_{s}<n<2 n_{s}$, this range is even smaller: $10.7 \mathrm{~km} \lesssim R_{1.4} \lesssim 13.1 \mathrm{~km}$. Many upcoming astrophysical observations should be able to confirm these predictions and reduce these ranges further, allowing for a tighter description of neutron star matter and its composition.

Further evidence concerning the internal composition of neutron stars is becoming available from observations of neutron star cooling, which consists of the body of observed surface temperatures and ages of a few dozen neutron stars to date. In one spectacular case, the neutron star in the supernova remnant

1160 Cassiopeia A has, over the period from 2000-2010, been observed [169] to be cooling much faster than expected for a star it's age ( $\sim 330$ years). While it has been suggested from examining observations from a variety of detectors that this cooling represents an anomalous instrumental effect [170, continued obser- 
vations for an additional five years [171], employing one carefully calibrated detector (Chandra's ACIS-S), have reinforced the original report of rapid cooling. This rapid cooling has been interpreted [172, 173] to be caused by a temporary phase of enhanced neutrino emission due to Cooper pair breaking and formation during the onset of neutron superfluidity in the star's core. Furthermore, the rapidity of the cooling suggests that not only did it begin within a few decades of the present time, but that protons must be superconducting in the interior as well. It is possible to determine the critical temperature for the neutron superfluid with some accuracy $\left(T_{c}=5-10 \times 10^{8} \mathrm{~K}\right)$ since it depends mostly on the well-determined age of this neutron star ${ }^{28}$ Continued observations of this star, and other cooling neutron stars, will reveal further information about their interiors and the thermal properties of dense matter.

The physics and astrophysics of core-collapse supernovae, the birth of neutron stars and their evolution to old age, and mergers of binary stars involving neutron stars and black holes, also involve thermal effects to varying degrees. In addition to influencing the hydrodynamic evolutions of these objects, thermal effects play significant roles in the photon, neutrino, and gravitational radiation emissivities at different stages of their evolutions. With vastly improved capabilities in astronomical observations and laboratory experiments involving rare-isotope accelerators that can access highly neutron-rich matter, we are now well poised to explore and establish the properties of dense hadronic matter under conditions of extreme density, isospin content, temperature, and magnetic fields.

\section{ACKNOWLEDGEMENTS}

This work was supported by the U.S. DOE under Grants No. DE-FG0287ER-40317 and DE-FG02-93ER-40756. The authors are grateful for the generous help of collaborators Constantinos Constantinou, Yeunhwan Lim, Brian

\footnotetext{
${ }^{28}$ The more recent analysis of Ref. [171] suggests $T_{c} \simeq 6 \times 10^{8} \mathrm{~K}$.
} 
Muccioli, Andrew Steiner, and Xilin Zhang. We are also grateful to collaborators Christian Drischler and Achim Schwenk for providing us with prepublication results from theoretical neutron and nuclear matter studies of arbitrary proton fractions, and to Xilin Zhang for prepublication results concerning thermal properties of matter.

\section{REFERENCES}

[1] G. E. Brown, E. Osnes, Phys. Lett. 159B (1985) 223.

[2] J. P. Blaizot, Phys. Rep. 64 (1980) 171.

[3] P. Demorest, T. Pennucci, S. Ransom, M. Roberts, J. Hessels, Nature 467 (7319) (2010) 1081-1083.

[4] J. Antoniadis, P. C. C. Freire, N. Wex, et al., Science 340 (6131).

[5] D. H. Youngblood, H. L. Clark, Y.-W. Lui, Phys. Rev. Lett. 82 (1999) 691.

[6] U. Garg, Nucl. Phys. A731 (2004) 3-14.

1205

[7] G. Colo, N. Van Giai, J. Meyer, K. Bennaceur, P. Bonche, Phys. Rev. C 70 (2004) 024307.

[8] H. A. Bethe, G. E. Brown, J. A. Applegate, J. M. Lattimer, Nucl. Phys. A $324(1979) 487$.

[9] J. M. Lattimer, Nucl. Phys. A 938 (2014) 276.

1210 [10] K. Langanke, G. Marinez-Pínedo, Nucl. Phys. A 938 (2014) 305.

[11] T. L. Ainsworth, E. Baron, G. E. Brown, J. Cooperstein, M. Prakash, Nucl. Phys. A464 (1987) 740.

[12] J. Aichelin, A. Rosenhauer, G. Pielert, H. Stöcker, G. W., Phys. Rev. Lett. 58 (1987) 1926. 
1215

[14] G. F. Bertsch, S. Das Gupta, Phys. Rep. 160 (1988) 189.

[15] M. Prakash, T. T. S. Kuo, S. Das Gupta, Phys. Rev. C 37 (1988) 22532256.

[16] G. M. Welke, M. Prakash, T. T. S. Kuo, S. Das Gupta, C. Gale, Phys.

[17] C. Gale, G. M. Welke, M. Prakash, S. J. Lee, S. Das Gupta, Phys. Rev. C 41 (1990) 1545.

[18] R. B. Wiringa, Phys. Rev. C 38 (1988) 2967.

[19] P. Danielewicz, et al., Phys. Rev. C 38 (1988) 120.

1225 [20] P. Danielewicz, Nucl. Phys. A673 (2000) 375.

[21] P. Danielewicz, Acta Physica Polonica B33 (2002) 45.

[22] P. Danielewicz, R. Lacey, W. G. Lynch, Science 298 (5598) (2002) 15921596.

[23] C. Constantinou, B. Muccioli, M. Prakash, J. M. Lattimer, Phys. Rev. C 1230

[24] G. E. Brown, J. H. Gunn, P. Gould, Nucl. Phys. 46 (1963) 598.

[25] C. Constantinou, B. Muccioli, M. Prakash, J. M. Lattimer, Phys. Rev. C 89 (2014) 065802.

[26] C. Constantinou, B. Muccioli, M. Prakash, J. M. Lattimer, Annals of 1235

[27] J. M. Lattimer, F. D. Swesty, Nucl. Phys. A 535 (1991) 331.

[28] F. D. Swesty, J. M. Lattimer, E. S. Myra, Astrophys. J. 425 (1994) 195. 
[29] H. Shen, H. Toki, K. Oyamatsu, K. Sumiyoshi, Nucl. Phys. A637 (1998) 435.

1240 [30] K. Sumiyoshi, S. Yamada, H. Suzuki, Astrophys. J. 667 (2007) 382-394.

[31] M. Hempel, J. Schaffner-Bielich, Nucl. Phys. A837 (2010) 210-254.

[32] G. Shen, C. J. Horowitz, E. O'Connor, Phys. Rev. C 83 (2011) 065808.

[33] G. Shen, C. J. Horowitz, S. Teige, Phys. Rev. C 83 (3) (2011) 035802.

[34] E. O'Connor, C. D. Ott, Astrophys. J. 730 (2) (2011) 70.

[35] M. Hempel, T. Fischer, J. Schaffner-Bielich, M. Liebendörfer, Astrophys. J. 748 (2012) 70.

[36] A. W. Steiner, M. Hempel, T. Fischer, Astrophys. J. 774 (2013) 17.

[37] M. Carmell, Ph.D. thesis, Stony Brook University, 2005.

[38] J. M. Lattimer, M. Prakash, Astrophys. J. 550 (2001) 426.

[39] J. M. Lattimer, Y. Lim, Astrophys. J. 771 (2013) 51.

[40] S. Weinberg, John Wiley \& Sons, Inc., New York, 1972.

[41] P. Haensel, J. P. Lasota, J. L. Zdunik, Astron. \& Astrophys. 344 (1999) 151.

[42] S. Koranda, N. Stergioulas, J. L. Friedman, Astrophys. J. 488 (1997) 799.

${ }_{1255}$ [43] J. M. Lattimer, M. Prakash, in: S. Lee (Ed.), From Nuclei to Neutron Stars, World Scientific, Singapore, 2011, p. 275.

[44] C. E. Rhoades, R. Ruffini, Phys. Rev. Lett. 32 (1974) 324.

[45] J. M. Lattimer, Ann. Rev. Nucl. Part. Sci. 62 (2012) 488.

[46] S. Gandolfi, J. Carlson, S. Reddy, Phys. Rev. C 85 (2012) 032801.

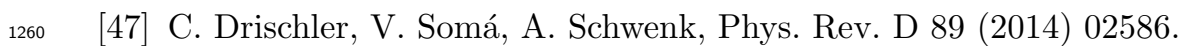


[48] G. Baym, C. J. Pethick, P. Sutherland, Astrophys. J. 170 (1971) 299.

[49] D. Kobyakov, C. Pethick, Phys. Rev. Lett. 112 (2014) 112504.

[50] B. Link, R. I. Epstein, J. M. Lattimer, Phys. Rev. Lett. 83 (1999) 3362.

[51] E. Brown, R. Rutledge, L. Bildsten, Astrophys. J. Lett. 504 (1998) 95.

${ }_{1265}[52]$ D. Page, J. M. Lattimer, M. Prakash, A. W. Steiner, Astrophys. J. Supp. 155 (2004) 623-650.

[53] D. Blaschke, H. Grigorian, D. Voskresensky, Astron. \& Astrophys. 368 (2001) 561.

[54] A. Sedrakian, eprint arXiv:1509.06986.

${ }_{1270}[55]$ K. Hebeler, J. M. Lattimer, C. J. Pethick, A. Schwenk, Astrophys. J. 773 (2013) 11.

[56] E. Lipparini, S. Stringari, Phys. Rev. C 175 (1989) 103.

[57] W. D. Myers, W. J. Swiatecki, Ann. Phys. 55 (1969) 395.

[58] W. D. Myers, W. J. Swiatecki, Nucl. Phys. 81 (1966) 1.

1275 [59] J. M. Lattimer, A. W. Steiner, EPJA 50 (2014) 40.

[60] J. M. Lattimer, in: D. Pines, R. Tamagaki, S. Tsuruta (Eds.), The Structure and Evolution of Neutron Stars, Addison-Wesley, New York, 1992, p. 50 .

[61] J. M. Lattimer, in: A. Ansari, L. Satpathy (Eds.), Nuclear Equation of State, World Scientific, Singapore, 1996, p. 83.

[62] C. F. von Weizsäcker, Zeit. für Phys. 96 (1935) 431.

[63] L.-W. Chen, C. M. Ko, B.-A. li, J. Xu, Phys. Rev. C 82 (2010) 024321.

[64] K. Hebeler, J. M. Lattimer, C. J. Pethick, A. Schwenk, Phys. Rev. Lett. 105 (2010) 161102. 
1285

[66] L. Ray, Phys. Rev. C 19 (1979) 1855.

[67] A. Krasznahorkay, J. A. Balanda, J. A. Bordewijk, M. N. Brandenburg, et al., Nucl. Phys. A 567 (1994) 521.

[68] A. Krasznahorkay, P. Fujiwara, P. van Aarlo, H. Akimune, I. Daito, et al.,

[69] A. Trzeinska, P. Jastrwebski, F. J. Hartmann, R. Schmidt, T. von Egidy, B. Klos, Phys. Rev. Lett. 87 (2001) 082501.

[70] A. Klimkiewicz, N. Paar, P. Adrich, M. Fallot, K. Boretzky, et al., Phys. Rev. C 76 (2007) 051603(R).

[71] S. Terashima, H. Sakaguchi, H. Takeda, T. Ishikawa, M. Itoh, et al., Phys. Rev. C 77 (2008) 024317.

[72] M. Warda, M. Centelles, X. V. nas, X. Roca-Maza, Acta Phys. Pol. B 43 (2012) 209.

[73] P. Danielewicz, J. Lee, Nucl. Phys. A 922 (2014) 1.

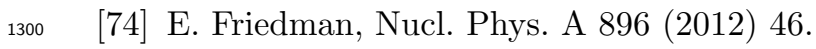

[75] B. C. Clark, L. J. Kerr, S. Hama, Phys. Rev. C 67 (2003) 054605.

[76] J. Zenhiro, et al., Phys. Rev. C 82 (2010) 044611.

[77] V. E. Starodubsky, N. M. Hintz, Phys. Rev. C 49 (1994) 2118.

[78] A. Krasznahorkay, N. Paar, D. V. et al., Physica Scripta T154 (2013) 014018.

[79] A. Tamii, I. Poltoratska, P. von Neumann-Cosel, Y. Fujita, T. Adachi, et al., Phys. Rev. Lett. 107 (2011) 062502.

[80] X. Roca-Maza, M. Brenna, G. Coló, M. Centelles, X. Viñas, et al., Phys. Rev. C 88 (2013) 024316. 
[81] P.-G. Reinhard, W. Nazarewicz, Phys. Rev. C 81 (2010) 051303.

[82] L. Trippa, G. Coló, E. Vigezzi, Phys. Rev. C 77 (2008) 061304.

[83] M. B. Tsang, Y. Zhang, P. Danielewicz, M. Famiano, Z. Li, W. G. Lynch, A. W. Steiner, Phys. Rev. Lett. 102 (12) (2009) 122701.

[84] J. S. Read, B. D. Lackey, B. J. Owen, J. L. Friedman, Phys. Rev. D 79 (2009) 124032.

[85] F. Özel, D. Psaltis, T. Güver, G. Baym, C. Heinke, S. Guillot, eprint http://arxiv.org/abs/1505.05155 arXiv: 1505. 05155.

[86] M. G. Alford, S. Han, eprint arXiv:1508.01261.

[87] J. van Paradijs, Astrophys. J. 234 (1979) 609.

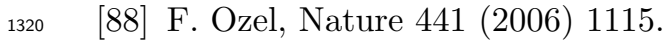

[89] A. W. Steiner, J. M. Lattimer, E. F. Brown, Astrophys. J. 722 (2010) 33.

[90] F. Özel, D. Psaltis, Phys. Rev. D 80 (2009) 103003.

[91] F. Özel, G. Baym, T. Güver, Phys. Rev. D 82 (2010) 101301.

[92] V. Suleimanov, J. Poutanen, M. Revnivtsev, K. Werner, Astrophys. J 742 (2011) 122 .

[93] J. Nättilä, A. W. Steiner, J. J. E. Kajava, V. F. Suleimanov, J. Poutanen, eprint arXiv:1509.06561.

[94] J. van Paradijs, F. Verbunt, R. A. Shafer, K. A. Arnaud, Astron. \& Astrophys. 182 (1987) 47-50.

1330 [95] L. Bildsten, E. E. Salpeter, I. Wasserman, Astrophys. J. 384 (1992) 143.

[96] R. Rutledge, L. Bildsten, E. Brown, G. Pavlov, E. Zavlin, Astrophys. J. $514(1999) 945$.

[97] M. Rajagopal, R. W. Romani, Astrophys. J. 461 (1996) 327. 
[98] V. E. Zavlin, G. G. Pavlov, Y. A. Shibanov, Astron. \& Astrophys. 315

1335

[99] C. O. Heinke, G. B. Rybicki, R. Narayan, J. E. Grindlay, Astrophys. J. $644(2006) 1090$.

[100] N. A. Webb, D. Barret, Astrophys. J. 671 (2007) 727.

[101] S. Guillot, M. Servillat, N. A. Webb, R. E. Rutledge, Astrophys. J. 772 (2013) 7.

[102] A. W. Steiner, J. M. Lattimer, E. F. Brown, Astrophys. J. Lett. 765 (2013) L5.

[103] M. Servillat, C. O. Heinke, W. C. G. Ho, J. E. Grindlay, J. Homg, M. van den Berg, S. Bogdanov, MNRAS 423 (2012) 1556.

${ }_{1345}$ [104] C. O. Heinke, H. N. Cohn, P. M. Lugger, N. A. Webb, W. C. G. Ho, J. Anderson, S. Campana, S. Bogdanov, D. Haggard, A. M. Cool, J. E. Grindlay, MNRAS 444 (2014) 443.

[105] J. M. Lattimer, A. W. Steiner, EPJA submitted (2015).

[106] B. Barker, R. O’Connell, Phys. Rev. D 12 (1975) 329.

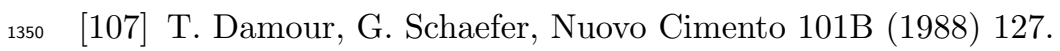

[108] J. M. Lattimer, B. F. Schutz, Astrophys. J. 629 (2005) 979.

[109] K. Gendreau, Z. Arzoumanian, T. Okajima, SPIE 8443 (2012) 13.

[110] M. Feroci, L. Stella, M. van der Klis, et al., Ex. Ast. 34 (2012) 415.

[111] G. G. Pavlov, V. E. Zavlin, Astrophys. J. 490 (1997) 91.

[112] J. Poutanen, M. Gierlinski, MNRAS 343 (2003) 1301.

[113] S. Bogdanov, G. B. Rybicki, J. E. Grindlay, Astrophys. J. 670 (2007) 668.

[114] D. A. Leahy, S. M. Morsink, C. Cadeau, Astrophys. J. 672 (2008) 1119. 
[115] T. E. Strohmayer, W. Zhang, J. H. Swank, Astrophys. J. 487 (1997) 77.

[116] M. C. Miller, F. K. Lamb, Astrophys. J. 499 (2009) 37.

${ }_{1360}$ [117] D. Psaltis, F. Özel, D. Chakrabarty, Astrophys. J. 787 (2014) 136.

[118] M. C. Miller, F. K. Lamb, Astrophys. J. 808 (2015) 31.

[119] S. M. Morsink, D. A. Leahy, C. Cadeau, J. Braga, Astrophys. J. 663 (2007) 1244.

[120] K. Yagi, N. Yunes, Phys. Rev. D 88 (2013) 023009.

${ }_{1365}$ [121] M. Bauböck, E. Berti, D. Psaltis, F. Özel, Astrophys. J. 777 (2013) 68.

[122] J. Abadie, et al., Classical and Quantum Gravity 27 (2010) 173001.

[123] E. E. Flanagan, T. Hinderer, Phys. Rev. D 77 (2008) 021502.

[124] S. Postnikov, M. Prakash, J. M. Lattimer, Phys. Rev. D 82 (2010) 024016.

[125] B. D. Lackey, K. Kyutoku, M. Shibara, P. R. Brady, J. L. Friedman, Phys. Rev. D 85 (2012) 044051.

[126] B. D. Lackey, K. Kyutoku, M. Shibara, P. R. Brady, J. L. Friedman, Phys. Rev. D 89 (2014) 043009.

[127] J. S. Read, B. Luca, J. D. E. Creighton, J. L. Friedman, et al., Phys. Rev. D 88 (2013) 044042.

1375 [128] S. Bernuzzi, T. Dietrich, A. Nagar, Phys. Rev. Lett. 115 (2015) 091101.

[129] A. Bauswein, N. Stergioulas, H.-T. Janka, Phys. Rev. D 90 (2014) 023002.

[130] K. Takami, L. Rezzolla, B. Luca, J. Ph.: Conference Series 600 (2015) 012056.

[131] J. M. Lattimer, M. Prakash, Science 304 (2004) 536.

1380 [132] P. Haensel, J. L. Zdunik, M. Bejger, J. M. Lattimer, Astron. \& Astrophys. 502 (2009) 605. 
[133] S. L. Shapiro, S. A. Teukolsky, I. Wasserman, Astrophys. J. 272 (1983) 702.

[134] S. L. Shapiro, S. A. Teukolsky, I. Wasserman, Nature 340 (1989) 451.

${ }_{1385}$ [135] J. W. T. Hessels, S. M. Ransom, I. H. Stairs, P. C. C. Freire, V. M. Kaspi, F. Camilo, Nature 311 (2006) 1901.

[136] P. Podsiadlowski, J. D. M. Dewi, P. Lesaffre, J. C. Miller, W. G. Newton, J. R. Stone, MNRAS 361 (2005) 1243.

[137] M. Kramer, I. H. Stairs, R. N. Manchester, et al., Science 314 (2006) 97.

${ }_{1390}$ [138] M. Prakash, T. L. Ainsworth, J. P. Blaizot, H. Wolter, in: T. T. S. Kuo, J. Speth (Eds.), Windsurfing the Fermi Sea, Volume II, Elsevier, Amsterdam, 1987, p. 357.

[139] G. Baym, C. Pethick, Landau Fermi-Liquid Theory, Wiley Interscience, New York, 1991.

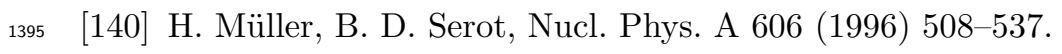

[141] C. B. Das, S. Das Gupta, C. Gale, B.-A. Li, Phys. Rev. C 67 (2003) 034611.

[142] S. Hama, B. C. Clark, E. D. Cooper, H. S. Sherif, R. L. Mercer, Phys. Rev. C 41 (1990) 2737-2755.

1400 [143] P.-G. Reinhard, D. J. Dean, W. Nazarewicz, J. Dobaczewski, J. A. Maruhn, M. R. Strayer, Phys. Rev. C 60 (1999) 014316.

[144] H. T.-. Janka, T. Zweger, R. Monchmeyer, Astron. \& Astrophys. 268 (1993) 360.

[145] A. Bauswein, H. T.-. Janka, R. Oeschin, Phys. Rev. D 82 (2010) 084043.

${ }_{1405}$ [146] K. Hotokezake, K. Kiuchi, K. Kyutoku, T. Muranushi, Y.-i. Sekiguchi, M. Shibata, M. Yanuguchi, Phys. Rev. D 88 (2013) 044026. 
[147] A. Foucart, K. Hotokesake, K. Hotokesake, K. Hotokesake, K. Hotokesake, K. Hotokesake, K. Hotokesake, Phys. Rev. D 90 (2014) 024026.

[148] J. D. Kaplan, C. D. Ott, E. P. O'Connor, K. Kiuchi, L. Roberts, M. Duez, Astrophys. J. 790 (1) (2014) 19.

[149] X. Zhang, M. Prakash, in preparation.

[150] S. Fantoni, B. L. Friman, V. R. Pandharipande, Phys. Lett. B 104 (1981) 89.

[151] J. W. Negele, K. Yazaki, Phys. Rev. Lett. 47 (1981) 71.

1415 [152] J. Blaizot, B. Friman, Nucl. Phys. A 372 (12) (1981) 69.

[153] M. Prakash, J. Wambach, Z. Ma, Phys. Lett. B 128 (1983) 141.

[154] C. Mahaux, P. F. Bortignon, R. A. Broglia, C. H. Dasso, Phys. Rep. 120 (1985) 1.

[155] C. J. Pethick, G. M. Carneiro, Phys. Rev. A 7 (1973) 304.

${ }_{1420}$ [156] S. Fantoni, B. L. Friman, V. R. Pandharipande, Nucl. Phys. A 399 (1983) 51.

[157] P. Danielewicz, G. Odyniec, Phys. Lett. B 157 (1985) 146.

[158] H. A. Gustafsson, H. H. Gutbrod, B. Kolb, H. Löhner, B. Ludewigt, A. M. Poskanzer, T. Renner, H. Riedesel, H. G. Ritter, A. Warwick, F. Weik, H. Wieman, Phys. Rev. Lett. 52 (1984) 1590.

[159] P. Siemens, J. O. Rasmussen, Phys. Rev. Lett. 42 (1979) 880.

[160] J. Y. Ollitrault, Phys. Rev. D 46 (1992) 229.

[161] H. Stöcker, W. Greiner, Phys. Reports. 137 (1986) 277.

[162] M. Jaminon, C. Mahaux, P. Rochus, Nucl. Phys. A365 (1981) 371. 
1430

[163] E. D. Cooper, S. Hama, S. Hama, S. Hama, S. Hama, S. Hama, S. Hama, Phys. Rev. C 47 (1993) 297.

[164] W. Zuo, I. Bombaci, U. Lombardo, EPJA 50 (2) (2014) 1-16.

[165] T. Gaitanos, M. Kaskulov, Nucl. Phys. A 899 (2013) 33.

[166] B.-A. Li, C. B. Das, S. Das Gupta, C. Gale, Phys. Rev. C 88 (2004) 1435

[167] B.-A. Li, C. B. Das, S. Das Gupta, C. Gale, Nucl. Phys. A 735 (2004) 563.

[168] M. Prakash, I. Bombaci, M. Prakash, P. J. Ellis, J. M. Lattimer, Phys. Rep. 280 (1997) 1.

1440

[169] C. O. Heinke, W. C. G. Ho, Astrophys. J. Lett. 719 (2010) L167.

[170] B. Posselt, G. Pavlov, S. V., O. Kargaltsev, Astrophys. J. 779 (2013) 186.

[171] W. Ho, K. G. Elshamouty, C. O. Heinke, A. Y. Potekhin, Phys. Rev. C 91 (2015) 015806.

[172] D. Page, M. Prakash, J. M. Lattimer, A. W. Steiner, Phys. Rev. Lett. 106 1445

[173] P. S. Shternin, D. G. Yakovlev, C. O. Heinke, W. C. G. Ho, D. J. Patnaude, MNRAS 412 (2011) 108. 